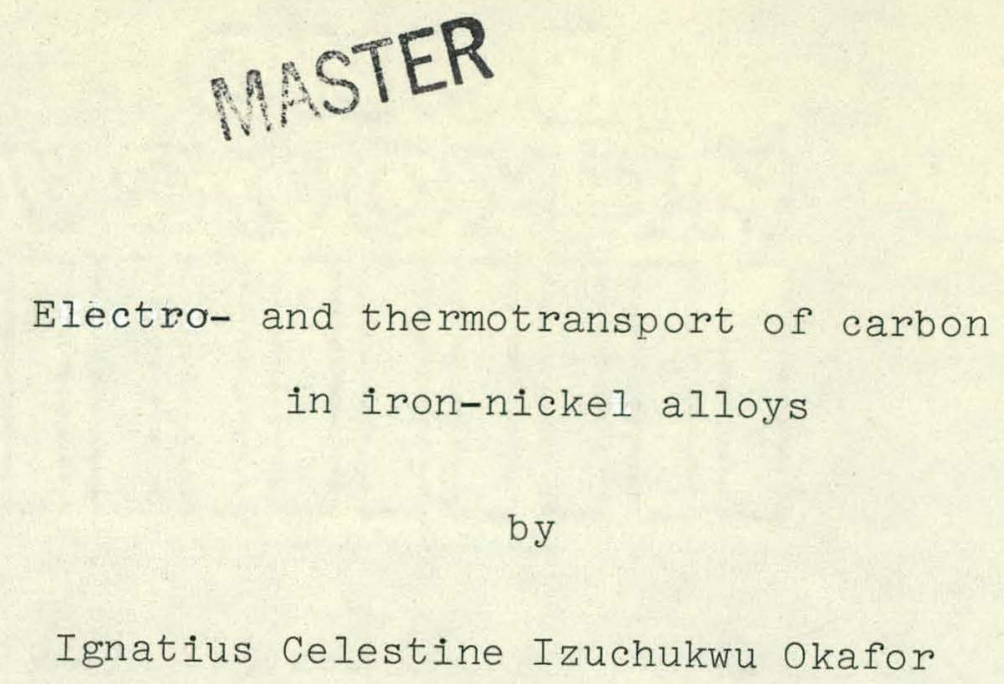

IS-T - 887

PhD Thesis submitted to Iowa State University

Ames Laboratory, DOE

Iowa State University

Ames, Iowa 50011

Date Transmitted: $\quad$ March 1980

PREPARED FOR THE U.S. DEPARTMENT OF ENERGY UNDER CONTRACT NO. W-7405-eng-82 


\section{DISCLAIMER}

This report was prepared as an account of work sponsored by an agency of the United States Government. Neither the United States Government nor any agency Thereof, nor any of their employees, makes any warranty, express or implied, or assumes any legal liability or responsibility for the accuracy, completeness, or usefulness of any information, apparatus, product, or process disclosed, or represents that its use would not infringe privately owned rights. Reference herein to any specific commercial product, process, or service by trade name, trademark, manufacturer, or otherwise does not necessarily constitute or imply its endorsement, recommendation, or favoring by the United States Government or any agency thereof. The views and opinions of authors expressed herein do not necessarily state or reflect those of the United States Government or any agency thereof. 


\section{DISCLAIMER}

Portions of this document may be illegible in electronic image products. Images are produced from the best available original document. 


\section{DISCLAIMER}

This book was prepared as an account of work sponsored by an agency of the United States Government. Neither the United States Government nor any agency thereof, nor any of their employees, makes any warranty, express or implied, or assumes ary legal liability or responsibility for the accuracy, completeness or usefulness of any information, apparatus, product, or process disclosed, or represents that its use would not infringe privately owned rights. Reference herein to any specific commercial product, process, or service by trade name, trademark, manufacturer, or otherwise, does not necessarily constitute or imply its endorsement, recommendation, or favoring by the United States Government or any agency thereof. The views and opinions of authors expressed herein do not necessarily state or reflect those of the United States Government or any agency thereof.

Printed in the United States of America

Available from

National Techrical Irformation Service

U.S. Department of Commerce

5265 Port Royal Road

Springfield, VA 22161 


\title{
Electro- and thermotransport of carbon \\ in iron-nickel alloys
}

by

Ignatius Celestine Izuchukwu Okafor

\author{
A Dissertation Submitted to the \\ Graduate Faculty in Partial Fulfillment of the \\ Requirements for the Degree of \\ DOCTOR OF PHILOSOPHY
}

Department: Materials Science and Engineering

Major: Metallurgy

Approved:

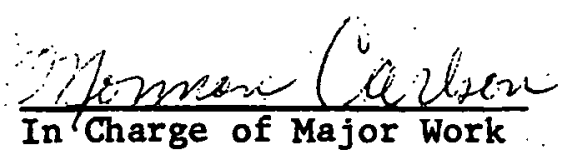

$\frac{\text { DUd the Major Department }}{\text { For the }}$

$\frac{201}{\text { For the Graffate college }}$

Iowa State University

Ames, Iowa 
TABLE OF CONTENTS

Abstract

INTRODUCTION

ELECTROTRANSPORT THEORY

General Concepts

THERMOTRANSPORT THEORY

General Concepts

EXPERIMENTAL PROCEDURE

Equipment

Alloy Preparation

Solubility Studies

Resistivity Studies

Electrotransport Studies

34

Determination of Electrotransport Parameters

35

Thermotransport and Determination of $Q^{*}$

EXPERIMENTAL RESULTS

40

Solubility Studies

40

Resistivity Studies

44

Electrotransport Studies

51

Thermotransport and Determination of $Q^{*}$

DISCUSSION

80

BIBLIOGRAPHY

82

ACKNOWLEDGMENTS

85

APPENDIX A

86

APPENDIX B

93 


\title{
iii
}

\author{
Electro- and thermotransport of carbon \\ in iron-nicke1 alloys
}

Ignatius Celestine Izuchukwu Okafor

Under the supervision of 0 . N. Carlson

From the Department of Matcrials Science and Engineering Iowa State University

Results of studies on the electrotransport, thermotransport and chemical diffusion of carbon in $\gamma$-phase iron -32.5 wt \% nickel alloys are described. The transport parameters, $\mathrm{Z}^{*}, \mathrm{Q}^{*}$ and $\mathrm{D}$ were measured as a function of temperature and carbon concentration and values for the activation energies for diffusion and electrotransport were obtained.

The solubility 1 imit of carbon in $\gamma-\mathrm{Fe}-32.5$ wt $\% \mathrm{Ni}$ for the temperature range 850 to $1200^{\circ} \mathrm{C}$ and the electrical resistivity versus temperature for carbon concentrations of $0.0,0.1,0.2,0.5$ and 0.86 wt $\% \mathrm{C}$ between 25 and $1350^{\circ} \mathrm{C}$ were determined. An anomalously large mass transport effect observed in two-phase alloy during thermotransport experiments was investigated for the Fe-Ni-C system.

DOE Report IS-T-887*. This work was performed under Contract W-7405-eng-82 with the Department of Energy. 


\section{INTRODUCTION}

Iron-base alloys with variations in the chromium and nicke1

concentrations form the basis of a number of austenitic stainless steels and other speciality iron alloys such as Incoloy and Hastelloy. The alloys have extensive high temperature applications in turbines, steam generators and piping in electric power generating plants; in gas cracking and other equipment in the petroleum refining industry; and in sodium-cooled fast breeder nuclear reactors for the fuel cladding, heat exchangers, piping, valves and various structural components. Although the environmental conditions in these applications are strikingly different, the materials for the most part change in microstructure and composition particularly in the nonmetallic elements such as carbon, nitrogen and boron after long-term service at high temperatures (1). Numerous investigators (2-11) have studied the relationship between the composition, microstructure and mechanical properties of these materials. Extensive work has also been done on the importance of the carbon and nitrogen contents of these steels on the mechanical properties (6-10) and the microstructural stability (12-15).

The major motivation for this work is the lack of adequate knowledge of interst1t1al solute transport in the high nickel-1ron base alloys as indicated in the Bethe Report (16) on the LMFBR Alloy Development Program. The report evaluated numerous commercial alloys based on their mechanical properties. Quite a few alloys met these general requirements, but more specific properties such as neutronics, 
radionuclide transport, swelling, irradiation creep, thermal creep, post irradiation ductility, and fuel and sodium compatibility were satisfied by only a few alloys. High-nickel (15-60\% Ni) austenitic steels fared much better than their low-nickel counterparts which exhibit more than $5 \%$ swelling at $3 \times 10^{23} \mathrm{n} / \mathrm{cm}^{2} \mathrm{flux}$. Vanadium-base alloys have excessive corrosion at normal sodium oxygen levels. Ferritic steels fail only at high cladding temperatures thus giving room for further development. Nickel-base alloys exhibit poor neutronics; colbalt-base, niobium-base and molybdenum-base alloys all have their respective limitations (16) (Table 1).

Among the high-nickel austenitic steels (15-60\% Ni), alloys containing from $30-60 \%$ nickel exhibit minimum amount of swelling upon neutron irradiation (Fig. 1). Incoloy 800 , a commercial steel with composition 32.5 wt $\% \mathrm{Ni}, 21$ wt $\% \mathrm{Cr}, 0.05$ wt $\% \mathrm{C}, 0.4$ wt $\% \mathrm{Ti}$, 0.4 wt $\% \mathrm{Al}, 0.75$ wt $\% \mathrm{Mn}, 0.5$ wt $\% \mathrm{Si}$ and 0.4 wt $\% \mathrm{Cu}$ - the rest being iron - belongs to this group of alloys. The problem of interstitial migration in these candidate materials has been of interest to different investigators $(17,18)$.

The aim of this work is to study the transport of carbon in a model steel that is austenitic and contains an amount of nickel (32.5 wt \%) that would provide minimal swelling under a flux of neutrons. The alloy of interest, $\mathrm{Fe}-32.5$ wt $\% \mathrm{Ni}$, constitutes the major part of Incoloy 800 . In this study the transport of the solvent substitutional atoms ( $\mathrm{Fe}$ and $\mathrm{Ni}$ ) is assumed to be negligible relative to the fast 
Table 1. Subjective alloy evaluation ${ }^{a, b}$

\begin{tabular}{|c|c|c|c|c|c|c|c|c|}
\hline $\begin{array}{l}\text { Alloy system } \\
\text { property }\end{array}$ & $\begin{array}{c}\text { Low-nickel } \\
\text { austenitic } \\
\text { steels } \\
(<15 \% \mathrm{Ni})\end{array}$ & $\begin{array}{l}\text { Vanadium- } \\
\text { base }\end{array}$ & $\begin{array}{l}\text { High-nickel } \\
\text { austenitic } \\
\text { steels } \\
(15-60 \% \mathrm{Ni})\end{array}$ & $\begin{array}{l}\text { Ferritic } \\
\text { steels }\end{array}$ & $\begin{array}{c}\text { Nicke1- } \\
\text { base } \\
(>60 \% \mathrm{Ni})\end{array}$ & $\begin{array}{l}\text { Cobalt- } \\
\text { base }\end{array}$ & $\begin{array}{l}\text { Niobium- } \\
\text { base. }\end{array}$ & $\begin{array}{c}\text { Molybdenum- } \\
\text { base }\end{array}$ \\
\hline Neutronics & $* *$ & $* * * * *$ & $*$ & $* x \cdot x$ & $F^{(4)}$ & $F^{(5)}$ & $\mathrm{F}$ & $F$ \\
\hline $\begin{array}{l}\text { Sodium } \\
\text { compatibility }\end{array}$ & $* *$ & $\mathrm{~F}^{(2)}$ & $*$ & $* * * * *$ & & $* * *$ & F & $* \star * * *$ \\
\hline $\begin{array}{l}\text { Radionuclide } \\
\text { transport }\end{array}$ & * & $* * *$ & $* *$ & $*$ & $* *$ & & & $* * * * *$ \\
\hline Swelling & $F^{(I)}$ & $* *$ & $x+x$ & $* * * *$ & $* * * * x$ & $* * *$ & * & $* *$ \\
\hline $\begin{array}{l}\text { Fuel } \\
\text { compatibility }\end{array}$ & $* x *$ & $*$ & $x+x$ & $* x *$ & $* *$ & $* *$ & $*$ & $* * * * x$ \\
\hline $\begin{array}{l}\text { Irradiation } \\
\text { creep }\end{array}$ & & $* *$ & $* * x$ & $* * *$ & $* x+x$ & $* * *$ & * & $* *$ \\
\hline Thermal creep & * & * & $* * *$ & $F^{(3)}$ & $* * * *$ & $* * * *$ & * & $* * *$ \\
\hline Ductility & $* *$ & * & * & 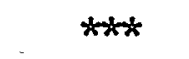 & * & * & $* x *$ & \\
\hline
\end{tabular}

${ }^{a}$ Asterisks indicate satisfaction, the more the asterisks, the better the property is satisfied.

$b_{F}=$ Fails to satisfy requirements; $1=$ Greater than $5 \%$ swelling at $3 \times 10^{23} \mathrm{n} / \mathrm{cm}^{2}$ neutron flux; 2 = Excessive corrosion at normal sodium oxygen levels; warrants development; $3=$ Fails only at high cladding temperatures; warrants development; $4=$ Can remain as candidate control pin cladding; 5 = Possibly acceptable in harder spectrum of carbide/nitride core. 
COMPOSITION DEPENDENCE OF SWELLING (GE)

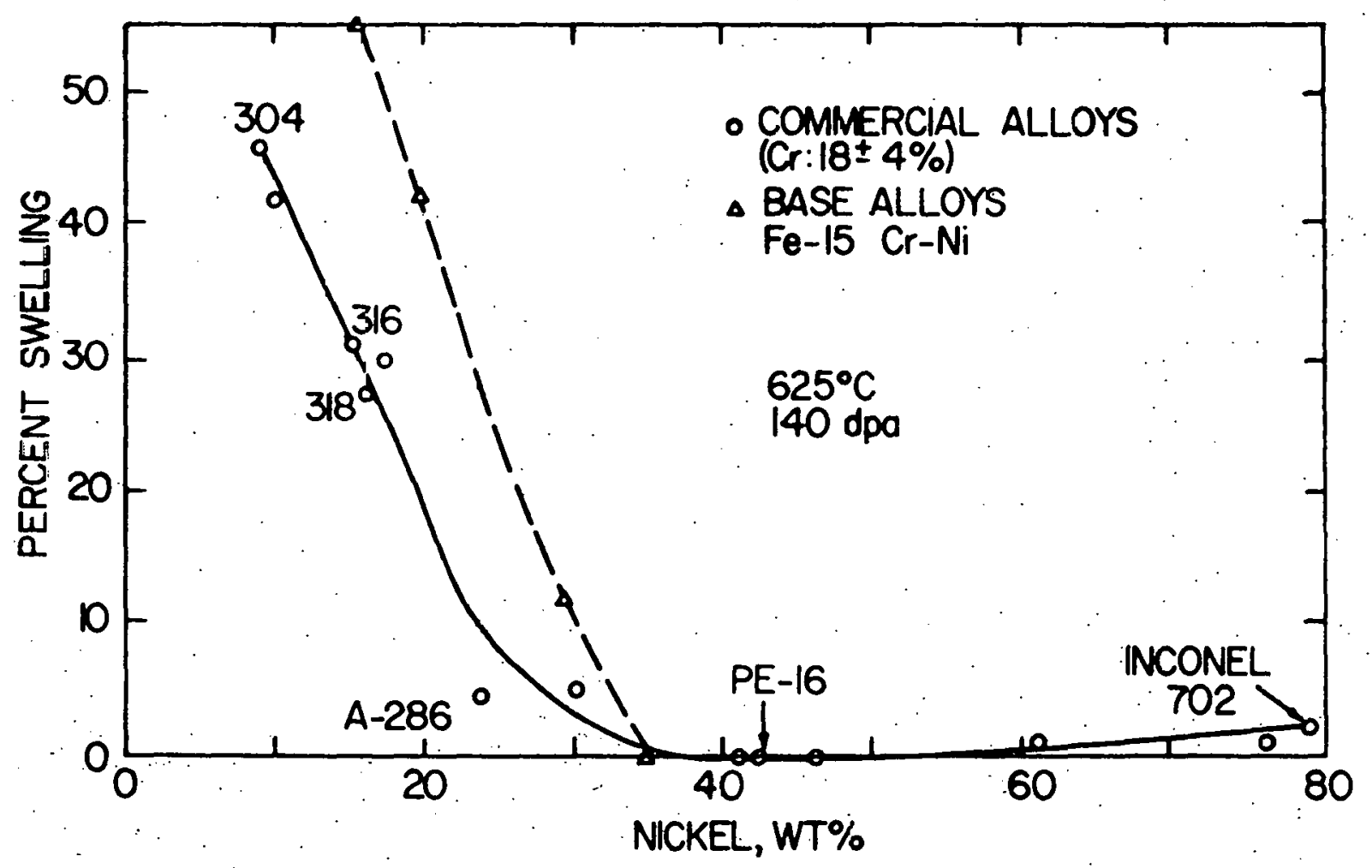

Figure 1. Composition dependence of swelling for nickel content of commercial alloy steels. 
diffusing carbon interstitials. Perhaps it would be more applicable to study the carbon migration in the commercial Incoloy 800 , but the effects of the other nine alloying components of this steel, including silicon, on carbon mobility would make the study considerably more difficult. 
ELECTROTRANSPORT. THEORY

\section{General Concepts}

When a direct current is passed through a pure metal or an alloy, the charge carrier (electrons) possess considerable momentum which may be transferred to the metal (or alloy) ions.

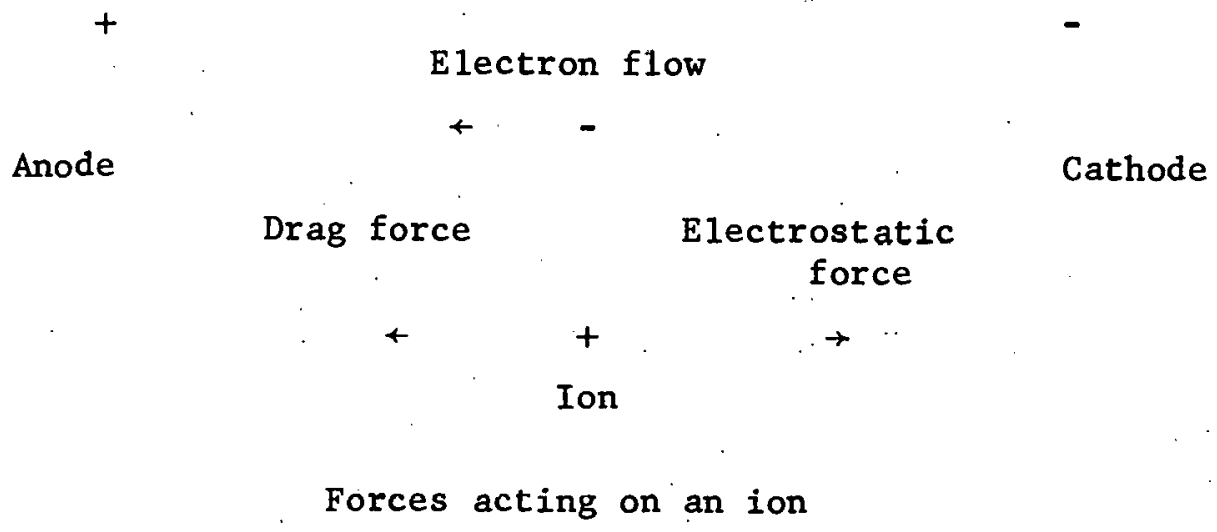

The ions therefore experience two forms of force; namely, the electrostatic force exerted by the applied electric field and secondly, the "drag force" resulting from momentum exchange with the electrons. Assuming that the metal atoms are positively charged due to the loss of electrons to the "electron gag" (19); the applied electric field $\bar{E}$, acts on an ion $i$ with a force

$$
\mathbf{F}=\mathrm{eEZ}_{\mathbf{i}}
$$

where $e$ is the electronic charge and $z_{i}$ the actual valence of the ion. The interaction of the conduction electrons with the ion results in an 
additional force, $\mathrm{F}_{e i}$, so that the total force on the ion is

$$
F_{i}=e E Z_{i}-F_{e i}
$$

If we assume that the drag force, $F_{e i}$, of the electrons on component $i$ is proportional to the electric field, we may write

$$
F_{\text {ei }}=\delta_{e i} E
$$

where $\delta_{e i}$ is the drag coefficient. The resultant net force is then

$$
\begin{aligned}
F_{i} & =e E\left(Z_{i}-\frac{\delta_{e i}}{e}\right) \\
& =e E z_{i}^{*}
\end{aligned}
$$

where $z_{i}^{*}$ is the "effective" valence of the ion. The direction of the resultant force on ion $i$ is, therefore, determined by the sign of $z_{i}^{*}$, i.e. by the relative values of $z_{i}$ and $\frac{\delta_{e i}}{e}$. If

(a) $z_{i}>\frac{\delta_{e i}}{e}$, the electrostatic force is greater, then the resultant force is cathode directed and $z_{i}^{*}$ is positive,

(b) $z_{i}<\frac{\delta_{e i}}{e}$, the electron-drag force predominates, the resultant force is anode directed, thus $z_{i}^{*}$ is negative. The ion will migrate with a terminal velocity $v_{i}$, given by

$$
v_{i}=F_{i} B_{i}
$$

where $\mathrm{B}_{i}$ is the absolute mobility. Einstein showed that at temperature T the absolute mobility is related to the diffusion coefficient, $D_{i}$, 
for a completely random process by the relation

$$
B_{i}=\frac{D_{i}}{K T}
$$

where $\mathrm{K}$ is the Boltzman constant. However, the diffusion process in crystalline solids is not completely random, so Eq. 7 is modified to

$$
B_{i}=\frac{D_{i}}{f K T}
$$

where $f$ is a correlation coefficient. For an interstitial, however, $\mathrm{f}=1$. Thus

$$
v_{i}=D_{i} \frac{e E}{K T} z_{i}^{*}
$$

Electric mobility $U_{i}$ is defined as the velocity of the ion per unit electric field. Hence from Eq. 9 the electric mobility becomes

$$
\mathrm{U}_{i}=\frac{\mathrm{v}_{i}}{\mathrm{E}}=\frac{\mathrm{D}_{i} \mathrm{e} \mathrm{Z}_{i}^{*}}{\mathrm{KT}} .
$$

In principle, the electric mobllity, $U_{i}$, can be obtained from electrotransport measurements and $D_{i}$ from diffusion studies, so $z_{i}^{*}$ can be determined. The true valence of the ion, $Z_{i}$, may then be obrained if the drag coefficient, $\delta_{e i}$, is independently determined or vice versa. Within the last twenty-five years or so, a number of attempts have been made to quantitatively describe the electrotransport process in metals and derive expressions for the drag force and the net force (20). The numerous treatments can be grouped into three categories; namely, 
(a) phenomenological treatment using the methods of irreversible thermodynamics,

(b) the semi-empirical methods and

(c) the quantum-mechanical approach in which the electron-ion interactions are closely examined.

In order to indicate the relationships which exist between mass transport and electron transport, two of the most recent approaches for determining the drag force, which fall into category (c), are discussed. Although essentially different, the two models yield virtually the same results, thus permitting one to relate the drag force to a scattering cross-section or a resistivity increment due to the scattering centers.

The dynamical model

Huntington et a1. $(21,22)$ and Fiks $(23,24)$ have presented similar treatments for determining the drag force acting on scattering centers in a current carrying metal. The physical mechanism depends on momentum transfer from the current carriers to the scattering centers. Huntington and Grone (21) showed that the rate of scattering is sufficiently high to justify approximating the effect as a continuous force. Thus the total momentum transferred per unit time to each center is $\mathrm{F}_{\mathrm{ei}}$ given by

$$
F_{e i}=\frac{1}{N_{i}\left(4 \pi^{3}\right)^{2}} \frac{\int}{K} \frac{\int}{K}, \sum_{b} \rho_{\overline{K K}}, b f(\bar{K})[1-f(\bar{K})] W_{\overline{K K}}{ }^{-1} b d^{3} K d^{3} K^{\prime} \quad E q \cdot 11
$$


where $\mathrm{N}_{i}$ is the density of scattering centers, $\mathrm{P}_{\overline{\mathrm{K}}} \overline{\mathrm{K}}^{\mathrm{b}}$ is the momentum transferred to the scattering center by an electron scattered from a state $\bar{K}$ to $\bar{K}^{\prime}(+2 \pi \bar{b}), \bar{b}$ is a reciprocal lattice vector, $f(\bar{K})$ is the electron distribution function and $\mathrm{W}_{\mathrm{KK}} \mathrm{b}$ is the transition probability per second per unit volume. This complicated expression is very much simplified if the Fermi surface is closed and Umklapp process neglected. In the relaxation time approximation it reduces to

$$
F_{e i}=\frac{m^{*}}{N_{i}^{e} \cdot t_{i}} j
$$

where $j$ is the current density, $t_{i}$ is the mean time between collisions $\left(=\frac{\ell_{e i}}{v_{e_{*}}}\right.$; where $\ell_{e i}$ is the mean free path and $v_{e}$ is the electron velocity) and $\mathrm{m}^{*}$ is the effective electron mass. Thus the sign of the drag force depends on the sign of $\mathrm{m}^{*}$, hence on the curvature of the Fermi surface. If we define the electron energy as $\epsilon$; then if $\frac{\partial^{2} \varepsilon}{\partial \mathrm{K}^{2}}>0$, the force is in the samedirection as the electron flow, anode directed. For $\frac{\partial^{2} \epsilon}{\partial K^{2}}<0$, the force is cathode directed and the electrons act 1ike holes. Fiks treatment Fiks treated $\mathrm{F}_{\mathrm{ei}}$ of $\mathrm{Eq} .12$ as a function of the ion scattering cross-section $\sigma$. For electrons and holes the drag force becomes

$$
F_{e i}=n_{e} e_{e i} \sigma_{e i} e E
$$

and

$$
F_{e i}=-\pi_{h} l_{h i} \sigma_{h i} e^{E}
$$


where $n_{e}$ and $n_{h}$ are the electrons and holes concentrations per unit volume respectively. Using Eq. 13a the resultant force becomes

$$
F_{i}=e E\left(z_{i}-n_{e}^{l} e_{i} \sigma_{e i}\right) \cdot \quad E q \cdot 14
$$

Taking the scattering center as an impurity ion, Eq. 14 becomes non-zero. However, for a pure metal with ions in a non-activated state (an activated ion is considered to be a free ion, completely decoupled from the lattice), $z_{i}=n_{e} e_{e i} \sigma_{e i}$, so the force given by Eq. 14 is actually zero. For self-transport Fiks (24) suggested that migration occurs through the movement of activated ions. In the activated state, the ion is considered to have a charge $z_{i}^{0}$, and a scattering crosssection $\sigma_{e i}{ }^{\circ}$, which differ from the corresponding values in the non-activated state. Thus Eq. 14 becomes

$$
F_{i}=e E\left(z_{i}^{0}-z \frac{\sigma_{e i}}{\sigma}\right. \text {. }
$$

where $\mathrm{z}$ and $\sigma$ are the mean values of the system, and $\mathrm{z}=\mathrm{n}_{\mathrm{e}}{ }^{l \sigma}$.

Huntington's treatment Employing Ohms law, Huntington recast

Eq. 12 as

$$
F_{e i}=\operatorname{eEZ} \frac{N_{0}}{N_{i}} \cdot \frac{\rho_{i}}{\rho_{0}} \frac{m^{*}}{\left|m^{*}\right|}
$$

where $\rho_{i}$ is the contribution to the resistivity by the scattering centers, $\rho_{0}$ is the resistivity of the metal and the electron density $\left(n_{e}\right)$ has been replaced by $\mathrm{N}_{0} \mathrm{Z}$. The factor $\frac{\mathrm{m}^{*}}{\left|\mathrm{~m}^{*}\right|}$ simply takes into account the fact that the sign of the current carriers determines the direction of the drag force. Huntington et al. $(21,22)$ used the above equation as a 
basis for analyzing the motion of defects (activated ions) in conductors. They considered the drag force to be a function of position, being a maximum when the ion is at the saddle point half-way from an equilibrium lattice site on its way to fill a vacancy and a minimum at the lattice site. By assuming a sinusoidal variation with the periodicity of the lattice, Huntington and Grone (21) showed that the average net force on an ion undergoing self-transport is

$$
F_{i}=\operatorname{eEZ}\left(1-\frac{1}{2} \cdot \frac{N_{0}}{N_{i}{ }^{2}} \cdot \frac{\rho_{i}^{Z}}{\rho_{0}} \cdot \frac{m^{*}}{\mid m^{*}}\right)
$$

where superscript $\mathrm{Z}$ means that the quantity relates to the saddle point. If the defect is an interstitial ion, the average net force per interstitial is

$$
F_{i}=e E\left(z_{i}-\frac{1}{2} z \frac{N_{0}}{N_{i} z_{i}} \cdot \frac{\rho_{i}^{z_{i}}}{\rho_{0}} \cdot \frac{m^{*}}{m^{*} \mid}\right) \text {. }
$$

So far, the determination of the net force acting on an ion undergoing electrotransport has assumed that the electrostatic component is due to the interaction of the electric field with the unscreened 10n. In the second model we are exposed to the effect of screening the transport ions by the current carriers.

The polarization mode1

Bosvieux and Friedel (25) determined the total force acting on interstitial atoms and matrix atoms in metals based on calculations of 
the perturbation of electron wave functions due to an applied electric field. They considered both the direct polarization effects and the effects arising from the displacement of the Fermi surface due to the passage of an electric current. From an analysis based on a free electron model and Born's first approximation scattering theory, they showed that all ions at lattice sites, including substitutional ions, experience a direct force from the electric field, whereas interstitial ions do not because of a complete screening effect by the charge carriers. The displacement of the Fermi surface causes a polarization which in turn gives rise to a force both parallel and proportional to the applied electric field. The effect of the displacement of the Fermi surface can be explained as follows: if the current carriers are electrons, the displacement of the Fermi surface, given by $\bar{a}=e \bar{E} t$ in $\overline{\mathrm{K}}$-space where $t$ is the mean time of scattering, is equivalent to an increase in the number of electrons traveling towards the anode. These electrons are retarded at the anode side of the impurity and accelerated on the cathode side. Thus, the electron density is increased on the anode side relative to the cathode side which results in an anode directed "indirect polarization force". For a "hole" conductor, the effect is reversed.

For an interstitial ion in an electronic conductor, Bosvieux and Friedel showed that the resultant net force may be written in terms of the resistivity of the impurity in the matrix. They found

$$
F_{i}=-\operatorname{eEZ} \frac{N_{0}}{N_{i}} \cdot \frac{\rho_{i}}{\rho_{0}}
$$


using the previous notation. This is the same result as that obtained in the previous section for the case of a totally screened interstitial ion. The force is in the same direction as the motion of the current carriers (25) and is independent of the valence of the ion. During the jump between two neighboring interstitial sites, the screening charge is considered to be tightly bound and so moves. with the lon. Thus the force given by Eq. 19 above remains constant throughout the jump and represents the average net force acting on an interstitial ion undergoing electrotransport. The calculation for the average net force in self-transport and substitutional transport is more complex. During the jump from a lattice site to a vacant site, the force acting on the ion will vary with position.

In general the two models outlined above are essentially the same in that they relate the forces on the ions to the resistivity of the solvent and the excess resistivity of the migrating ion in the stable and saddle positions. The factor $\rho_{i} \frac{N_{0}}{N_{i}}$ is almost temperature independent, so when the drag force predominates both models predict a decrease in the effective valence with increasing temperature as $\rho^{-1}$. The velocity of the migrating ion is given by Eq. 9 which by using Ohms law becomes

$$
v_{i}=j \frac{D_{i} e \rho_{o} z_{i}^{*}}{K T}
$$

Thio cquation indicates that at a given temperature, the velocity of transport is proportional to the current density. Also, since $z_{i}^{*}$ usually decreases linearly with increasing temperature and $\frac{\rho_{0}}{T}$ is 
temperature independent (as $\rho_{0} \alpha \mathrm{T}$ for metals), the temperature variation of $\frac{v_{i}}{j}$ should be slightly less than that of the diffusion coefficient.

The two models have two main differences anyway.

(a) The dynamical model assumes that the only force acting on the ion at the stable point is the electrostatic force, whereas in the polarization model the electrostatic force is augmented by a force arising from the modification of the electronic charge distribution by the neighboring vacant site.

(b) In the dynamical model no account is taken of screening at the saddle point. The screening reduces the electrostatic force to zero in the polarization model.

There have been several attempts to assess the relative merits of the two models. Experimental measurements of electrotransport have permitted determinations of $\rho$ which are compared to estimates from resistivity measurements. Although the comparisons are inconclusive, there is some evidence to suggest that the best agreement is with the Bosvieux-Friedel polarization model.

Considering the Arrhenius relationship for the temperature dependence of the diffusion coefficient $D=D_{0} \cdot \exp \left(\frac{-Q}{R T}\right), E q \cdot 20$ can be rewritten as

$$
v_{i}=j \frac{D_{0} e^{\frac{-Q}{R T}} e \rho_{0} z^{*}}{K T}
$$




$$
\ln \frac{v_{i}^{T}}{j}=\frac{-Q}{R T}+\ln \left(\frac{D_{0} e \rho_{0} Z^{*}}{K}\right)
$$

where $D_{0}$ is the frequency factor and $Q$ the activation energy.

Nakajima and Hirano (26) reported that the effective charge $z^{*}$ of carbon in $\alpha$-iron is related to the resistivity as

$$
z^{*}=3110 \frac{\Delta \rho_{d}}{\rho_{o}}
$$

where $\Delta \rho_{\mathrm{d}}$ is the resistivity increment due to solution of carbon in $\alpha$-iron.

In our alloy system ( $\gamma$-phase), $\mathrm{z}^{*}$ should have a similar relationship but with a different numerical constant. Taking $z^{*}=\operatorname{constant} \frac{\Delta \rho_{d}}{\rho_{o}}$ where $\Delta \rho_{\mathrm{d}}$ represents the resistivity increment due to solution of carbon in the Fe-Ni $\gamma$-phase alloy, Eq. 22 can be written as

$$
\ln \frac{v_{i}^{T}}{j}=\frac{-Q}{K T}+\ln \left(\frac{\text { const. e } \Delta \rho_{d} D_{o}}{K}\right)
$$

For $D_{o}, \Delta \rho_{d}$ and $Q$ independent of temperature, a $p$ lot of $\ln \frac{v_{i} T}{j}$ versus $\frac{1}{T}$ should be a straight line, and the slope yield the activation energy $Q$ comparable to that obtained from diffusion experiments. 


\section{THERMOTRANSPORT THEORY}

Thermotransport, also known as thermomigration or thermodiffusion, is defined as the migration of matter under the influence of a temperature gradient or the segregation of the constituents of an alloy under the same influence.

The theoretical aspects of thermomigration have remained obscure for a rather long time. It is the thermodynamics of irreversible processes that has furnished a useful formalism for the understanding of this type of phenomenon and has emphasized the link with the inverse phenomenon, the Dufour effect (arising from a heat flux in a system where a concentration gradient prevails). The narrow resemblance between thermotransport and heat conduction appears in the theoretical works of Fiks for which thermodiffusion results from the forces exerted on the atoms by two types of heat carrying agents in a metal: phonons and electrons. The former make intrinsic contributions while the latter account for the electronic contributions.

\section{General Concepts}

Although a few authors $(27,28)$ have discussed a number of approaches to the thermomigration theory, we shall discuss this phenomenon of solute redistribution under a temperature gradient with the ald of irreversible thermodynamics. This method has been used by a few theorists $(29,30,31)$. Detailed proofs of equations can be found in the articles quoted and in standard works of irreversible 
thermodynamics $(30,32)$.

Denbigh's treatment, based on the works of Onsager (29) and de Groot (32) has shown that thermomigration may be described in terms of the following set of phenomenological equations

$$
\begin{array}{lll}
J_{1}=M_{11} X_{1}+M_{12} X_{2}+M_{1 H} X_{H} & \text { Eq. 25a } \\
J_{2}=M_{21} X_{1}+M_{22} X_{2}+M_{2 H} X_{H} & \text { Eq. 25b } \\
J_{H}=M_{H 1} X_{1}+M_{H 2} X_{2}+M_{H H} X_{H} & \text { Eq. 25c }
\end{array}
$$

where $J_{i}=f l u x$ of component $i, J_{H}=$ thermal energy flux, $x_{i}=$ material driving force due to component $i$, and $x_{H}=$ thermal driving force. $M_{11}, M_{22}$ and $M_{H H}$ are related to the component diffusivities and thermal conductivity respectively. The other $M$ coefficients are cross coefficients representing couplings among the heat and material fluxes. These couplings between fluxes explain the Soret $\left(\mathrm{M}_{12}\right)$ and Dufour $\left(\mathrm{M}_{21}\right)$ effects in particular.

For the case of thermal diffusion of an interstitial solute through a stationary solvent lattice, where $J_{2}$ is equal to zero, we would obtain, for dilute solid solutions in general

$$
\begin{aligned}
& J_{2}=0=M_{21} X_{1}+M_{22} X_{2}+M_{2 H} X_{H} \\
& -M_{22} X_{2}=M_{21} X_{1}+M_{2 H} X_{H}
\end{aligned}
$$


Multiply Eq. 27 by $\frac{-\frac{M}{12}}{M_{22}}$ and substituting in Eq. 25 a above we have

$$
J_{1}=\left(M_{11}-\frac{M_{12} M_{21}}{M_{22}}\right) x_{1}+\left(M_{1 H}-\frac{M_{12} M_{2 H}}{M_{22}}\right) x_{H} . \quad \text { Eq: } 28
$$

Similarly, multiplying Eq. 27 by $\frac{-M_{12}}{M_{22}}$ and substituting in Eq. $25 c$,

$$
J_{H}=\left(M_{H 1}-\frac{M_{H 2} M_{21}}{M_{22}}\right) x_{1}+\left(M_{H H}-\frac{M_{H 2} M_{2 H}}{M_{22}}\right) x_{H} \text {. }
$$

Hence we write a set of phenomenological relations for $J_{1}$ and $J_{H}$ in terms of $x_{1}$ and $x_{H}$ putting

$$
M_{11}^{\prime}=\left(M_{11}-\frac{M_{12} M_{21}}{M_{22}}\right), M_{1 H}^{\prime}=\left(M_{1 H}-\frac{M_{12} M_{2 H}}{M_{22}}\right), M_{H 1}^{\prime}=\left(M_{H 1}-\frac{M_{H 2} M_{21}}{M_{22}}\right)
$$

and $M_{H H}^{\prime}=\left(M_{H H}-\frac{M_{H 2} M_{2 H}}{M_{22}}\right)$.

$$
\begin{aligned}
& J_{1}=M_{11}^{\prime} x_{1}+M_{1 H}^{\prime} x_{H}^{\prime} \\
& J_{H}=M_{H 1}^{\prime} x_{1}+M_{H H}^{\prime} x_{H} .
\end{aligned}
$$

Since from the Onsager reciprocity principle, $M_{12}=M_{21}, M_{1 H}=M_{H 1}$ and $M_{H 2}=M_{2 H}$, we see that $M_{H 1}^{\prime}=M_{1 H}^{\prime}$, so that Eqs. 26 and 27 comply with this principle. Thus Eqs. 30 and 31 are equally valid sets of relations for describing the fluxes $J_{1}$ and $J_{H}$ as are Eqs. 25a and 25c. Further, de Groot (32) and Denbigh (33) have each shown that if $J_{1}$ and $J_{H}$ are defined in terms of moles $/ \mathrm{cm}^{2}-\mathrm{sec}$ and $\mathrm{cal} / \mathrm{cm}^{2}-\mathrm{sec}$, respectively, the driving forces, $X_{(1 \text { or } H)}$ will have the form 


$$
\begin{gathered}
\mathrm{X}_{1}=-\mathrm{T} \operatorname{grad}\left(\frac{\mu}{\mathrm{T}}\right)=-\left[\operatorname{grad} \mu-\left(\frac{\mu}{\mathrm{T}}\right) \operatorname{grad} \mathrm{T}\right] \\
\mathrm{X}_{\mathrm{H}}=-\frac{(\operatorname{grad} \mathrm{T})}{\mathrm{T}}
\end{gathered}
$$

where $\mu$ is the chemical potential of the solute at temperature $T$. If the following constraints apply (31) to an interstitial system like the one investigated in the present study

(a) there is only one solid phase $(\gamma)$ in the system and

(b) the temperature gradient exists only along an axis $\mathrm{X}$, then the flux of solute is given by

$$
J_{1}=-M_{11}^{\prime}\left[\left(\frac{d \mu}{d x}\right)-\left(\frac{\mu}{T}\right)\left(\frac{d T}{d x}\right)\right]+\left(\frac{M_{1 H}^{\prime}}{T}\right)\left(\frac{d T}{d x}\right)=0 \text {. Eq. } 34
$$

Furthermore, de Groot (30) and Denbigh (33) have shown that

$$
\left(\frac{\mathrm{J}_{\mathrm{H}}}{\left.\mathrm{J}_{1}\right)_{\mathrm{T}=0}}=Q=\frac{\mathrm{M}_{1 \mathrm{H}}^{\prime}}{\mathrm{M}_{11}^{\prime}}\right.
$$

where $Q$ is the thermal energy transported by one mole of solute in isothermal diffusion (i.e. when $\mathrm{X}_{\mathrm{H}}=0$ ). Then dividing Eq. 34 by $\mathrm{M}_{11}{ }^{\prime}$ and substituting for $Q$ from Eq. 35 into Eq. 34 we obtain

$$
\frac{d \mu}{d x}-\left(\frac{\mu}{T}\right)\left(\frac{d T}{d x}\right)+\left(\frac{Q}{T}\right)\left(\frac{d T}{d x}\right)=0
$$

Using the thermodynamic identity $\mu=\bar{H}-T \bar{S}$ where $\bar{H}$ and $\bar{S}$ are the partial molar enthalpy and entropy of the solute and defining the heat of transport $(29,30) Q^{*}$ by $Q^{*}=Q-\vec{H}$ and substituting into Eq. 36 
we obtain

$$
\left(\frac{Q^{*}}{T}\right)\left(\frac{d T}{d x}\right)+\left(\frac{d \mu}{d x}\right)+\bar{S}\left(\frac{d T}{d x}\right)=0
$$

which gives us the relation between the gradients of temperature and chemical potential at the steady state, $J_{1}=0$. Simplifying we get

$$
-Q^{*}=T\left(\frac{d \mu}{d T}\right)+T \bar{S}
$$

It is necessary to indicate here that $\frac{\mathrm{d} \mu}{\mathrm{dT}}$ in the above equation does not equal $-\overline{\mathrm{S}}$; in contrast to many classical systems, since the constraint on this equality is that of constant composition. Obviously if the steady-state solute concentration were constant with temperature, there would be no thermal diffusion and $Q^{*}$ would be zero.

$Q^{*}$ cannot, however, be calculated a priori from reversibly measured thermodynamic properties. Since $\mu$ and $\overline{\mathrm{S}}$ in Eq. 38 are functions of both the temperature and solute concentration at equilibrium, they can only be evaluated from the knowledge of the steady-state solute distribution.

To make Eq. 38 more tractable, Denbigh makes the assumption that the solution of the solute in the solvent lattice is ideal. So we have

$$
\mu=\mu_{0}+R T \ln c
$$

where $c$ is the solute concentration at steady-state and $\mu_{0}$ the chemical potential of the pure solute. Differentiating with respect to $T$. and substituting $-\bar{S}$ for $\frac{d \mu_{o}}{d T}$ at steady state (equilibrium condition) we obtain 


$$
\frac{d \mu}{d T}=-\bar{S}+R T\left(\frac{d \ln c}{d T}\right)
$$

Again substituting Eq. 39 in 38 and rearranging we obtain

$$
\frac{\mathrm{d} \ln c}{\mathrm{dT}}=\frac{-\mathrm{Q}^{*}}{R \mathrm{~T}^{2}}
$$

Integrating we obtain

$$
\ln c=\frac{Q^{*}}{\mathrm{RT}}+\text { constant }
$$

Equation $40 \mathrm{a}$ is the expression commonly used $(34,35,36)$ to calculate the heat of transport from experimentally determined solute redistribution profiles. The $Q^{*}$ thus calculated is the parameter characterizing the distribution of solute concentration and has generally been taken to be that of a purely irreversible quantity. So, it is considered to be dependent in some way on the details of solute diffusion, but uninterpretable from ordinary thermodynamic considerations. Shewmon (34), however, has suggested from studies of carbon migration in $\alpha$ and $\gamma-\mathrm{Fe}$ that there is a possible equality between $Q^{*}$ and the heat of solution of the solute. Raleigh and Somer (31) beginning with a derivation of the expression for the distribution of solute activity in a real solution showed that $Q^{*}$ may be separated into two parts; the one identifiable with the ordinary heat of solution and the other related to the irreversible effect; thus characterizing the distribution of solute activity rather than the concentration, where the activity is referred to pure solute as the standard state. Hence by reconsidering Eq. 38, we have

$$
\mu=\mu_{0}+R T \ln a
$$


where $a$ is the activity of the solute referred to pure solute as the standard state. At constant pressure, $\mu$ is only a function of a and $T$,

$$
\mathrm{d} \mu=\left(\frac{\partial \mu}{\partial a_{T}}\right)_{T} d a+\left(\frac{\partial \mu}{\partial T}\right)_{a} d T
$$

where $\left(\frac{\partial \mu}{\partial T}\right)_{a}=-S_{0}+R$ ln a and $S_{0}$ is the molar entropy of the pure solute. If $\Delta \overline{\mathrm{H}}$ and $\Delta \overline{\mathrm{S}}$ are the partial molar enthalpy and entropy of solution for the solute, then

$$
R \ln a=\left(\frac{\mu-\mu_{0}}{T}\right)=\left(\frac{\Delta \bar{H}-T \Delta \bar{S}}{T}\right)
$$

and

$$
\left(\frac{\partial \mu}{\partial T}\right)_{a}=\left(\frac{\Delta \bar{H}}{T}\right)-\bar{S}
$$

From Eq. 41

$$
\left(\frac{\partial \mu}{\partial a}\right)_{T}=\frac{R T}{a}
$$

Substituting Eqs. 44 and 45 into 42 and taking $d \mu$. with respect to temperature we have

$$
\frac{d \mu}{d T}=\left(\frac{\Delta \bar{H}}{T}\right)-\bar{S}+R T \frac{\left(d l_{\grave{n}} a\right)}{d T}
$$

Put Eq. $4 \dot{6}$ into 38 and obtain,

$$
\frac{(\mathrm{d} \ln \mathrm{a})}{\mathrm{dT}}=\frac{-\left(\mathrm{Q}_{\mathrm{H} 1}^{*}+\Delta \overline{\mathrm{H}}\right)}{R T^{2}} .
$$


This equation is restricted to neither an ideal nor regular solution. It is therefore a more exact form of Eq. 40a. $\Delta \overline{\mathrm{H}}$ is the partial molar heat of solution and $Q_{H 1}^{*}$ is the thermal gradient effect. 
EXPERDMENTAL PROCEDURE

Equipment

Al1 transport studies were performed in a $304 \mathrm{~L}$ stainless stee 1 chamber with an inside diameter of $9.53 \mathrm{~cm}$ and length of $20.32 \mathrm{~cm}$ (Fig. 2). A viewing port was fitted at the end of the chamber with a quartz sight glass sealed by a neoprene "0-ring". The chamber was held in place by flanges utilizing a neoprene "0-ring" seal. To minimize contamination of the sample, the chamber was evacuated with an oil diffusion pump backed by a mechanical pump (Welch Duo-Seal Model 1397B) for 48 hours, then isolated by a valve and was backfilled with $13 \mathrm{~cm}$ argon pressure. The gas was needed to suppress vaporization of the iron and nickel. The gas was purified by gettering with a zirconium strip measuring $1.27 \times 0.25 \times 5.72 \mathrm{~cm}$ heated to $1350^{\circ} \mathrm{C}$ for 50 hours. "L-shaped" copper adapters supporting the electrotransport couple were fitted into the ends of two copper electrodes and. held in place by set-screws. The electrodes were sealed by a series of neoprene "O-rings" and compressors. Both the chamber and electrodes were cooled by passing water through copper coils which were wound around them. Power was supplied to the sample through the electrodes. A complete schematic of the equipment setup is shown in Fig. 3.

\section{Alloy Preparation}

The iron used in this study was obtained from Glidden Corporation in the form of electrolytic platelets with a reported purity of $99.94 \%$. 


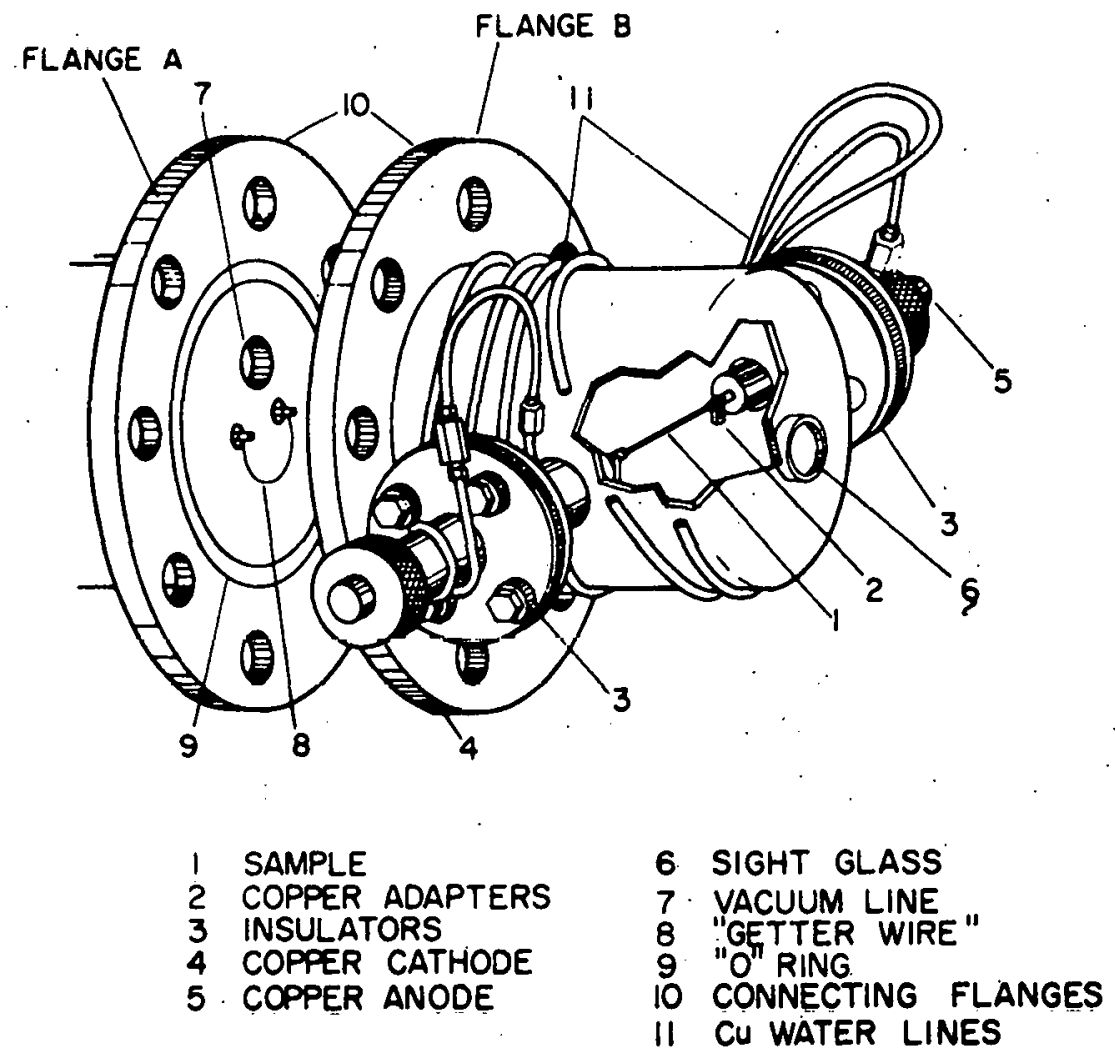

Figure 2. Schematic of the vacuum chamber used in the study. 


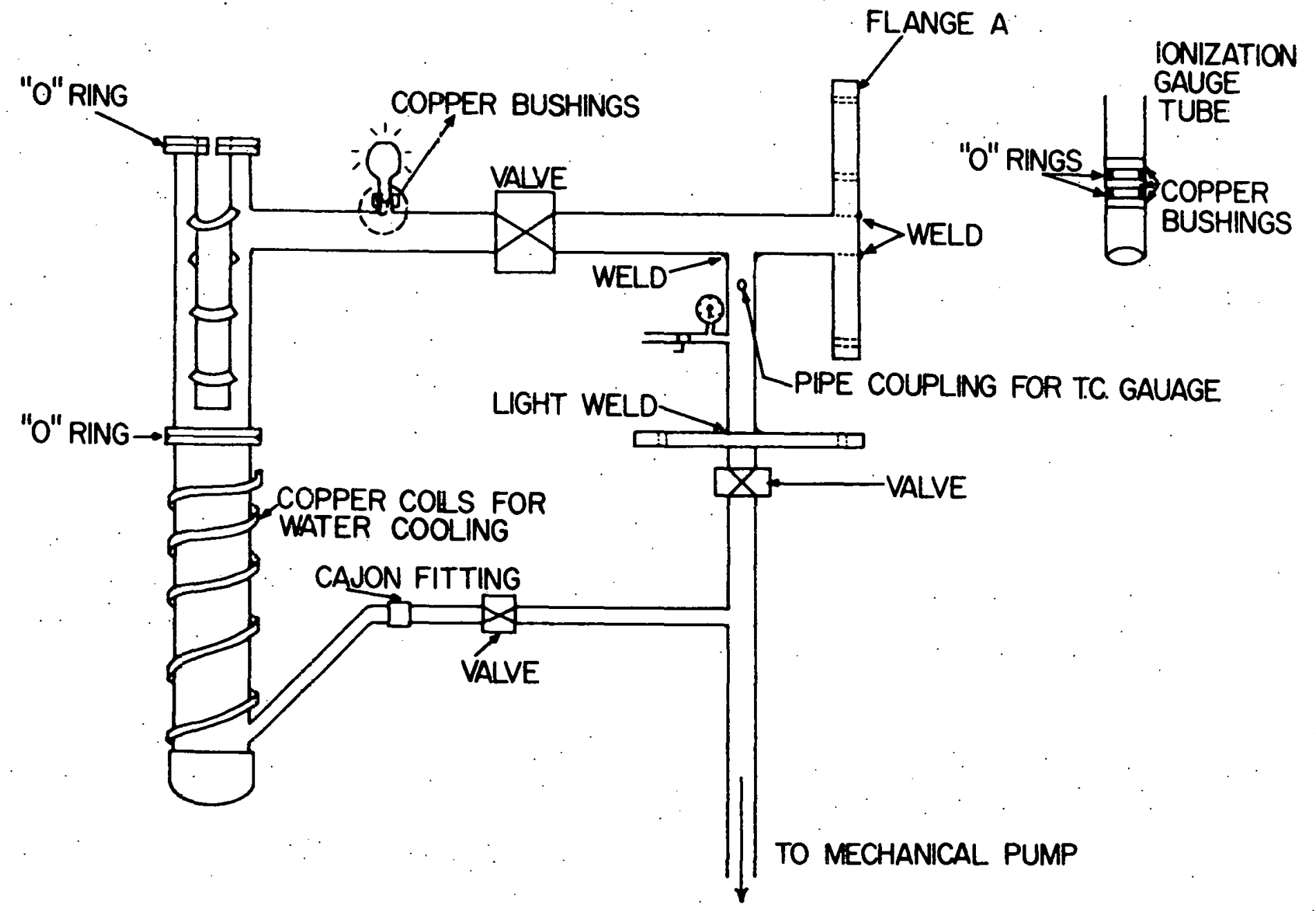

Figure 3. Schematic of the vacuum line. 
Nicke1 rods with a reported purity of $99.98 \%$ obtained from the International Nickel Company were used in this study. Analytical results of the nickel as performed at the Ames Laboratory and the manufacturer's analysis of the Glidden iron are tabulated in Table 2 .

Each of the materials was first melted into a button on a watercooled copper mold under a purified argon atmosphere. The button was then electron beam melted to remove all volatilizable impurities. Experimental alloys containing 32.5 wt \% nickel but different carbon concentrations were then made by arc-melting under purified argon atmosphere. Alloys designated 2 through 9 (Table 3) were submitted for carbon analysis to compare with the nominal composition. From Table 3 it is seen that the results are in good agreement with the nominal composition.

Each button weighed 20-35 gms. Radioactive carbon-14 was added to alloys 2 through 7, while 8 through 11 and duplicate alloys 2 through 9 had no carbon-14 addition. All the specimens were made into "fingers" and homogenized at $1000^{\circ} \mathrm{C}$ for 72 hours in $210^{-6}$ torr vacuum.

Alloys 2 through 7 containing radioactive carbon were swaged to $0.25 \mathrm{~cm}$ diameter wires while the other alloys were swaged to $0.64 \mathrm{~cm}$ diameter rods. The swaging worked the high carbon alloys extensively so that several intermediate anneals were required to prevent cracking of the rods.

A11 swaged rods and wires were degreased in trichloroethane, washed in acetone and rinsed in methanol using an ultrasonic vibrator. 
Table 2. Analysis of materials used in the study

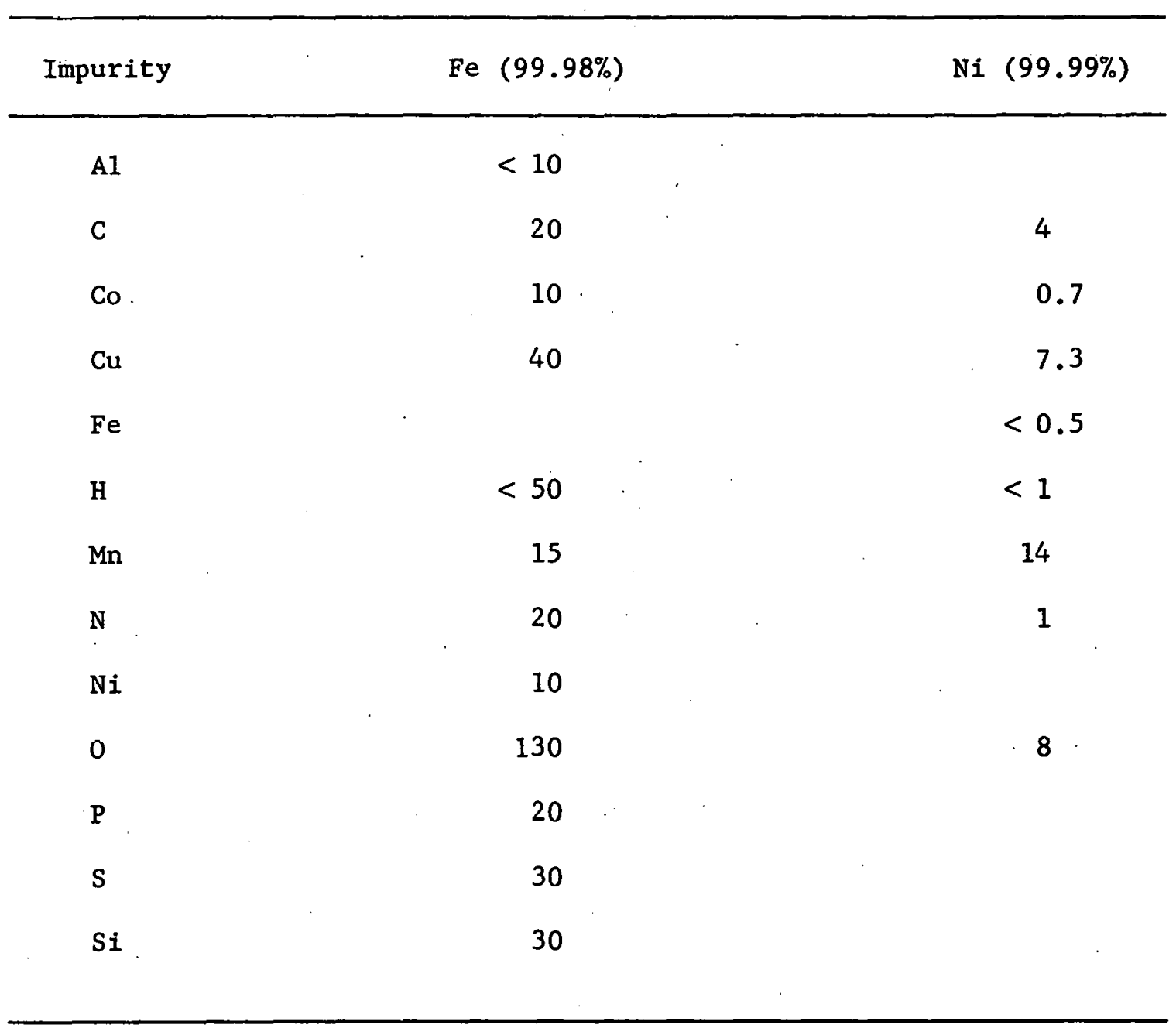


Table 3. Composition of alloys used in investigation

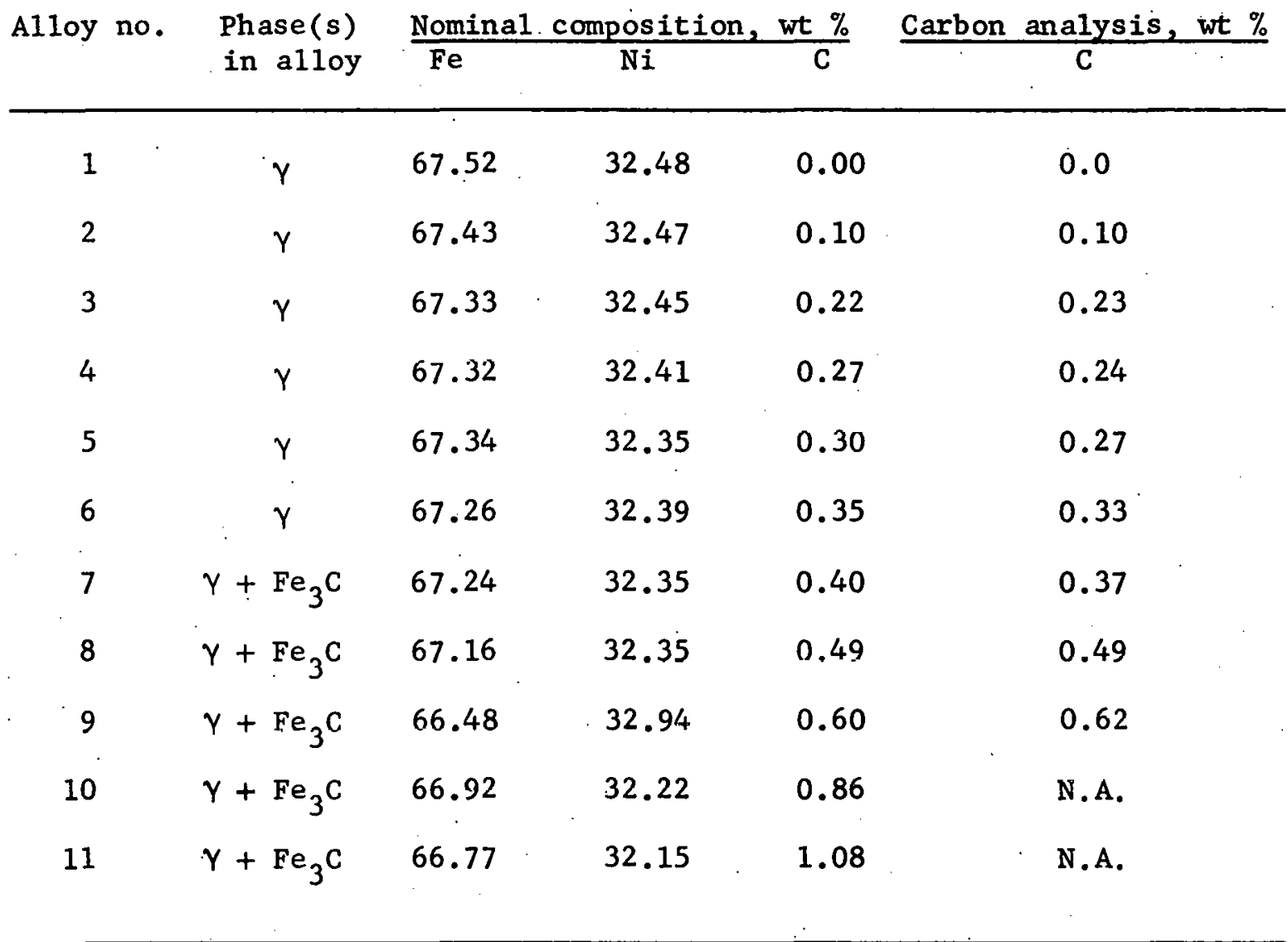


The cleaned specimens were then annealed at $1000^{\circ} \mathrm{C}$ for 24 hours in a vacuum of $10^{-6}$ torr. The annealing was necessary to remove cold work due to swaging and to coarsen the grain structure. Coarse grains were needed to enhance bulk interstitial migration instead of grain boundary migration known to be favored by fine grains.

The $0.25 \mathrm{~cm}$ diameter wires containing radioactive carbon were sectioned into two lengths; namely, $1.0 \mathrm{~cm}$ and $4.4 \mathrm{~cm}$. Alloy no. 1 which had no intentionally added carbon was cut into pieces $2.2 \mathrm{~cm}$ long. The 0.64 diameter rods were cut into small segments about $0.76 \mathrm{~cm}$ long. The $1.0 \mathrm{~cm}$ long pieces were used for thermotransport experiments. The $2.2 \mathrm{~cm}$ and $4.4 \mathrm{~cm}$ long pieces were butt-welded together using a Hughes Aircraft Company discharge welder (Mode1 VTW 30C) at 100 watt -S. The welded samples were used for electrotransport experiments.

The larger diameter pieces were used for carbon solubility studies. Rod forms of alloys $1,3,8$ and 10 swaged to $0.64 \mathrm{~cm}$ diameter were used for resistivity studies. All experimental pieces were electropolished in $6 \%$ perchloric acid in methanol chilled with 1iquid nitrogen to $-70^{\circ} \mathrm{C}$. All butt-welded samples were electropolished prior to welding.

\section{Solubility Studies}

The amount of carbon solubility in the Fe-Ni-C ternary system has not been completely determined. Data of Soehnchen and Piwowarsky (37) indicate that for the temperature range of 800 to $1200^{\circ} \mathrm{C}$ the solubility of carbon in a $Y$-phase Fe-32.5 wt \& $\mathrm{Ni}$ alloy varies from 
approximately 0.3 to $1.0 \mathrm{wt} \%$. The need for more accurate solvus data in this work required that solubility studies be done. An alloy of 0.5 wt \% carbon composition reported to be a single phase at $1000^{\circ} \mathrm{C}$ was . prepared and equilibrated at that temperature for 24 hours and quenched in oil. It was found to contain some carbide. Therefore alloys 2 through 10 (Tab1e 3) with no radioactive carbon were prepared in an attempt to determine the solubility limit more positively. A11 specimens were homogenized in a tantalum resistance furnace at $1100^{\circ} \mathrm{C}$ for 24 hours in a vacuum of $10^{-6}$ torr. Each specimen was then equilibrated at 850 , 950,1050 and $1100^{\circ} \mathrm{C}$ for $120,72,30$ and 96 hours respectively and quenched in oil at room temperature. Quenched specimens were electropolished in a bath of $6 \%$ perchloric acid in methanol chilled with liquid nitrogen to $-70^{\circ} \mathrm{C}$ at current density of $0.2 \mathrm{amp} / \mathrm{cm}^{2}$. These were then electroetched in a $10 \%$ oxalic acid solution. Photomicrographs of the quenched samples were used in quantitative metailography studies to determine the solvus boundary. A nickel line scan (using the SEM) was run on two of the two-phase alloys to determine the major metallic constituent of the carbide precipitates.

\section{Resistivity Studies}

To accurately determine the electric mobility. $U$ and hence the effective valence $z^{*}$, the electric field $E$ must be known. Ohms law states that $E=I R$, where $I$ is current in amperes, $R$ the resistance in ohms and $\mathrm{E}$ is in volts. The resistance of any conductor is known to vary with temperature. Electrical resistance $R$ is related to resistivity 
$\rho$ by the relation $R=\rho \frac{l}{A}$ where $l$ is the length of the conductor and A the cross-sectional area. Any change in $\mathrm{R}$ with temperature is therefore a corresponding change in $\rho$ since $l$ and $A$ are assumed to be constant. For this reason resistivity variation as a function of temperature was investigated for alloys no. $1,2,3,8$ and 10 . The method used in this study was developed by Chiotti (38). A detailed account of the experimental procedure and the relevant schematics can be found in the reference quoted.

In summary, a bar 10 to $12 \mathrm{~cm}$ long swaged to $0.64 \mathrm{~cm}$ diameter had a small black-body hole drilled into it. The bar was mounted between two water-cooled copper electrodes in a vacuum chamber. The potential developed between two probes $1 \mathrm{~cm}$ apart, which made contact with the bar near its midpoint, was balanced against a potential developed across a known resistance in the secondary of a current transformer whose primary was in series with the bar. The transformer maintained a constant ratio between the primary and secondary currents and consequently the resistance of the $1 \mathrm{~cm}$ portion of the bar between the probes is directiy proportional to the known resistance in the secondary. The temperature of the heated bar was measured with a disappearing filament optical pyrometer.

\section{Sources of errors}

The distance $\mathrm{L}$, between the potential probes that make contact with the specimen needs to be known very accurately because it contributes to the value of the resistivity from the relation $\rho=\left(\frac{A}{L}\right) R$. 
Chiotti (38) estimates this error to be between 1 and $2 \%$. The recorder sensitivity and readings could be affected by induced ac components in the measuring circuit particularly in the potential leads from the specimen. This was minimized by keeping the leads as short as possible.

\section{Electrotransport Studies}

Electromigration measurements were made on two alloys, nos. 2 and 3 (Table 3). Experiments were performed in a vacuum chamber evacuated to a pressure of about $10^{-6}$ torr. The chamber was then backfilled with argon and purified of oxygen and other reactive gases by gettering with a zirconium strip heated to $1350^{\circ} \mathrm{C}$ for 50 hours.

A dc electric field was applied to heat the butt-welded samples to temperatures of $850,950,1050$ and $1200^{\circ} \mathrm{C}$ for $96,72,24$ and 1 hour(s) respectively. The temperature measurements were made with a Leeds and Northrup optical pyrometer of the disappearing filament type. Temperatures were corrected for both emissivity of the alloy and the sight glass absorption of the quartz viewing port. To determine the emissivity correction factor, $0.03 \mathrm{~cm}$ diameter holes $0.18 \mathrm{~cm}$ deep and approximately $2 \mathrm{~mm}$ apart were drilled into alloys nos. 2 and 3 . The rods were heated between two water-cooled copper electrodes using an ac power supply. The imposed temperature gradient gave a distribution of temperatures. The holes served as black-body temperature sources which were compared with the temperature of the adjacent metal surface. 
The two sets of temperatures were fitted on a straight line using a least squares fit to the form $T=m T_{a}+K$ where $T$ is the true temperature, $T_{a}$ the observed temperature of the metal surface, $K a$ constant and $\mathrm{m}$ is the slope of the straight line. Following an electrotransport run samples were sectioned into about thirty pieces each about $1.5 \mathrm{~mm}$ long. Activity counts of the tracer carbon-14 were taken for each side of the thirty sample pieces. Counting was done with the aid of a Baird Atomic Abacus GM Scale (Mode1 123A) radioactive counter.

\section{Determination of Electrotransport Parameters}

The differential equation describing unidirectional electrotransport of solute atoms in a solvent can be written as

$$
\frac{\partial c}{\partial t}=\partial\left(D \frac{\partial c}{\partial x} \pm U E c\right) \partial x
$$

where $c$ is the solute concentration, $D$ the diffusivity, $x$ the migration distance; $U$ its electric mobility in the solvent and $E$ the electric. field. For a case where $E$ is along the axis of the rod and is constant throughout the length of the rod, then for a dilute solution where U and $\mathrm{D}$ can be assumed to be constant, the differential equation becomes

$$
\frac{\partial e}{\partial t}=D \frac{\partial^{2} c}{\partial x^{2}} \pm \text { UE } \frac{\partial c}{\partial x}
$$


The initial conditions for this equation are

$$
\begin{aligned}
& C_{i}=C_{L} \text { for } x<0 \text { and } t=0 \\
& C_{i}=C_{H} \text { for } x>0 \text { and } t=0
\end{aligned}
$$

where $C_{L}$ is the low solute concentration, $C_{H}$ is the high solute concentration and $C_{i}$ is some solute concentration along the diffusion couple. Since each couple is long relative to its diameter $(66 \times 0.25 \mathrm{~mm})$ each segment can be considered to be semi-infinite in length. The boundary conditions then become

$$
\begin{aligned}
& C_{i}=C_{L} \text { for } x=-\infty \text { and } t>0 \\
& C_{i}=C_{H} \text { for } x=\infty \text { and } t>0 .
\end{aligned}
$$

Mason and Weaver (39) using these initial and boundary conditions obtained the solution to the differential equation as

$$
C_{i}=C_{L}+\frac{C_{H}-C_{L}}{Z}\left(1+\operatorname{erf}\left(\frac{x \pm U E t}{2 \sqrt{D t}}\right)\right)
$$

wherc crf is the error funetion defined by

$$
\operatorname{erf}(\mu)=\frac{1}{\sqrt{\pi}} \int_{-\mu}^{\mu} e^{-x^{2}} d x .
$$

Recalling that UE is the velocity of transport due to electrotransport, then it can be seen that

$$
x \pm U E t=x \pm V^{T} t=x \pm \Delta x^{T}
$$


where $\Delta \mathrm{x}^{\mathrm{T}}$ is the total distance the atoms have moved as a result of electrotransport.

In the absence of a dc electric field, only chemical diffusion would occur. For this condition, the solute concentration at the weld interface between the high and low solute segments would be their mean, $\frac{\left(\mathrm{C}_{\mathrm{H}}+\mathrm{C}_{\mathrm{L}}\right)}{2}$. During electrotransport, however, this interface is shifted towards either the anode or the cathode by an amount $\Delta x^{T}$, thus placing the mean at a new "displaced" interface. Since the velocity of transport of the solvent atoms is approximately zero relative to interstitial electrotransport, the weld interface serves as a convenient reference with respect to the displaced interface in determining the amount of displacement, $\Delta x^{T}$. A solution to Eq. 47 involves finding the values of $\Delta x^{T}$, and $D$, the diffusivity. $D$ and $\Delta x^{T}$ are used to evaluate the other electrotransport parameters $U$ and $z^{*}$. Carlson et al. (40) obtained $\mathrm{D}$ and $\Delta \mathrm{x}^{\mathrm{T}}$ by a graphical technique. This same method was used in this study. A plot of solute concentration against distance gives the interface displacement $\Delta x^{T}$. D is obtained from a plot of the inverse error function of the solute concentration versus distance and the slope, $m$, of the straight line is $\left(\sqrt{4 \mathrm{Dt}}^{-1}\right.$. From a plot of $\ln D$ versus $\frac{1}{T}$, the activation energy $Q$, for diffusion is obtained from the slope of the straight line, and the intercept on the vertical, $\ln D$, axis gives the value of $D_{0}$. 
Thermotransport and Determination of $Q^{*}$

A steady state technique was used to determine the heat of transport in a series of alloys of different carbon concentrations. Alloys 3 and 7 containing nominal 0.2 and 0.4 wt $\%$ carbon, respectively were investigated primarily to determine the concentration dependence of $Q^{*}$. Alloy 9 containing 0.6 wt $\%$ carbon was selected primarily to study the combined effects of "true" thermotransport and chemical diffusion of this solute due to a variation of solute solubility over the temperature range in question. For the temperature range of interest, 850 to $1100^{\circ}$, the results of previous investigators $(37,41)$ indicate that an 0.6 wt \% C alloy (非) crosses the solvus boundary. The samples used were $1.0 \mathrm{~cm}$ long and $0.25 \mathrm{~cm}$ diameter. Experiments were carried out in the same vacuum chamber as was used for the electrotransport studies, except that the electrodes were water cooled in the latter case. Each sample was run in a purified argon atmosphere using an alternating current. The argon was needed to suppress metal ( $\mathrm{Fe}, \mathrm{Ni}$ ) vaporization. Each experimental piece was subjected to a temperature gradient for a long enough time to achieve steady state.

The time to establish steady state is proportional to exp $\left(\frac{-t}{\theta}\right)$, where " $t$ " is the time of the run and $\theta=\frac{a^{2}}{\pi^{2} D}$ (34) is called the relaxation time. "a" is the specimen dimension in the direction of the temperature gradient and $D$, the solute diffusivity at the mean run temperature. Theoretically, infinite time is required to attain ultimate steady state conditions. But for this work each experiment 
was run for $t=5 \theta$. Thus, the final state is approached to within $\exp (-5)$ which is 0.0063 or $99.3 \%$, which is quite satisfactory for determining $Q^{*}$. From Eq. 36 a plot of $\ln$ c versus $\frac{1}{T}$ yields a straight line whose slope is $\frac{Q^{*}}{R}$.

The run specimens were, sectioned into a number of pleces about $0.05 \mathrm{~cm}$ thick. Carbon concentrations in the sectioned pieces were determined by making an activity count using the Baird Counter described earlier or by chemical analysis. 
EXPERIMENTAL RESULTS

The results are presented in four sections: Solubility, Resistivity and Electrotransport studies and Thermotransport and Determination of $Q^{*}$. In order to study the effect of carbon concentration and thermotransport over a wide concentration range it was necessary to know the solubility boundary for carbon in Fe-32.5 wt $\% \mathrm{Ni}$ quite accurately. The resistivity change with temperature is needed to determine the electrotransport parameters $U$ and $z^{*}$ at various temperatures. A poorly-understood anomaly in the behavior of $Q^{*}$ in an alloy that crosses the solvus boundary is discussed in the thermotransport section.

\section{Solubility Studies}

A plot of the solubility limit for carbon in $\mathrm{Fe}-32.5 \mathrm{wt} \% \mathrm{Ni}$ as a function of temperature as determined in this investigation is shown in Fig. 4. A second curve based on data from the literature is also shown in the figure. The curve obtained from this study shows a somewhat lower, solubility limit fur the cemperature range 850 to $1100^{\circ} \mathrm{C}$. We find a one-phase microstructure for an 0.25 wt $\% \mathrm{C}$ alloy as furnace cooled after annealing at $1000^{\circ} \mathrm{C}$ (Fig. 5a) and an almost one-phase structure for an 0.33 wt $\% \mathrm{C}$ sample quenched from $850^{\circ} \mathrm{C}$ (Fig, 5b). Fig. 6 a shows a two-phase microstructure of the 0.86 wt \% C alloy as equilibrated at and quenched from $950^{\circ} \mathrm{C}$, whereas Fig. $6 \mathrm{~b}$ shows the same alloy as equilibrated and quenched from $1100^{\circ} \mathrm{C}$. It will be noted 


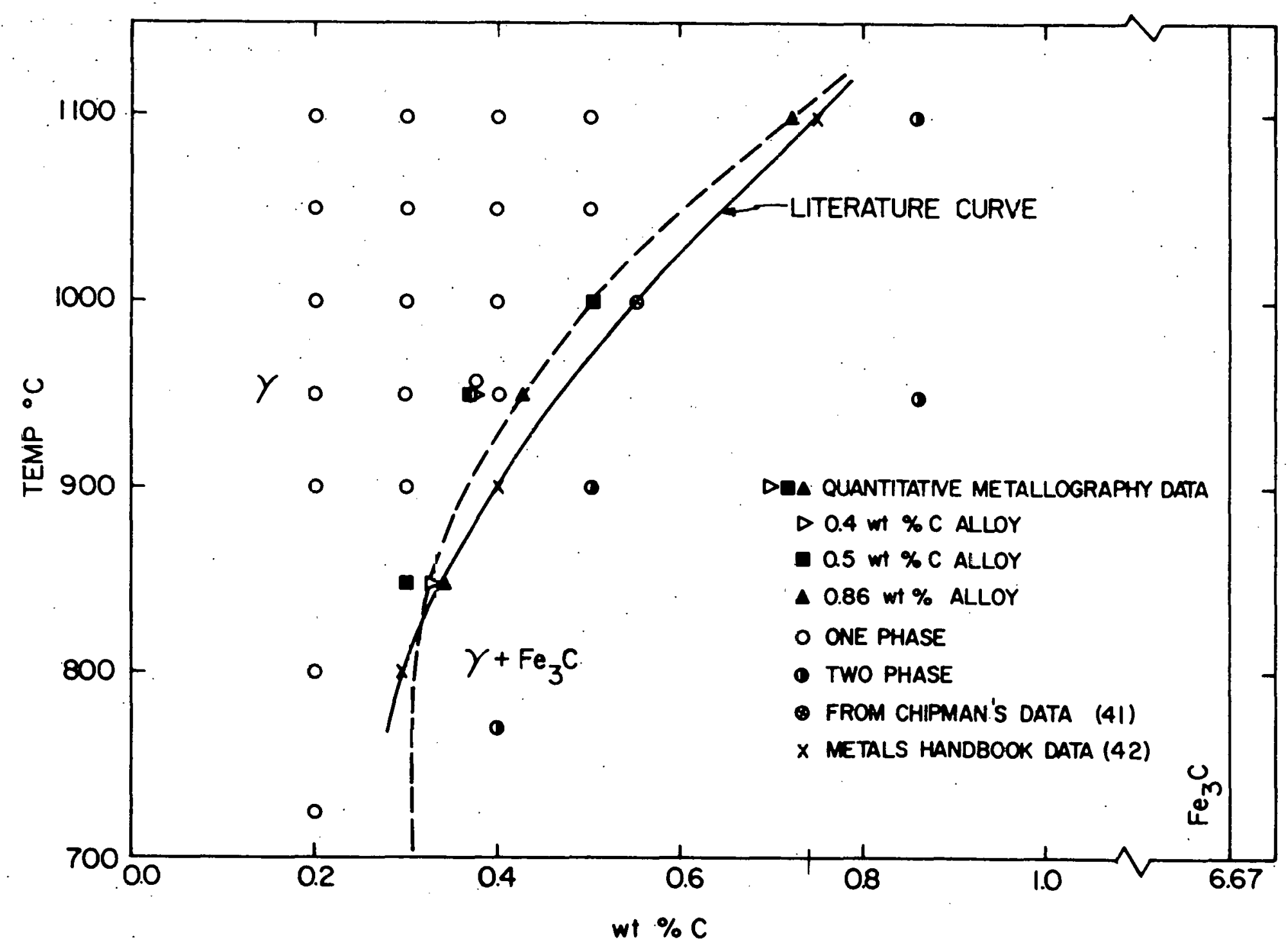

Figure 4. Carbon solubility limit in Fe-32.5 wt \% $\mathrm{Ni}$. 


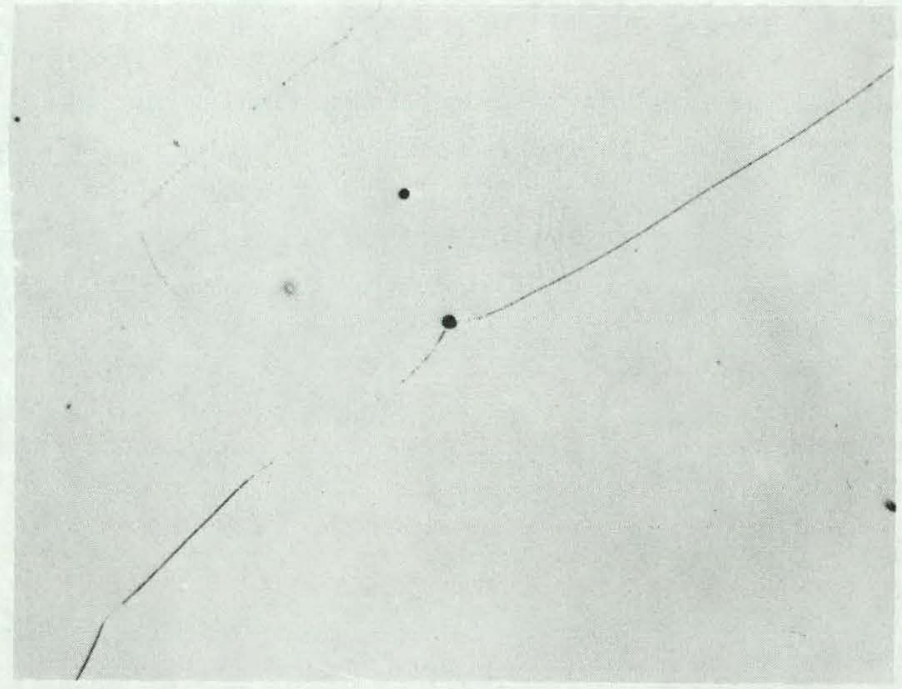

Figure 5a. Single-phase microstructure of Fe-32.5 wt \% Ni 0.25 wt $\% \mathrm{C}$ at room temperature.

Figure 5b. Alloy 非, Fe-32.5 wt $\% \mathrm{Ni}-0.33$ wt $\% \mathrm{C}$ equilibrated at $850^{\circ} \mathrm{C}$, almost single phase. 


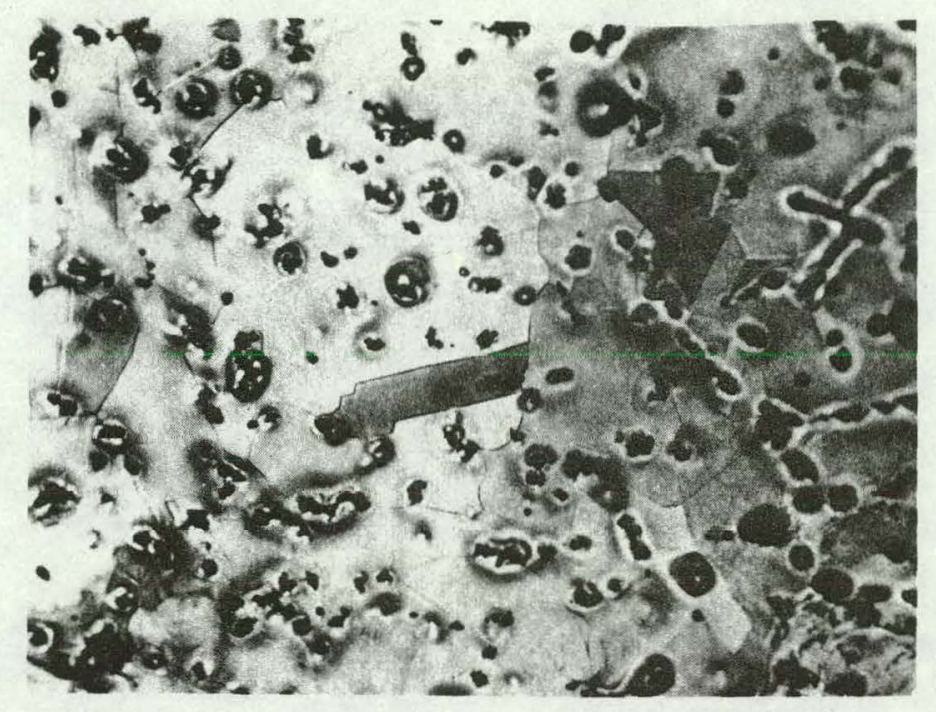

Figure 6a. Microstructure of $\mathrm{Fe}-32.5$ wt $\% \mathrm{Ni}-0.86$ wt \% C a11oy quenched from $950^{\circ} \mathrm{C}$. $7 \% \mathrm{Fe}_{3} \mathrm{C}$ in $\mathrm{Y}$-matrix. Mag. $250 \mathrm{X}$.

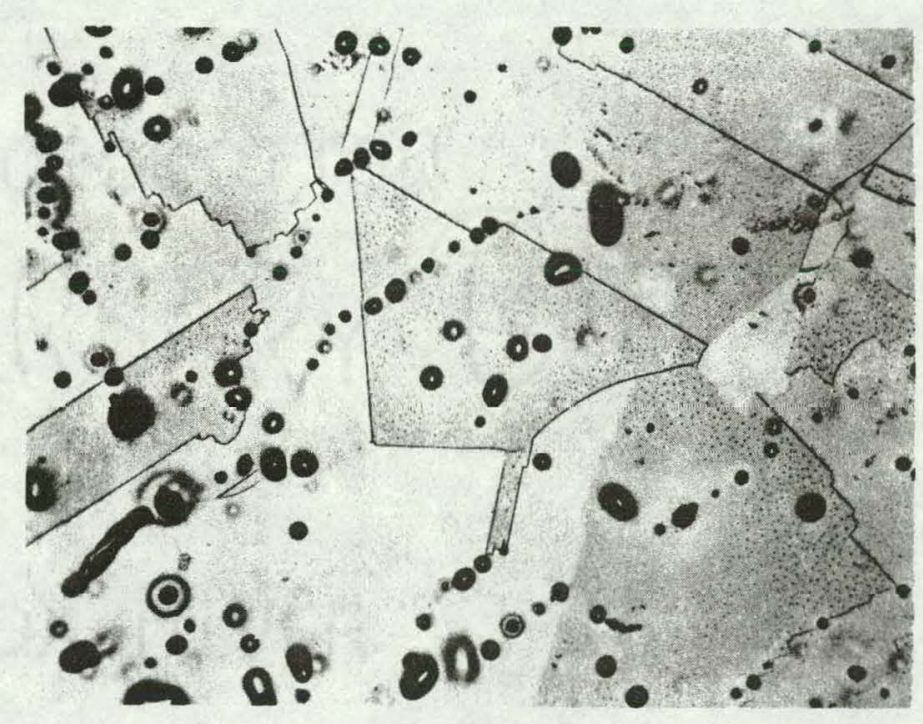

Figure 6b. Two-phase microstructure of Fe-32.5 wt $\% \mathrm{Ni}-0.86$ wt $\%$ $\mathrm{C}$ alloy quenched from $1100^{\circ} \mathrm{C} .2 .2 \% \mathrm{Fe}_{3} \mathrm{C}$ in $\mathrm{\gamma}$-matrix. Mag. 250x. 
that the amount of second phase decreases markedly upon quenching from a high temperature. In our quantitative metallography counts the round, dark region surrounding the precipitate particles were interpreted as the results of over-etching. To identify the precipitate particles a nickel line scan and iron and nickel (SEM) maps were made on the furnace-cooled 0.86 wt $\% \mathrm{C}$ alloy. The line scan indicated a virtual absence of nickel in the precipitate particles (Fig. 7a). The map of nickel distribution (Fig. $7 \mathrm{~b}$ ) of the same sample section likewise showed a low nickel concentration in the same particle. An iron map of the same region showed no iron deficiency. On the basis of this evidence the precipitate particle has been assumed to be $\mathrm{Fe}_{3} \mathrm{C}$. The solvus was therefore determined by application of the lever rule to the various quench samples assuming $\mathrm{Y}-\mathrm{Fe}_{3} \mathrm{C}$ phase equilibria. Considering the uncertainties in the quantitative metallography results, there appears to be fairly good agreement between the solubility results obtained from this study and that of earlier work in the literature.

\section{Resistivity Studies}

The results of the resistivity variation as a function of temperature are shown in the curves in Fig. 8. The room temperature resistivity for the nominal 0.0 wt \% C alloy (非) was determined to be $81.87 \times 10^{-6} \mathrm{ohm} / \mathrm{cm}$ which is in excellent agreement with the value $81.7 \times 10^{-6} \mathrm{ohm} / \mathrm{cm}$ obtained from the resistivity versus wt $\% \mathrm{Ni}$ in $\mathrm{Fe}-\mathrm{Ni}$ alloys, Fig. 9. The room temperature resistivity was measured by passing a known current through a known length of the experimental 


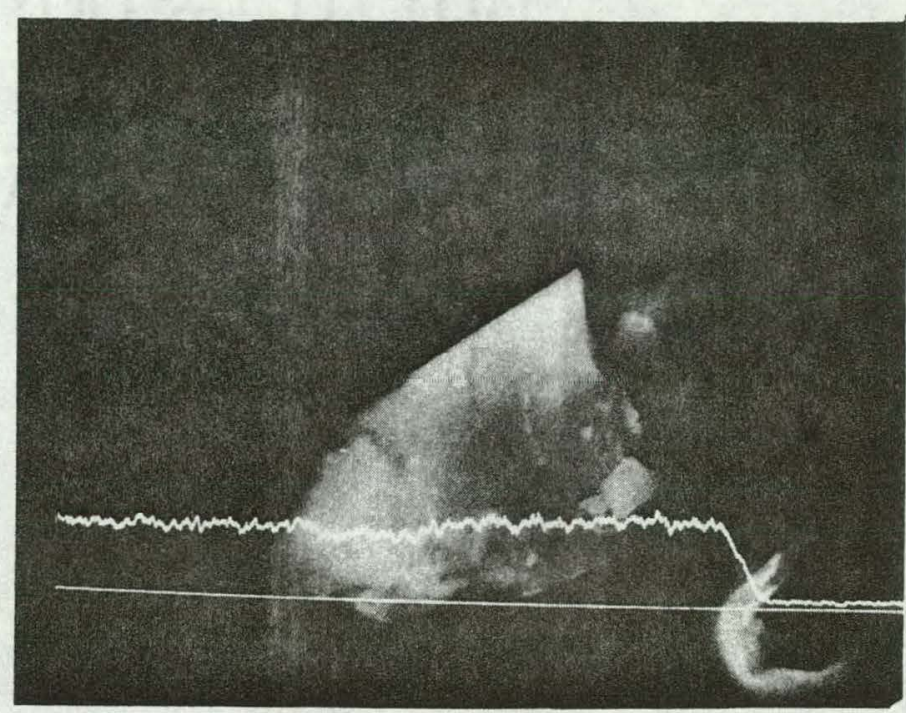

Figure 7a. Nickel line scan (SEM) indicating nickel deficiency in a carbide particle. Mag. 3000X.

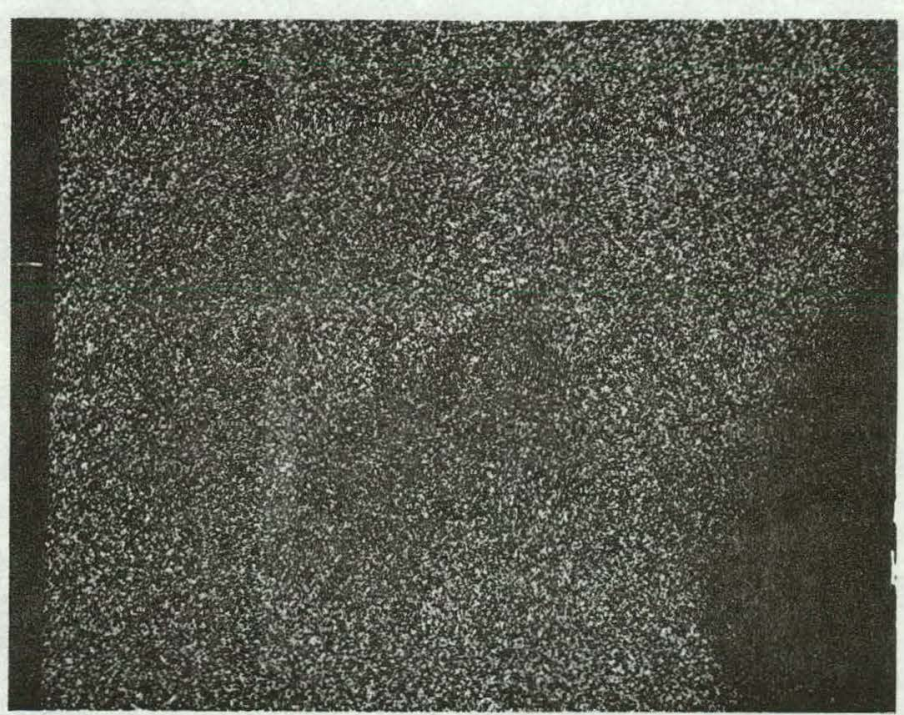

Figure 7b. SEM map of nickel distribution showing extremely low nickel content in the same carbide precipitate as in $7 a$. 


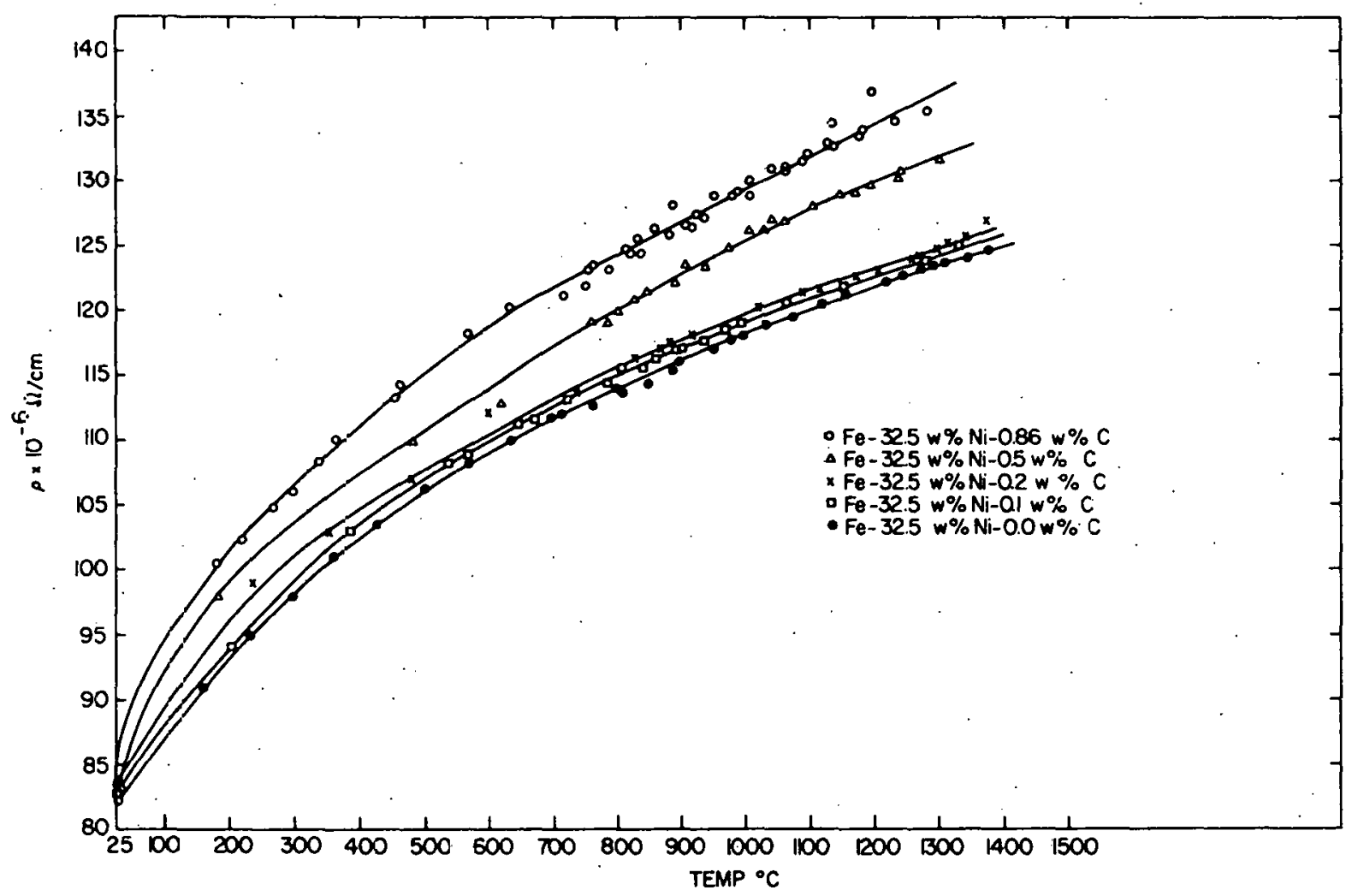

Figure 8. The variation of resistivity as a function of temperature for Fe-Ni alloys: 


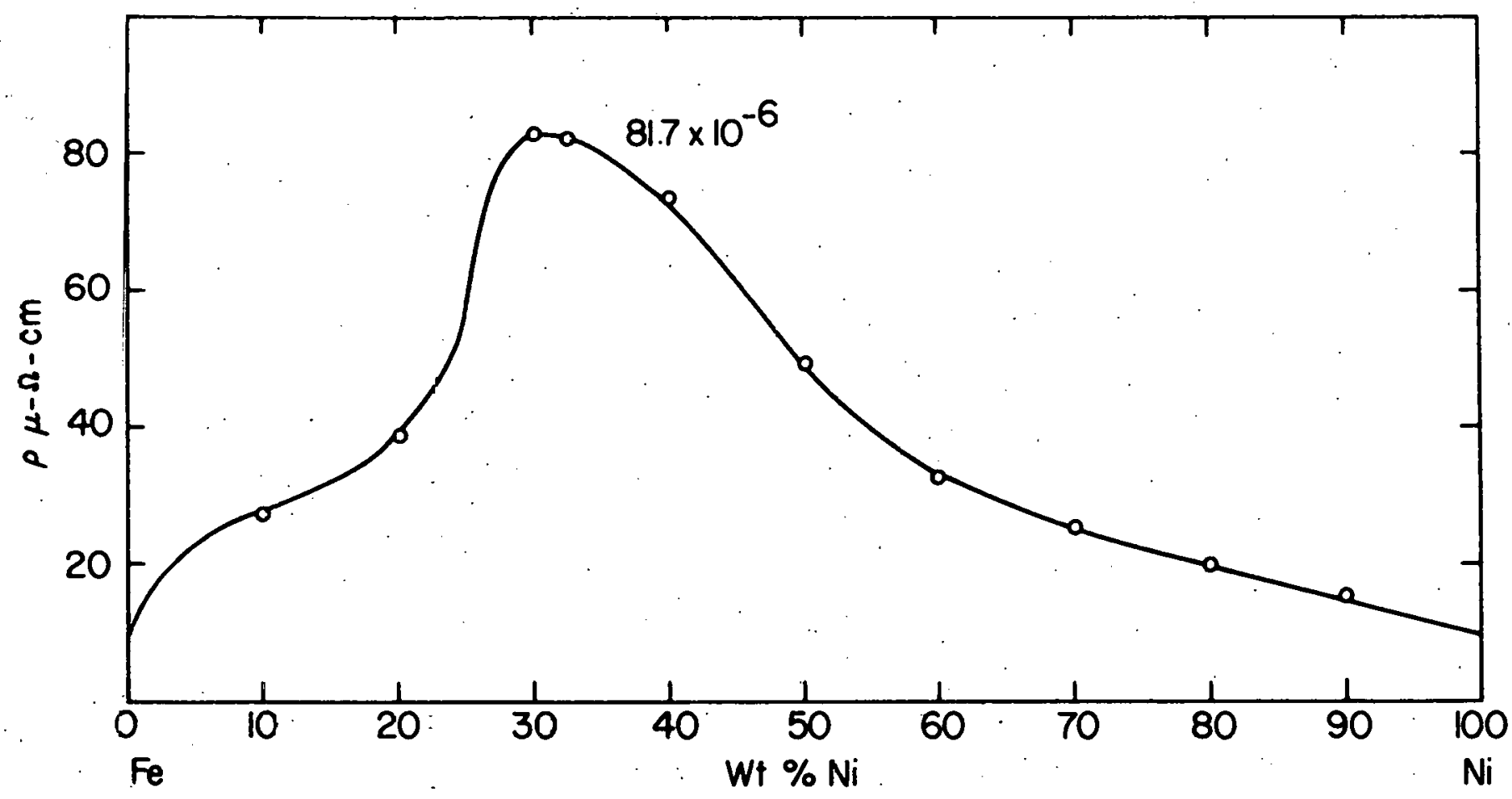

Figure 9. The resistivity variation as a function of nickel content in $\mathrm{Fe}-\mathrm{N} 1$ alloys at room temperature (43a). 
specimen and measuring the resulting voltage drop across this length of specimen. A check was run on the room temperature resistivity results by indirectly extrapolating the high temperature results to room temperature. Two recorders were used in the study. The variation of resistivity as a function of time was plotted on one recorder. The other showed the variation of temperature in millivolts (from a thermocouple placed near the middle of the specimen) as a function of time. The "true" temperature of the specimen measured with a pyrometer was corrected for both sight glass and emissivity and indicated on both recorder charts at specific intervals. A plot of "true" temperatures as a function of millivolt temperatures was made for each specimen studied. A typical temperature versus millivolt plot is shown in Fig. 10. Because lower temperatures than $700^{\circ} \mathrm{C}$ could not be measured directly from the pyrometer, Chiotti (38) suggests extrapolating the linear plot obtained at the high temperature regime to the lower temperature zone. Often this plot is of the form $y=m x+c$. Chiotti (38) further observes that since the millivolt temperature of the thermocouple is obtained from radiant heat from the specimell, this temperature should be zero when no current passes through the experimental specimen. This accounts for the lower part of the plot passing through "zero". From Fig. 10 the "true" temperature for a known millivolt temperature and hence time is obtained. The corresponding time on the "time" versus resistivity chart gives the temperature variation with resistivity for any temperature. The lower 


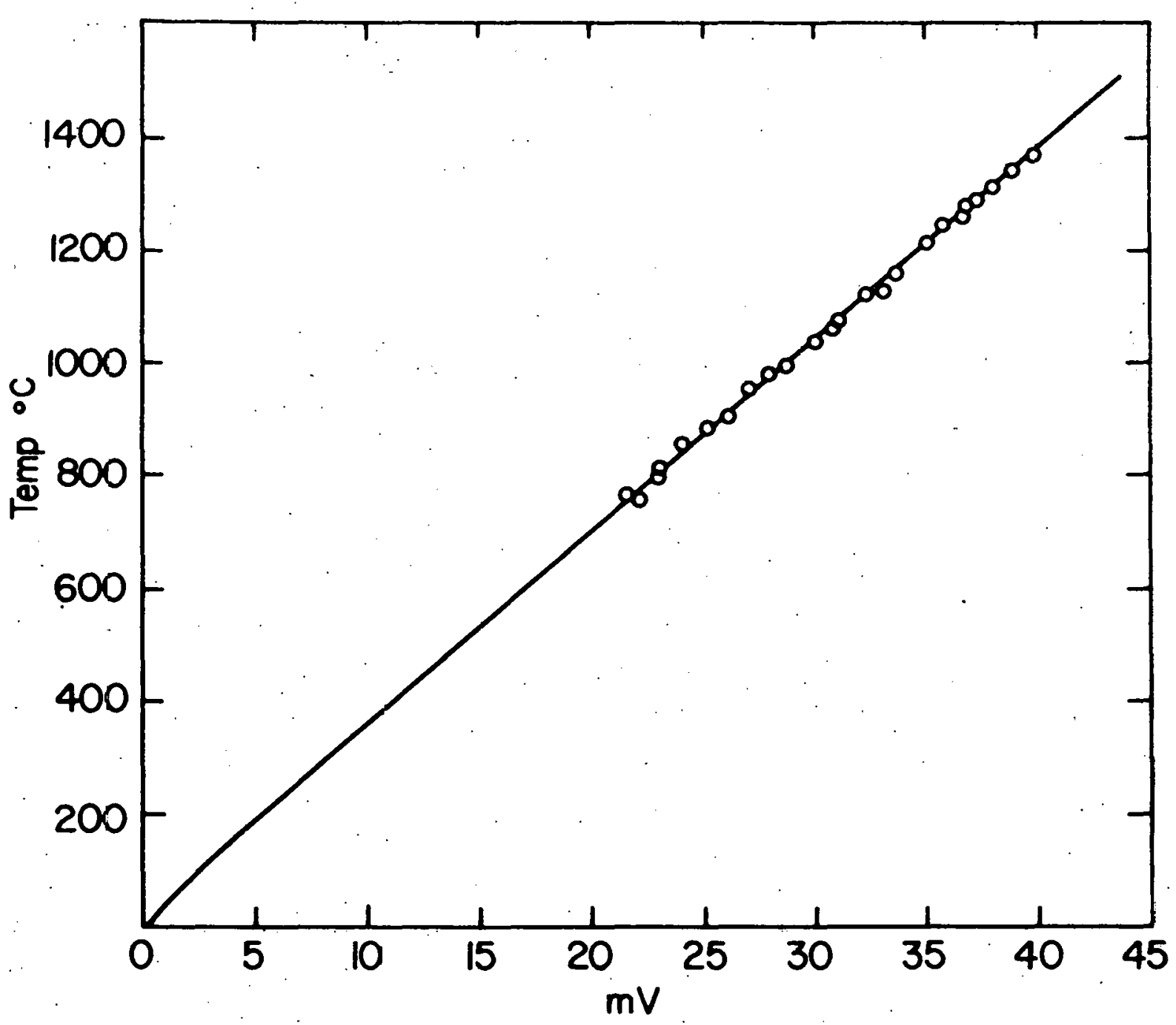

f

Figure 10. The pyrometer temperature versus thermocouple e.m.f. 
temperature data therefore have more inherent errors than their higher temperature counterparts.

The plot of resistivity as a function of temperature as shown in Fig. 8 indicates that at room temperature the resistivity increases with carbon concentration for alloys containing 0.0 to 0.2 wt $\% \mathrm{C}$. In the higher carbon alloys ( 0.5 and 0.86 wt \%), the room temperature resistivities changed very little from the resistivity of the 0.2 wt \% C alloy. This behavior suggests that the solubility limit of carbon in the $\mathrm{Fe}-\mathrm{Ni}-\mathrm{C}$ system at room temperature is exceeded by carbon concentrations of 0.5 wt $\%$ and over. This observation is supported by both reported solubility data and metallographic results in this study. As more solute dissolves into the single phase lattice, the solute atoms act as extra scattering centers as they distort the perfection of the lattice. The resistivity therefore increases. When the solubility limit for any temperature is reached, the maximum resistivity for that temperature is attained and any further solute addition does not change the resistivity by much, since the excess atoms do not go into solution and so do not contribute to the electron scattering.

The curves for the 0.0 to 0.2 wt \% C alloys are approximately parallel from room temperature to $1375^{\circ} \mathrm{C}$. This indicates complete solid solubility over the temperature range. The resistivity curves of the higher carbon alloys also increase monotonically with temperature, but unlike the low carbon alloy curves, these are not paralle1. The curves show a divergence as the temperature increases. 
This suggests that more carbon atoms go into solution as the temperature increases thus increasing the resistivity.

\section{Electrotransport Studies}

Electrotransport experiments were performed on the 0.1 and 0.2 wt $\%$ alloys at approximately $1220,1310,1365$ and $1580^{\circ} \mathrm{K}$. A11 the experiments yielded concentration profiles of an S-shape as shown in Figs. 1la through 18a. From all the S-plots the direction of migration of the carbon solute can be inferred by a "piling up" at one electrode or the other. Plots of the inverse error function versus distance are shown in Figs. 11b through 18b. The linearity of the data is obvious. It must be pointed out that progressing towards the ends of the transport rod the error associated with each datum point increases. This problem arises from making the best linear fit on a non-linear (S-) plot. In effect the reparameterization of the equation of an s-curve to yield an equation for the error function plot overemphasizes the uncertainties at both maximum and minimum portions of the S-plot (43). For this inherent mathematical limitation, most of the end points were neglected in making the least squares fit to the data. The electric mobility was obtained from the relation $U=\Delta x / E t$, Eq. 10, where $\Delta x / t$ is the migration velocity in $\mathrm{cm}^{-1}$ and $E$ the electric field in $\mathrm{V} \mathrm{cm}^{-1}$. The electric field was calculated as the product of the current density and the resistivity of the alloy at the temperature of interest. The values of $U$ obtained for carbon in the two alloys at the four temperatures are given in Table 4 . The table 


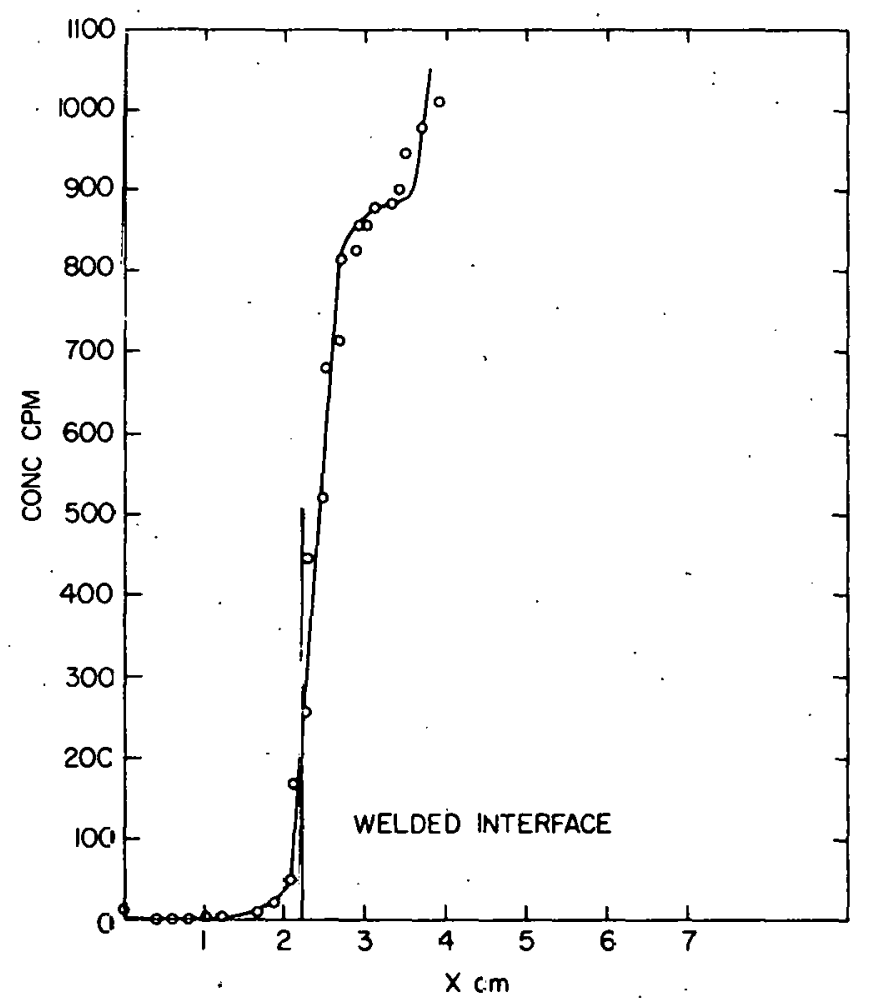

Figure 11a. Concentration versus distance plot for the 0.1 wt \% C alloy after $111.5 \mathrm{hrs}$. at $1220^{\circ} \mathrm{K}$.

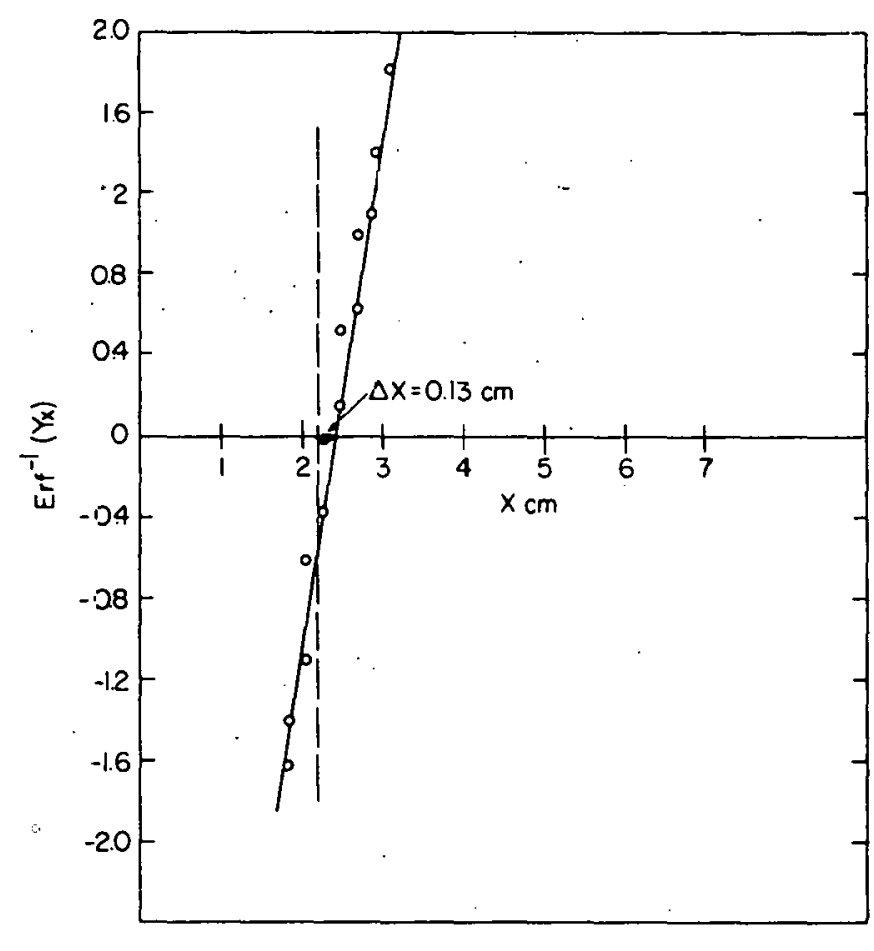

Figure 11b. Inverse error function versus distance $(X \mathrm{~cm})$ plot for the 0.1 wt $\% \mathrm{C}$ alloy run at: $1220^{\circ} \mathrm{K}$ for $111.5 \mathrm{hrs}$. 

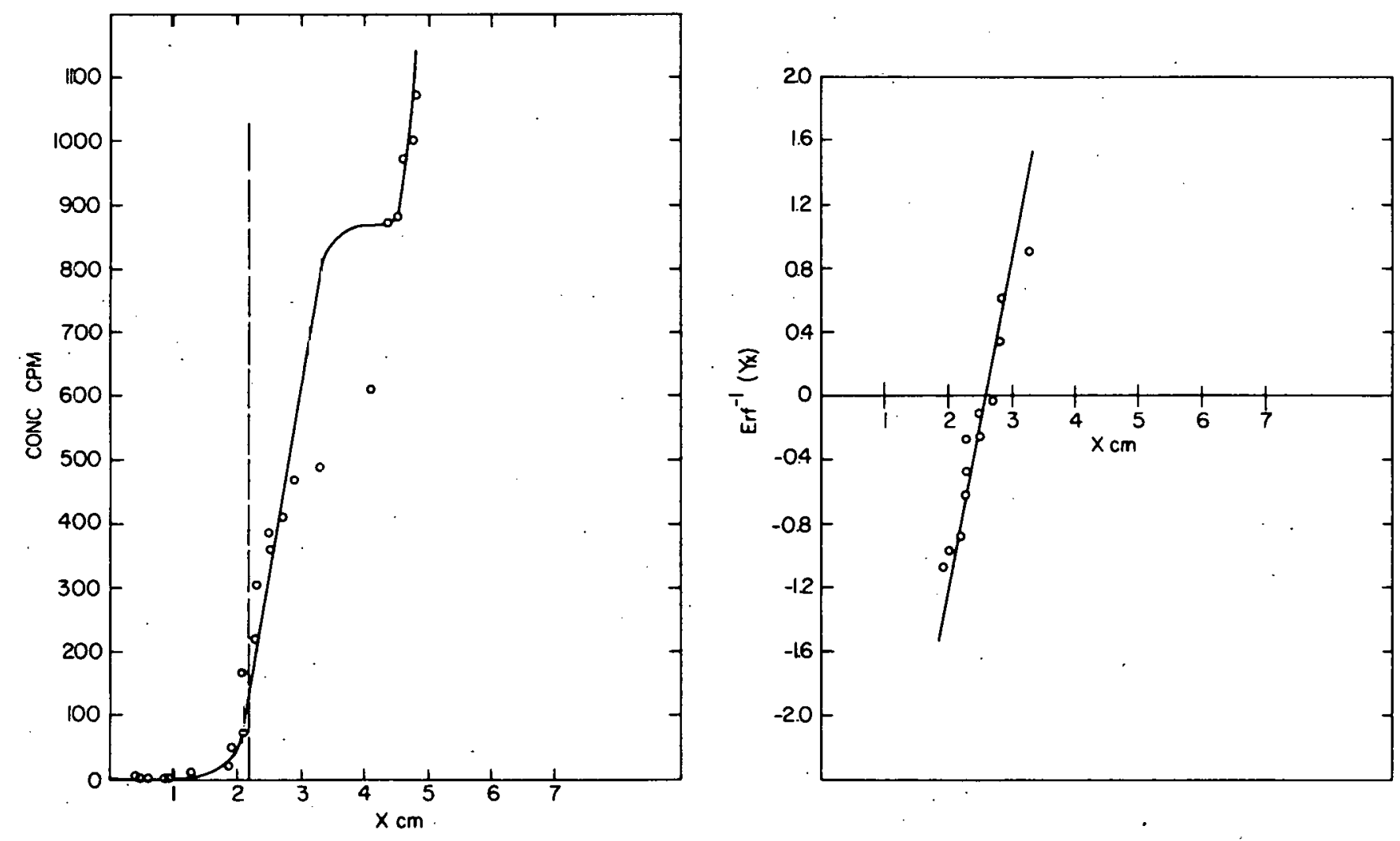

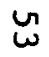

Figure 12a. Concentration versus distance (X cm) plot for the 0.1 wt \% C alloy run at $1313^{\circ} \mathrm{K}$ for $48 \mathrm{hrs}$.
Figure 12b. Inverse error function plot versus distance $(X \mathrm{~cm})$. for the 0.1 wt $\% \mathrm{C}$ alloy run at $1313^{\circ} \mathrm{K}$ for $48 \mathrm{hrs}$. 


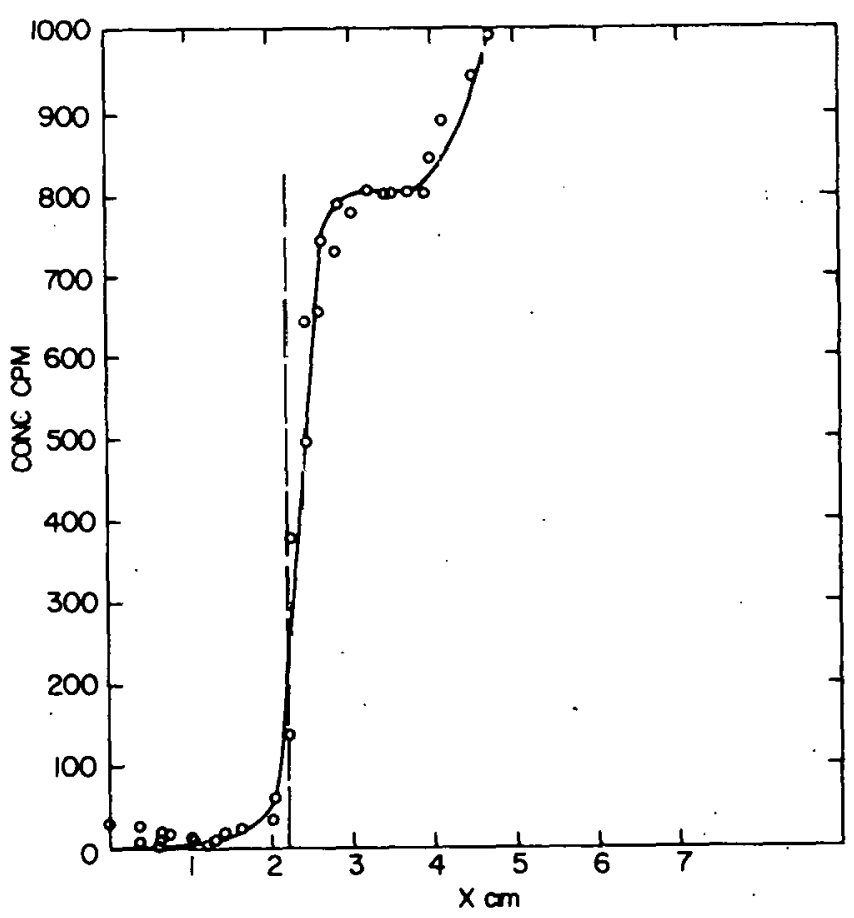

Figure 13a. Concentration versus distance plot for the -0.1 wt \% C alloy run at $1361^{\circ} \mathrm{K}$ for $8 \mathrm{hrs}$.

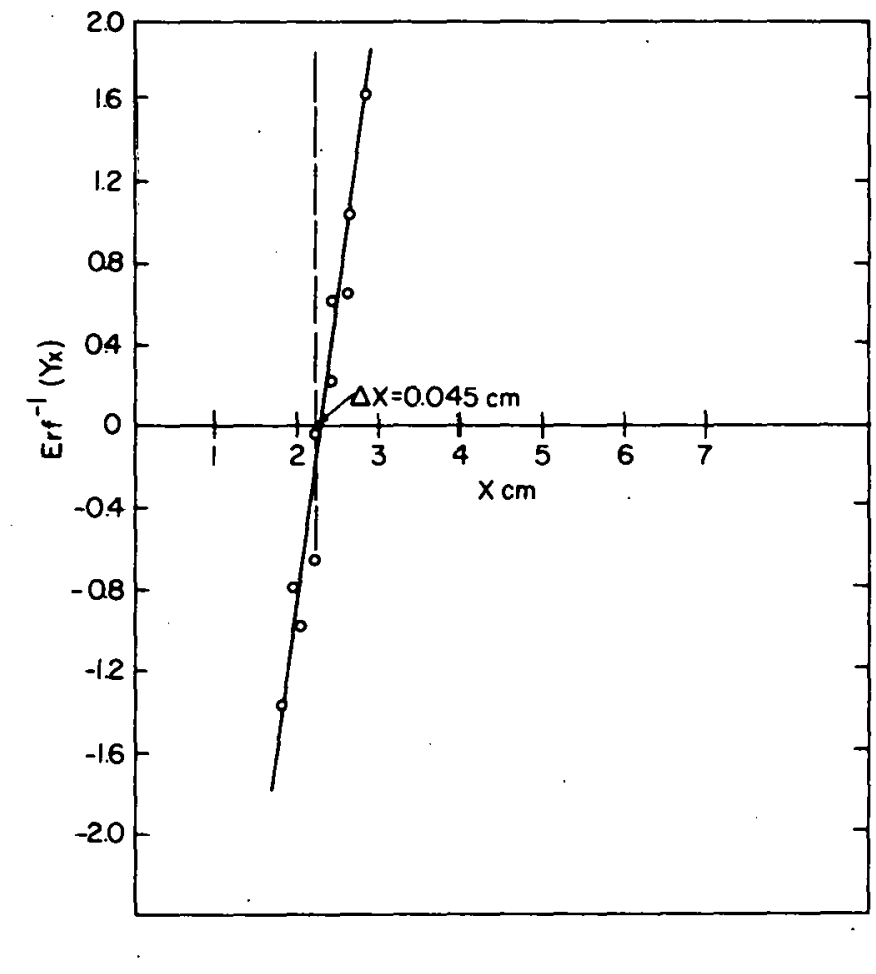

Figure 13b. Inverse error function versus distance plot for -0.1 wt $\% \mathrm{C}$ alloy run at $1361^{\circ} \mathrm{K}$ for $8 \mathrm{hrs}$. 


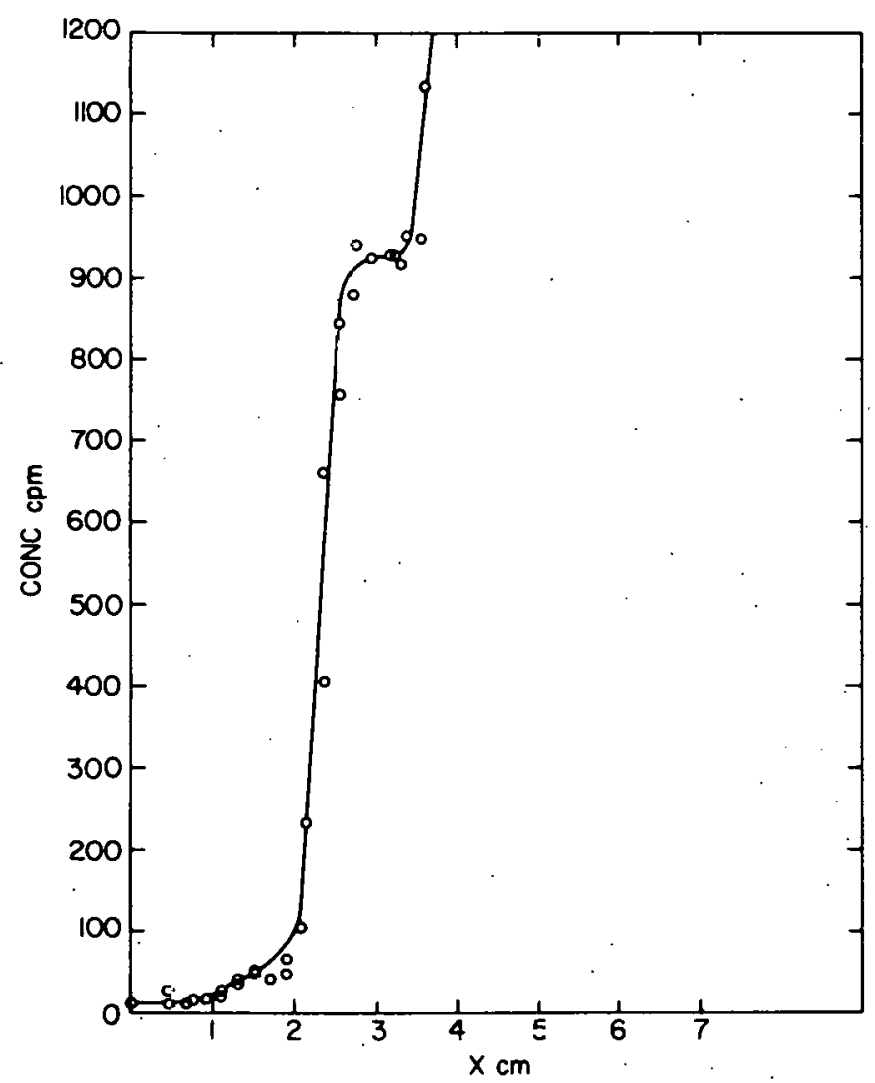

Figure 14a. Concentration profile of 0.1 wt \% C alloy after. $1 . \mathrm{hr}$. electrotransport at $1553^{\circ} \mathrm{K}$.

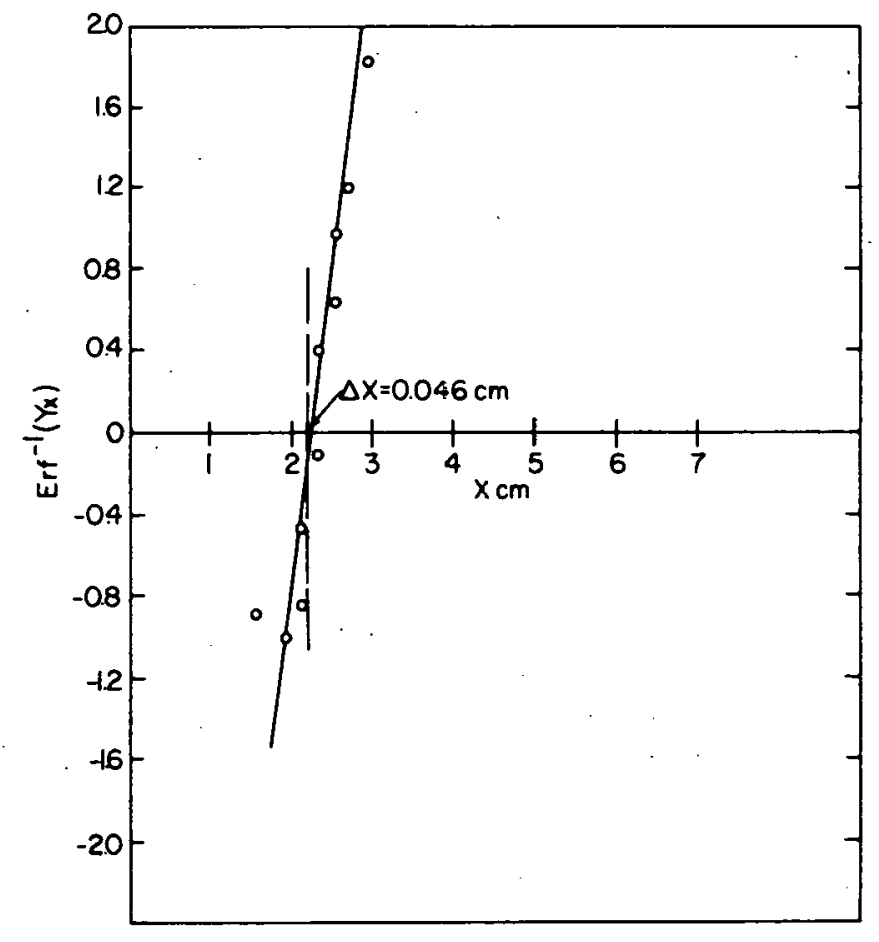

Figure 14b. Inverse error function versus distance plot for the 0.1 wt $\% \mathrm{C}$ alloy used for the $1 \mathrm{hr}$.

electrotransport at $1553^{\circ} \mathrm{K}$. 


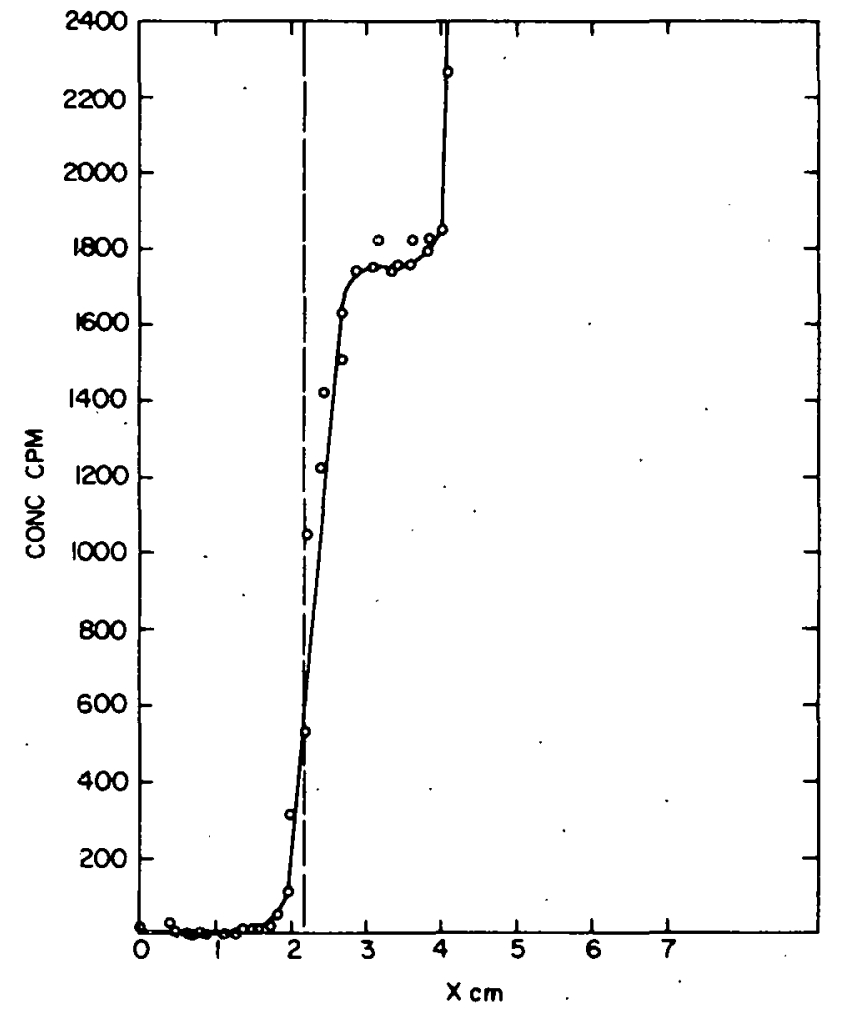

Figure 15a. Concentration profile of the 0.2 wt \% C alloy after 96 hrs. at $1220^{\circ} \mathrm{K}$ under argon atmosphere.

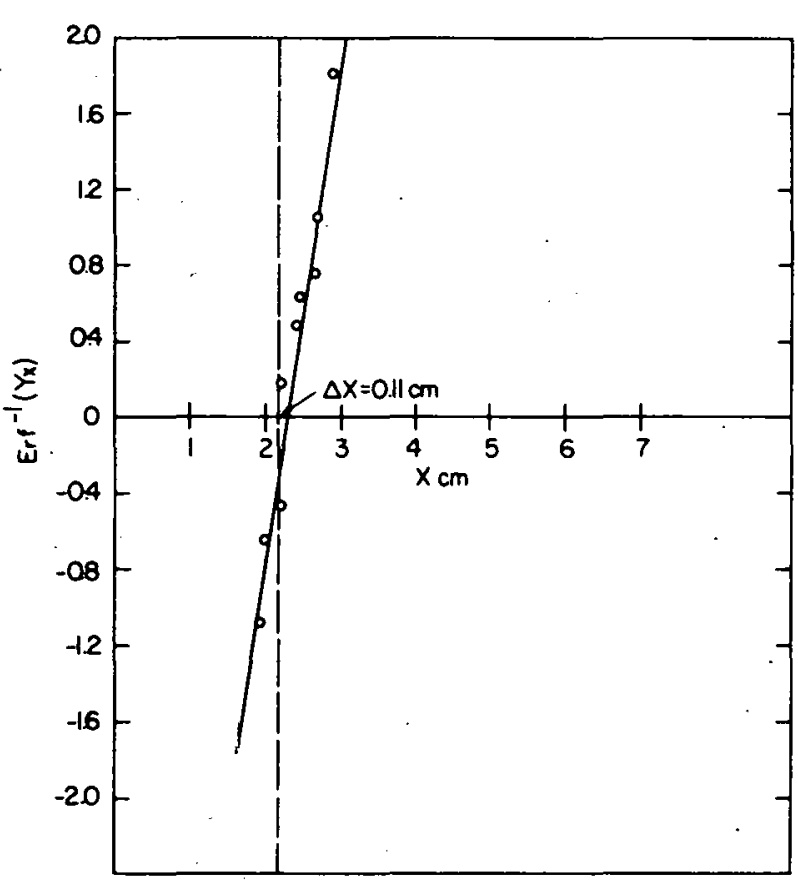

ñ
Figure 15b. Least square fit inverse error function versus distance, plot for 0.2 wt \% C alloy run at $1220^{\circ} \mathrm{K}$ under argon for $96 \mathrm{hrs}$. 


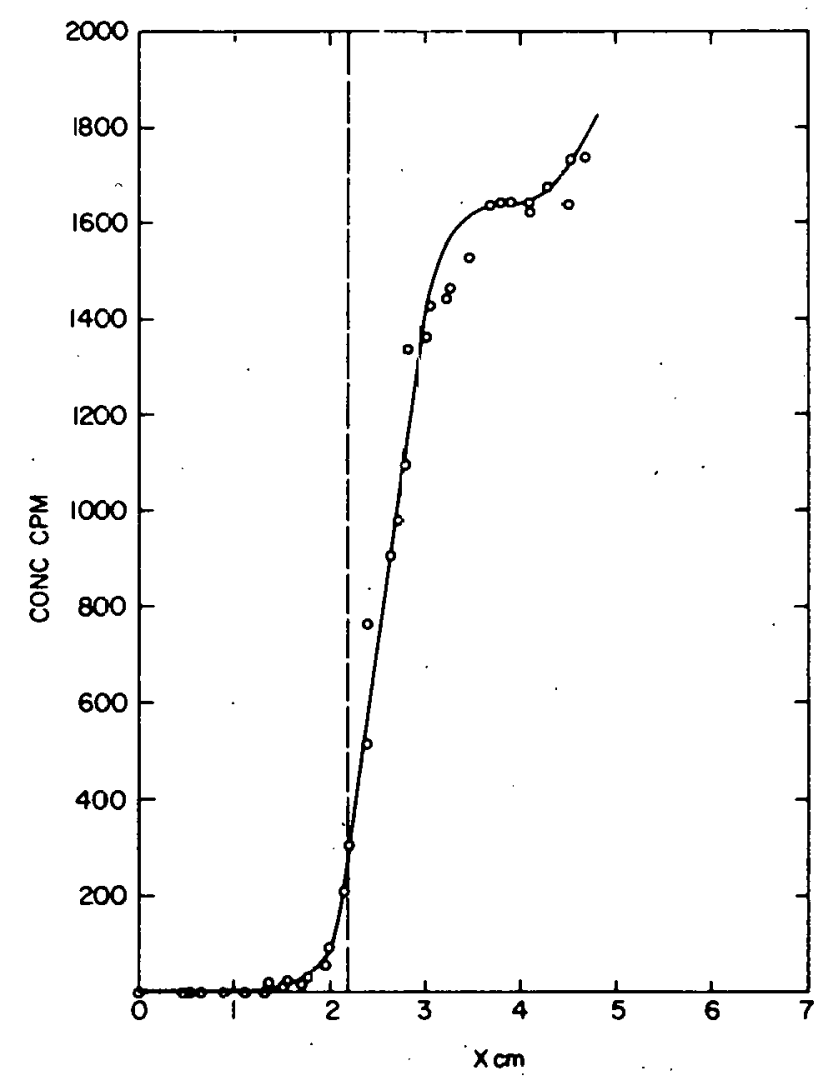

Figure 16a. Concentration profile of 0.2 wt \% C alloy after 93 hrs. electrotransport at $1310^{\circ} \mathrm{K}$.

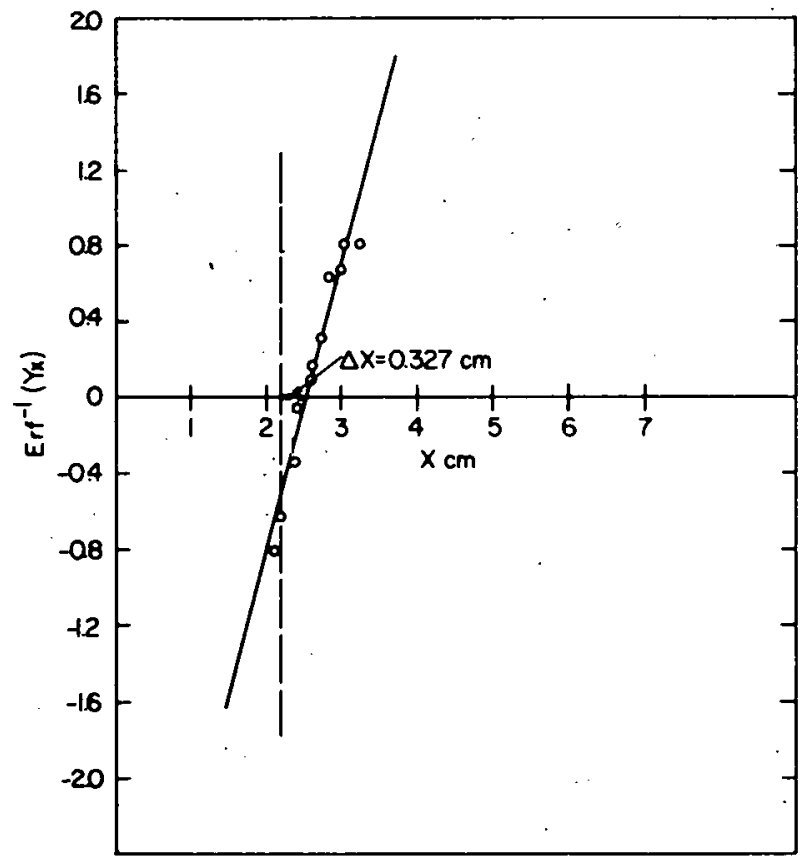

Figure 16b. Inverse error function versus distance plot for 0.2 wt \% C alloy after $93 \mathrm{hrs}$. at $1310^{\circ} \mathrm{K}$. 


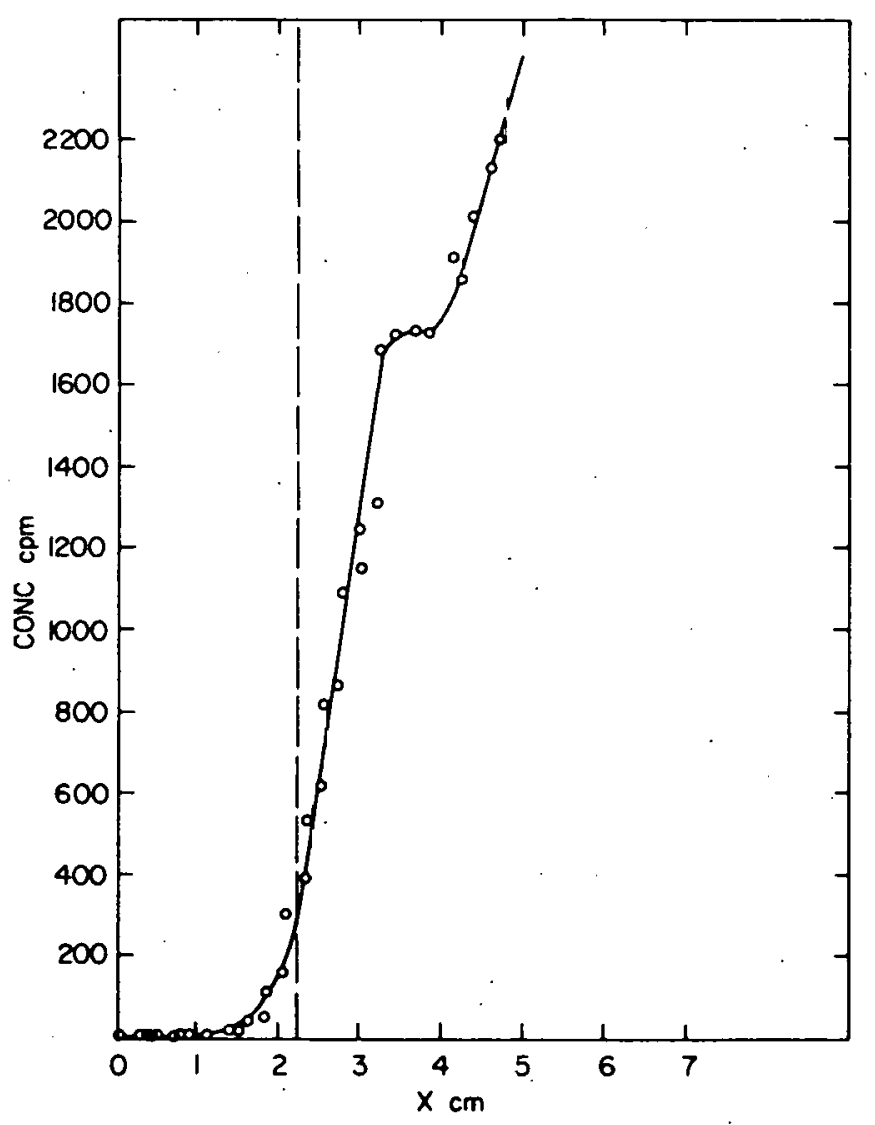

Figure 17a. Concentration versus distance plot for 0.2 wt \% C alloy after $72 \mathrm{hrs}$. at $1367^{\circ} \mathrm{K}$.

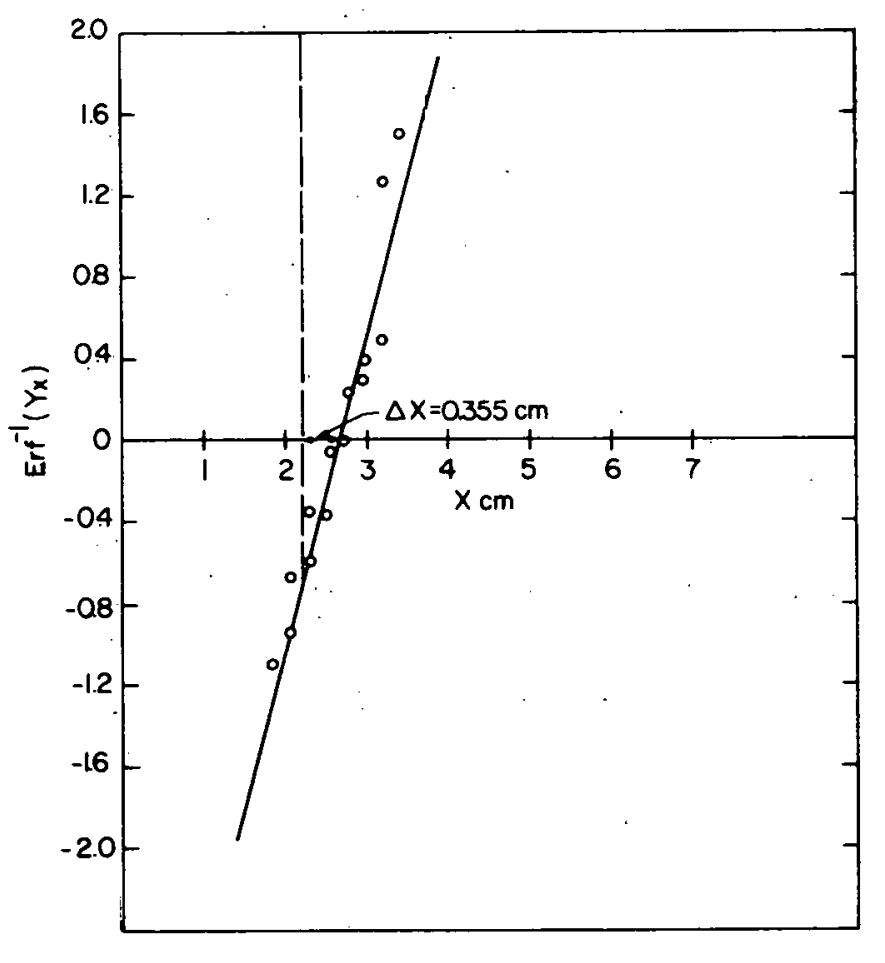

Figure 17b. Least square fit of inverse error function versus distance plot for the 0.2 wt $\% \mathrm{C}$ alloy run at

$1367^{\circ} \mathrm{K}$ for $72 \mathrm{hrs}$. 


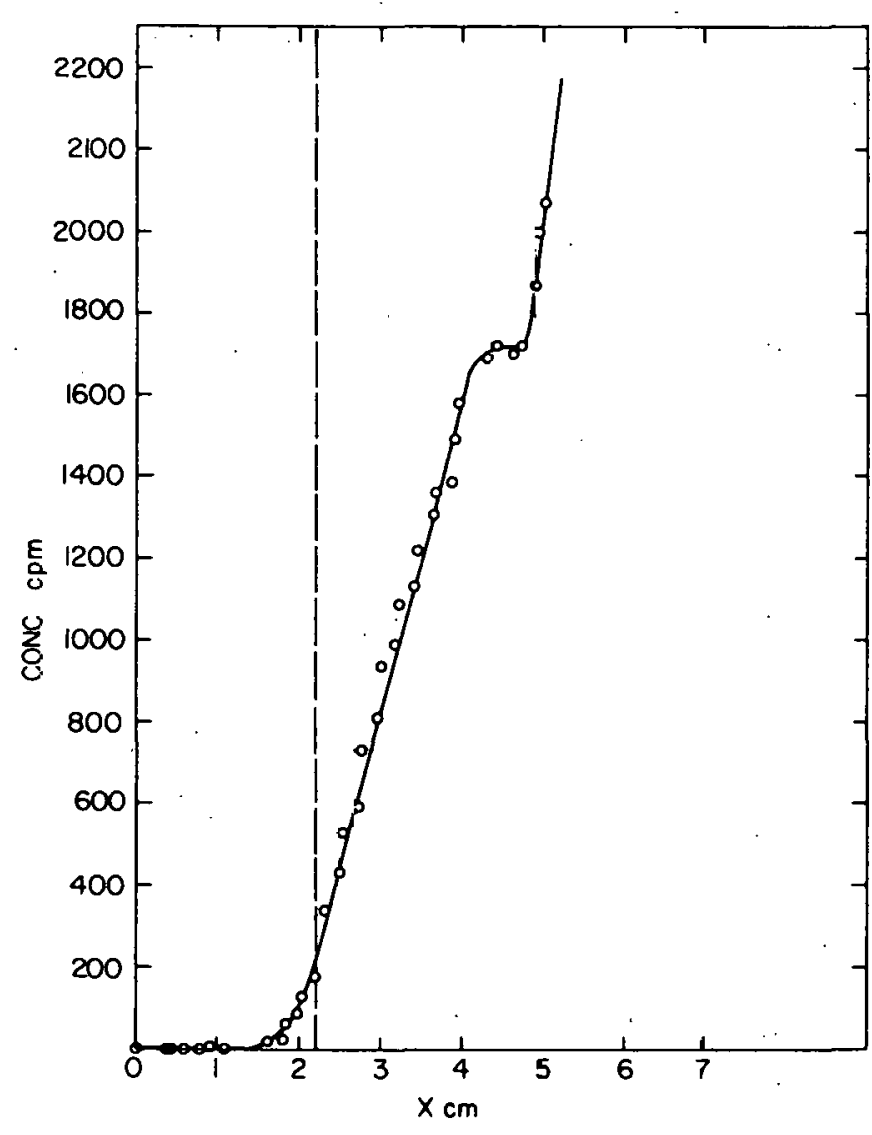

Figure 18a. Concentration profile of the 0.2 wt \% C alloy after $24 \mathrm{hrs}$ electrotransport at $1587^{\circ} \mathrm{K}$.

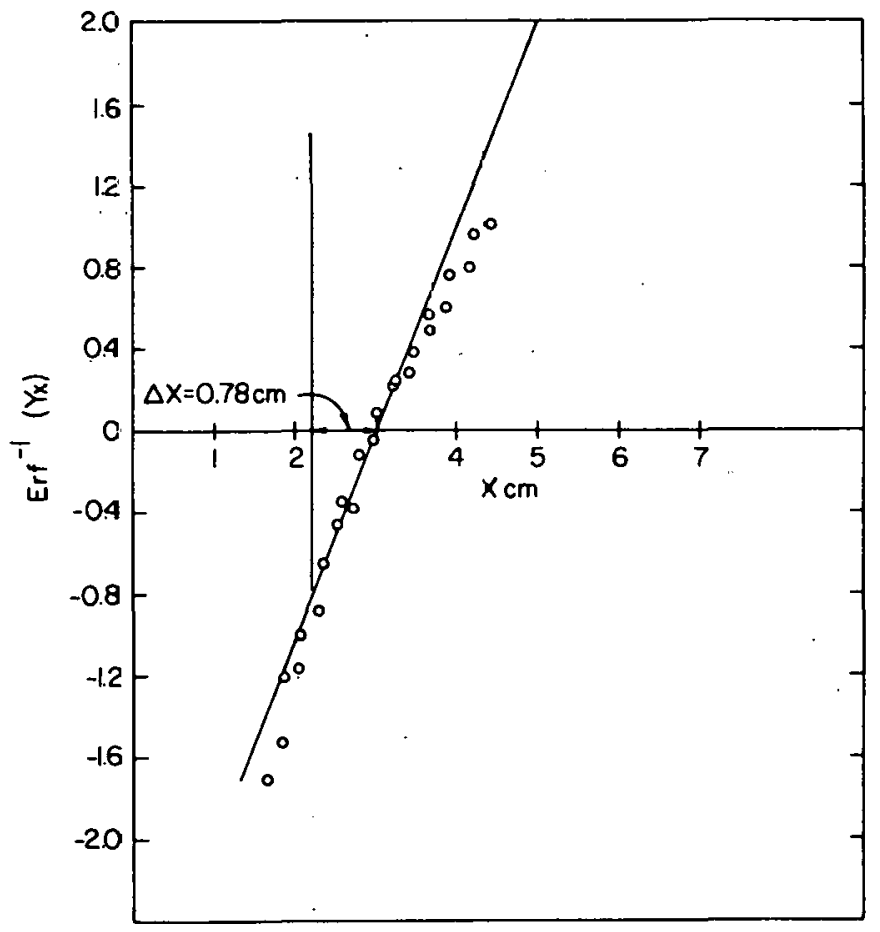

Figure 18b. Inverse error function versus distance plot for the 0.2 wt $\% \mathrm{C}$ alloy held at $1587^{\circ} \mathrm{K}$ for $24 \mathrm{hrs}$. 
Table 4. Electrotransport results

\begin{tabular}{|c|c|c|c|c|}
\hline Ailoy No. & Temperature & $\rho \times 10^{-6} \mathrm{ohm} / \mathrm{cm}$ & $\mathrm{j}=\frac{\mathrm{i}}{\mathrm{A}} \mathrm{amp} / \mathrm{cm}^{-2}$ & $\mathrm{E} \frac{\text { volt }}{\mathrm{cm}}$ \\
\hline & 1217 & 118.50 & 738.64 & 0.08697 \\
\hline (2) & 1313 & 120.20 & 757.58 & 0.09072 \\
\hline \multirow[t]{3}{*}{0.1 wt $\% \mathrm{C}$} & 1361 & 121.25 & 864.89 & 0.09114 \\
\hline & 1580 & 122.50 & 994.32 & 0.12305 \\
\hline & 1220 & 117.75 & 729.88 & 0.08649 \\
\hline (3) & 1310 & 119.75 & 766.37 & 0.09212 \\
\hline \multirow{2}{*}{0.2 wt $\% c$} & 1367 & 120.30 & 848.48 & 0.10288 \\
\hline & 1580 & 123.75 & 957.97 & 0.11735 \\
\hline
\end{tabular}




\begin{tabular}{|c|c|c|c|c|}
\hline $\operatorname{tx} 10^{4} \mathrm{sec}$ & $\mathrm{V} \times 10^{-7} \mathrm{~cm} / \mathrm{sec}^{-}$ & $\mathrm{U} \times 10^{-6} \frac{\mathrm{cm}^{2}}{\mathrm{sec} / \mathrm{volt}}$ & $D \times 10^{-8} \mathrm{~cm}^{2} / \mathrm{sec}^{-1}$ & $\mathrm{z}^{*}$ \\
\hline 40.14 & 3.24 & 3.73 & 9.97 & 3.93 \\
\hline 17.28 & 23.15 & -- & -- & --- \\
\hline 2.88 & 15.63 & 15.24 & 54.25 & 3.71 \\
\hline 0.36 & 127.78 & 112.87 & 432.00 & 3.27 \\
\hline 34.56 & 3.18 & 3.68 & 9.92 & 3.90 \\
\hline 33.48 & 9.77 & 10.61 & 33.20 & 3.61 \\
\hline 25.92 & 13.71 & 1.3 .32 & 44.10 & 3.56 \\
\hline 8.64 & 90.28 & 76.93 & 298.00 & 3.64 \\
\hline
\end{tabular}


also contains both the experimental conditions and the values of the other electrotransport parameters $\mathrm{D}$ and $\mathrm{z}^{*}$.

The diffusivity follows an Arhenius-type relation, $D=D_{0} \exp \left(\frac{-Q}{R T}\right)$. A plot of $\ln D$ versus $\frac{1}{T}$ for the alloys is shown in Fig. 19. For the 0.1 wt $\% \mathrm{C}$ alloy the diffusivity equation is of the form $D(0.1$ wt $\% C)=0.99 \exp \left(\frac{-38.9}{R T}\right)$ and for the 0.2 wt $\% c$ alloy, $D(0.2$ wt $\% C)=0.45 \exp \left(\frac{-37.2}{\mathrm{RT}}\right)$ was obtained. Each set of the data points for both alloys agrees within the limits of precision of the measurements. A least square fit on the data of the two alloys yields an Arhenius equation of the form $D$ (combined) $=0.70 \exp \left(\frac{-38.2}{R T}\right)$. For 0.1 wt $\% \mathrm{C}$ in $\mathrm{Fe}-40$ wt \% $\mathrm{Ni}$ alloy Smith (44) reports diffusivity equation of the form $D=0.20 \exp \left(\frac{33.4}{R T}\right)$. Smith obtained his data from decarburization experiments. Our results indicate slightly higher $D_{0}$ and $Q$ values. The differences in the data points between our 0.1 and 0.2 wt $\% \mathrm{C}$ alloys are within the limits of precision of the measurements. A plot of $\ln \frac{v_{i} T}{j}$ versus $\frac{1}{T}$ is shown in Fig. 20. The linearity of this plot as predicted by the theory in Eq. 24 is obvious. The theory also predicts a similarity between the activation energies obtained from both the slope of this plot and from the diffusion data. The slope of the best straight line through the data gives an activation energy of $\mathrm{Q}=-37.7 \mathrm{kcal} / \mathrm{mol}$ which is in good agreement with the activation energy $Q=-38.2 \mathrm{kcal} / \mathrm{mol}$ obtained from the combined diffusivity data. The activation energy obtained from the $\left(l_{n} \frac{v_{i} T}{j}\right)$ versus $\frac{1}{T}$ plot is about 1.3 percent lower than the diffusion data. The slightly lower value is 


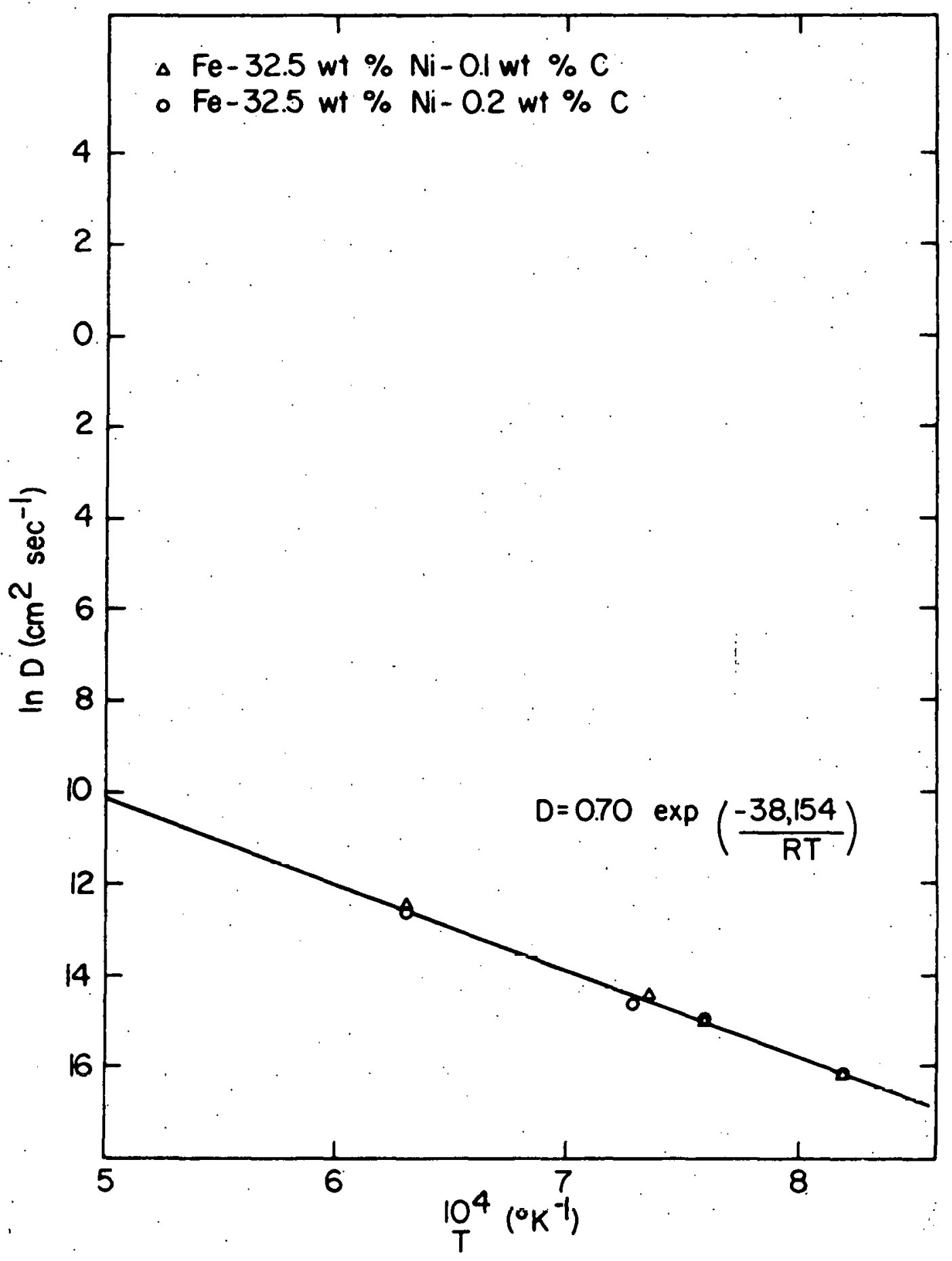

Figure 19. Least squares plot of $\ln D$ versus $\frac{1}{T}$ for the 0.1 and
0.2 wt $\% \mathrm{C}$ alloys. 


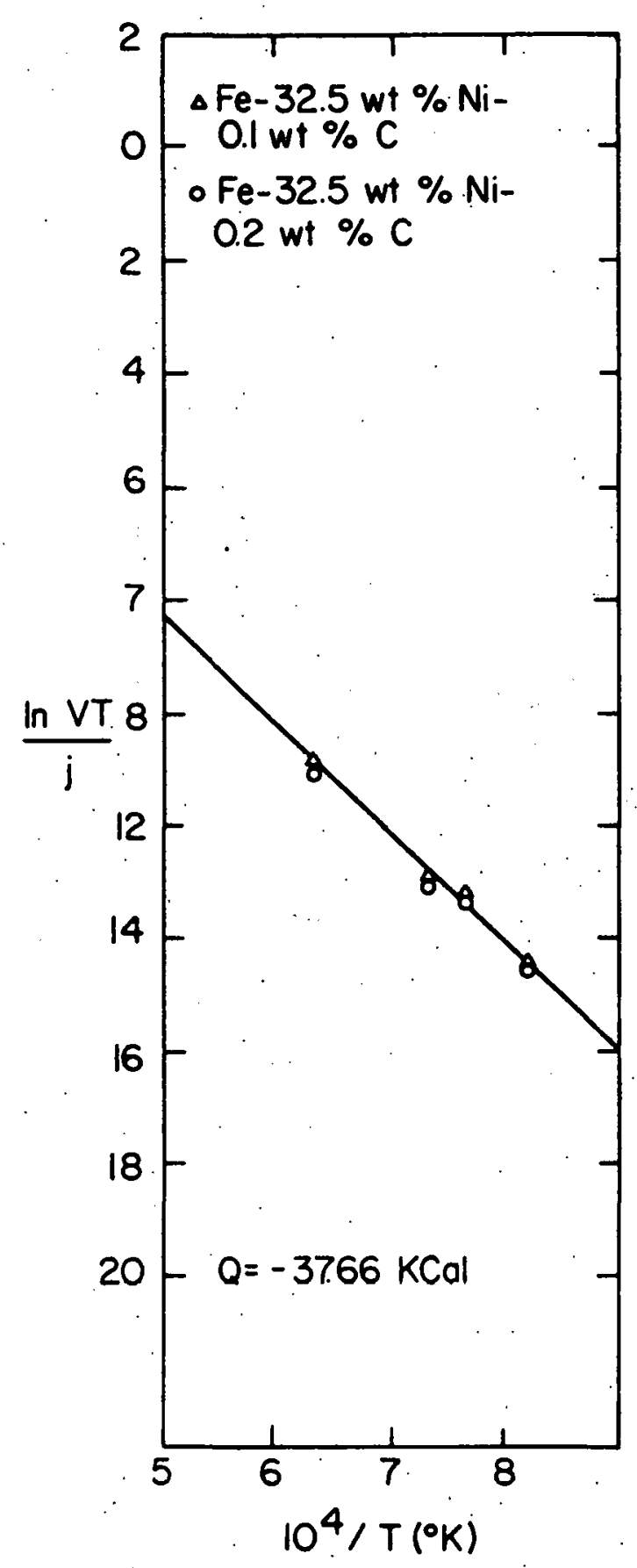

Figure 20. A least squares plot of $\ln \frac{v_{i} T}{j}$ as a function of the
reciprocal of temperature. 
consistent with theory if $\mathrm{z}^{*}$ is independent of temperature as was assumed in Eq. 22 .

The displacement of the weld interface of the electrotransport specimens as indicated by both the concentration-distance and the error function plots indicate that the effective valence $z^{*}$ is positive. The values of $z^{*}$ obtained in this study range from 3.4 to 4.0 . At a temperature of $1220^{\circ} \mathrm{K}, \mathrm{Z}^{*}$ values of 4.0 and 3.9 were obtained for the 0.1 and 0.2 wt \% C alloys. The $z^{*}$ values are in excellent agreement with the value obtained by Nakajima and Hirano (45) who reported 3.9 for an alloy of $\mathrm{Fe}-4$ wt $\% \mathrm{Ni}-0.6$ wt \% C at a temperature of $1233^{\circ} \mathrm{K}$. In the same work Nakajima and Hirano reported that neither the addition of nickel nor chromium of a few percent has an effect on $z^{*}$.

In general, electromigration is due to two "driving forces"; (1) the coulombic force resulting from the applied field and (2) the wind (friction) force exerted on the ion by the electron carriers of the large current that passes through the sample. The sum of both effects is reflected by an "effective charge" $z^{*}$. Verhoeven and Hucke (19) suggest that if the observed value of $\mathrm{Z}^{\text {t }}$ was in fact a joint effect of a coulombic and a friction force, then $z^{*}$ would vary with composition, and perhaps with temperature (46-49). The variation in the $\mathrm{z}^{*}$ values observed for the two alloy compositions seem to be within the 1 imits of experimental errors; but the variation of $z^{*}$ with temperature (Fig. 21) indicates a decrease in $\mathrm{z}^{*}$ with increasing temperature. 


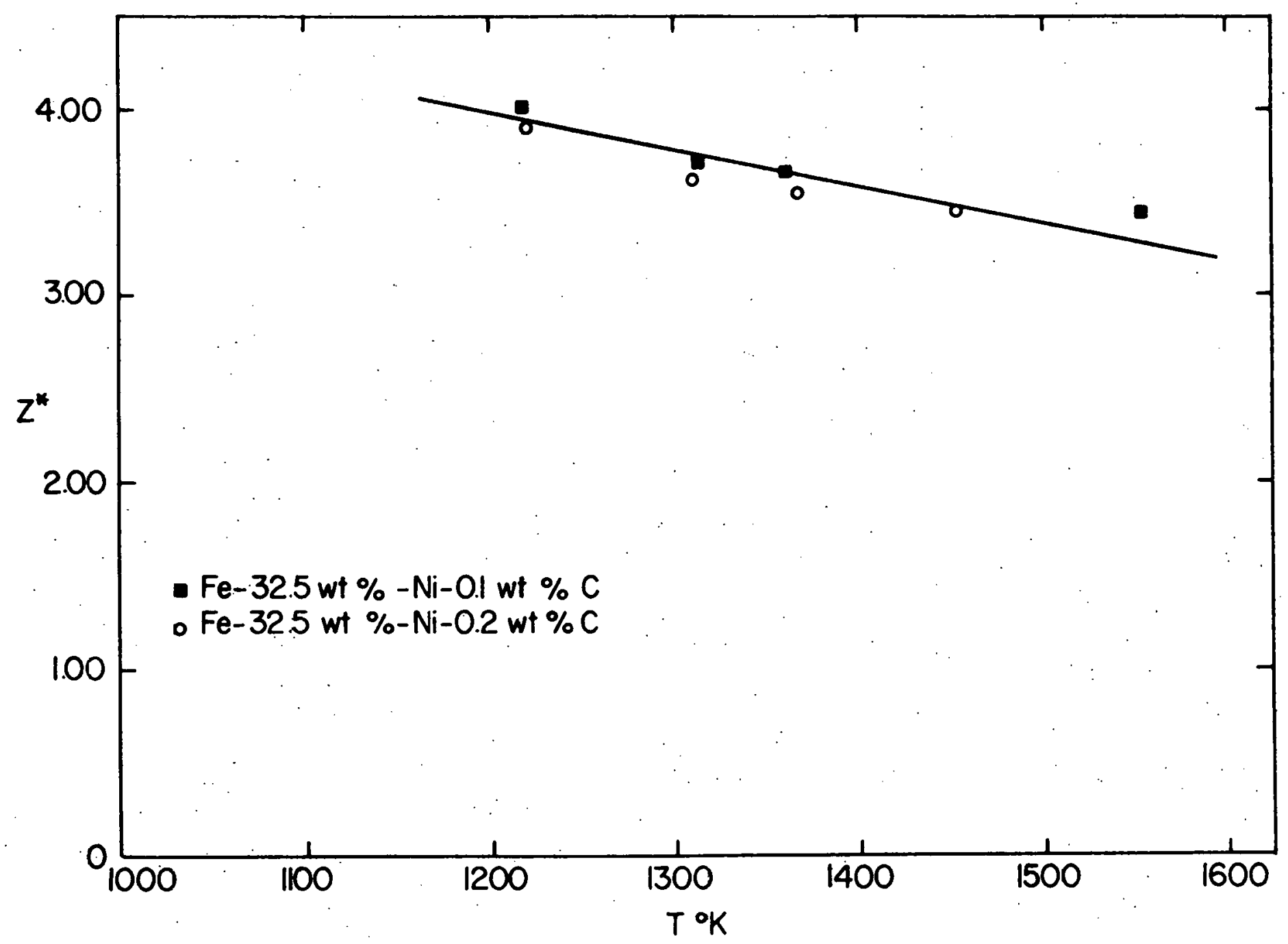

Figure 21. The variation of $\mathrm{z}^{\boldsymbol{x}}$ as a function of temperature. 
From Eq. 4 an effective valence can be defined as the expression $z^{*}=\left[z_{i}-\frac{\delta_{i}}{e^{2} \rho}\right]$. A plot of $z^{*}$ vs $\frac{1}{\rho}$ as a function of temperature should therefore yield a straight line, if the true valence $z_{i}$ and the coefficient of friction $\delta_{i}$ are constant. In Fig. 22 such a plot is shown. The best straight line through the data indicates a good agreement with the theory.

Thermotransport and Determination of $Q^{*}$

The results of the thermotransport studies are summarized in

Table 5. In the one-phase alloys (非3, 非 6 and 非) containing $0.22,0.33$ and 0.37 wt \% C respectively, all of the solute migration is toward the hotter region of the specimen. Both concentration and temperature are plotted as a function of distance for the 0.22 wt \% C alloy in Fig. 23. A similar plot for the 0.37 wt $\% \mathrm{C}$ alloy is shown in Fig. 24 . The data points for the extreme ends of the specimens were omitted in determining $Q^{*}$ partly due to the greater uncertainty in the temperature and also in the actual carbon content because of the presence of the $\mathrm{Cu}$ adapter in those regions of the rods. Plots of $\ln c$ versus $\frac{1}{T}$ are shown in Figs. 25 and 26 for the two alloys. The heats of transport were determined from the slopes of the $\ln c$ versus $\frac{1}{T}$ plots. Both slopes yield a $Q^{*}$ value of $-2.8 \mathrm{kcal} / \mathrm{mol}$ indicating that there is no measurable concentration dependence of $Q^{*}$. Duplicate runs made of the 0.22 wt $\% \mathrm{C}$ specimen gave $Q^{*}$ values of -2.8 and $-2.4 \mathrm{kcal} / \mathrm{mol}$ which gives some indication of the reproducibility of the data. 


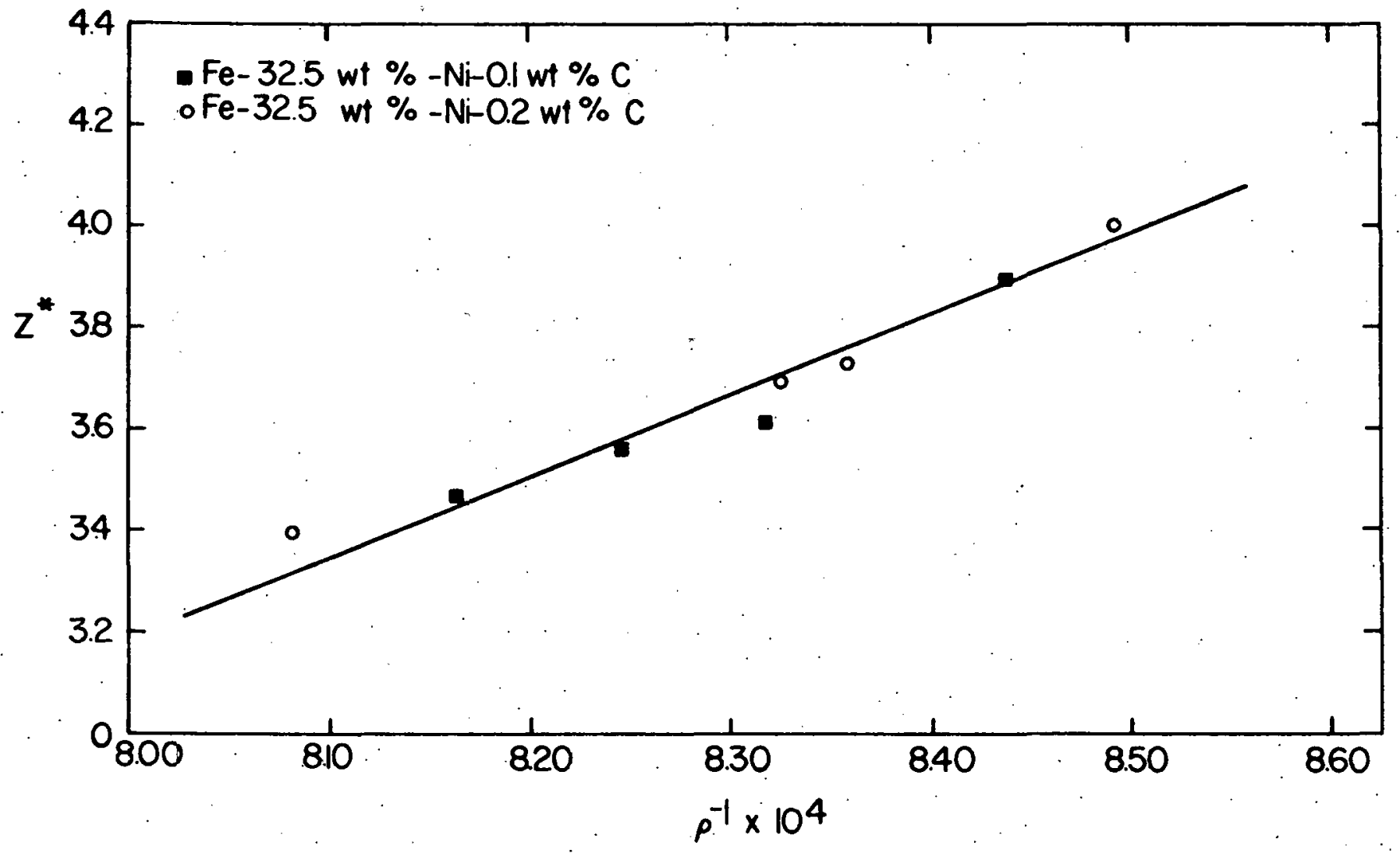

Figure 22. $z^{*}$ versus $\frac{1}{\rho}$ for the two electrotransport alloys. 
Table 5. Summary of thermotransport data

\begin{tabular}{|c|c|c|c|c|}
\hline $\begin{array}{c}\text { Alloy } \\
\text { no. }\end{array}$ & $\begin{array}{l}\text { Co/initial wt } \\
\% \mathrm{C} \text { in the alloy } \\
(\mathrm{Fe}-32.5 \text { wt } \% \mathrm{Ni})\end{array}$ & Atmosphere & $\begin{array}{l}\text { Cm (mean composi- } \\
\text { tion wt } \% \mathrm{C} \text { as } \\
\text { analyzed after run) }\end{array}$ & Phase \\
\hline 3 & 0.22 & Argon & 0.22 & $\gamma(\mathrm{Fe}-\mathrm{Ni})$ \\
\hline $3^{a}$ & 0.22 & Argon & 0.22 & $Y(\mathrm{Fe}-\mathrm{Ni})$ \\
\hline 6 & 0.33 & Vacuum & $0.52 ?$ & $?$ \\
\hline 7 & 0.37 & Argon & 0.27 & $\gamma(\mathrm{Fe}-\mathrm{Ni})$ \\
\hline $7 \mathrm{~A}$ & 0.41 & Vacuum & 0.52 & $\gamma+\mathrm{Fe}_{3} \mathrm{C}$ \\
\hline 9 & 0.62 & Argon & 0.45 & $\gamma+\mathrm{Fe}_{3} \mathrm{C}$ \\
\hline 9 & 0.62 & Vacuum & 0.68 & $\gamma+\mathrm{Fe}_{3} \mathrm{C}$ \\
\hline \multirow[t]{4}{*}{$9^{a}$} & 0.62 & Vacuum & 0.73 & $Y+\mathrm{Fe}_{3} \mathrm{C}$ \\
\hline & 0.02 & $?$ & - & $\gamma-F \dot{e}$ \\
\hline & 0.33 & $?$ & - & $\gamma-F e$ \\
\hline & Not available & $?$ & - & $\mathrm{Ni}$ \\
\hline
\end{tabular}

${ }_{\text {Duplicate. }}$ 


\begin{tabular}{|c|c|c|c|}
\hline $\begin{array}{c}\text { Temperature } \\
\text { range } \\
o / C\end{array}$ & $\begin{array}{c}Q^{*} \\
\mathrm{kcal} / \mathrm{mol}\end{array}$ & $\begin{array}{l}\text { Build up } \\
\text { at cold end }\end{array}$ & Reference \\
\hline $1.330-1490$ & -2.4 & None & This study \\
\hline $1325-1500$ & -2.8 & None & This study \\
\hline $1200-1410$ & -5.0 & None & This study \\
\hline $1212-1440$ & -2.8 & None & This study \\
\hline $1200-1410$ & -4.9 & Large positive & This study \\
\hline $1212-1440$ & -2.9 & Large positive & This study \\
\hline $1210-1440$ & -3.0 & Large positive & This study \\
\hline $1220-1450$ & -3.0 & Large positive & This study \\
\hline $1210-1229$ & $-2.2 \pm 1.5$ & - & 34 \\
\hline $1193-1214$ & $-2.0 \pm 1.5$ & - & 34 \\
\hline $1241-1273$ & $-1.6+1.0$ & - & 36 \\
\hline
\end{tabular}




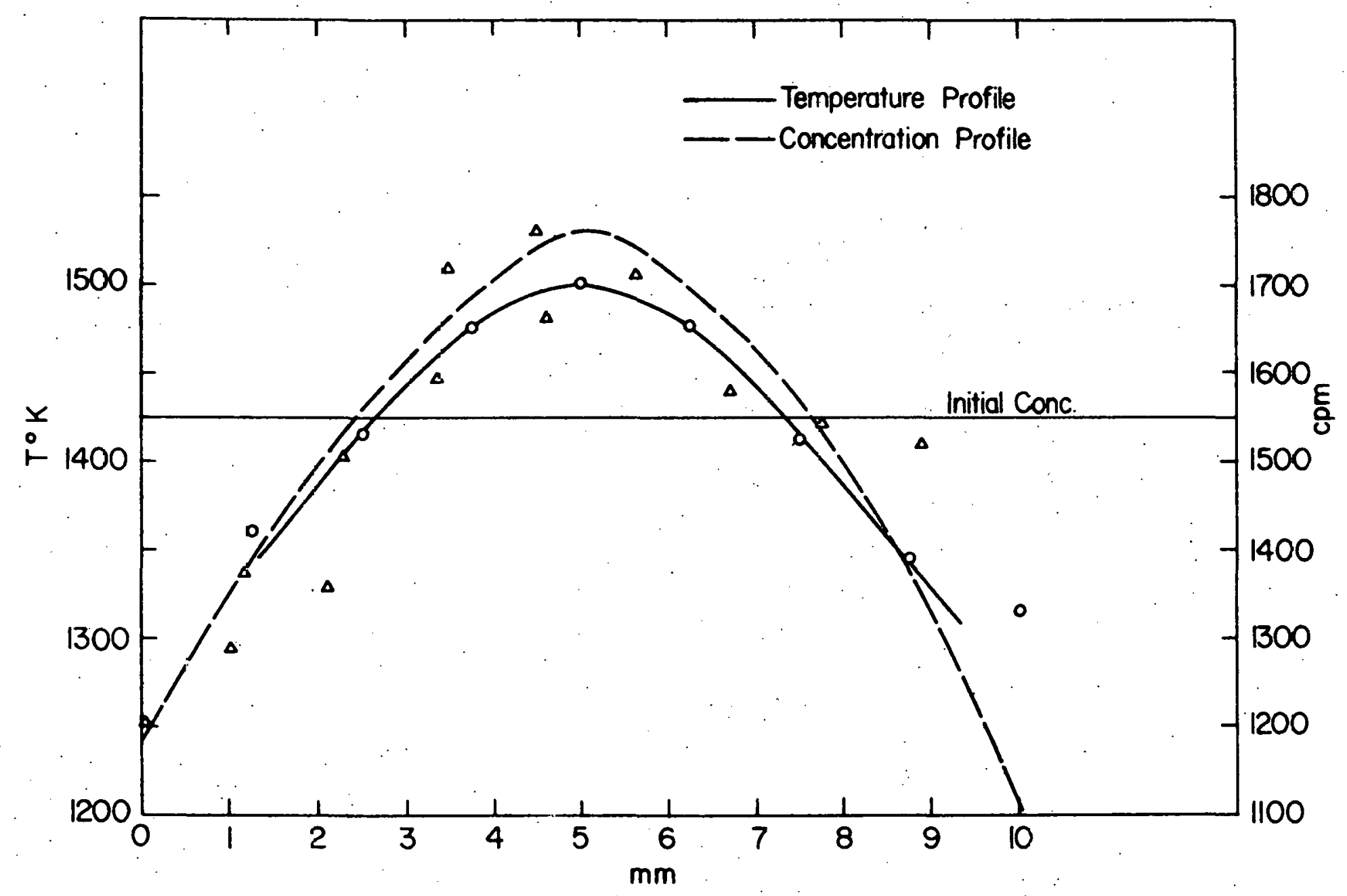

Figure 23. Plot of temperature and concentration as a function of distance. 


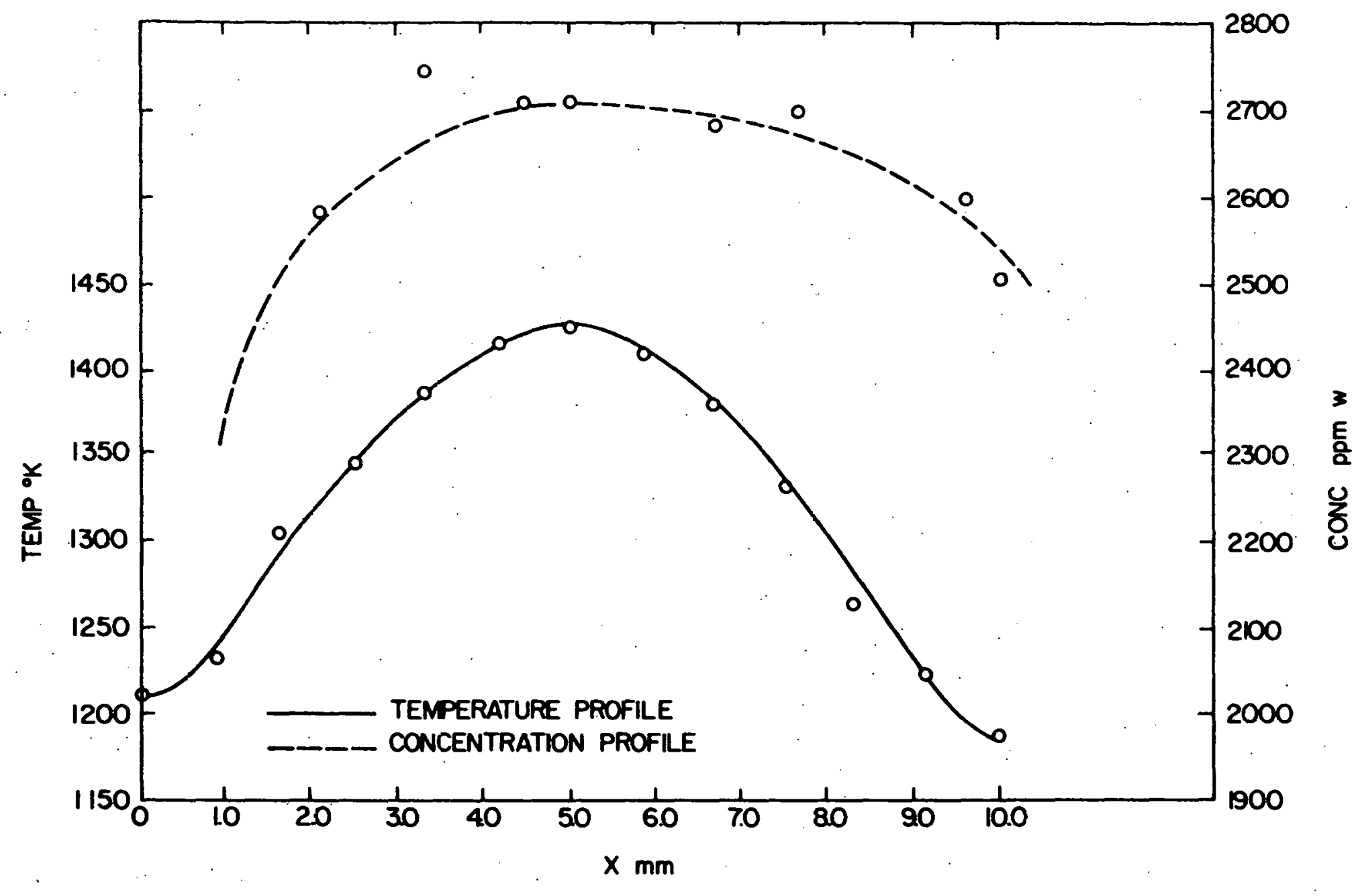

Figure 24. Concentration and temperature versus distance plot for the initially 0.37 wt \% C alloy. 


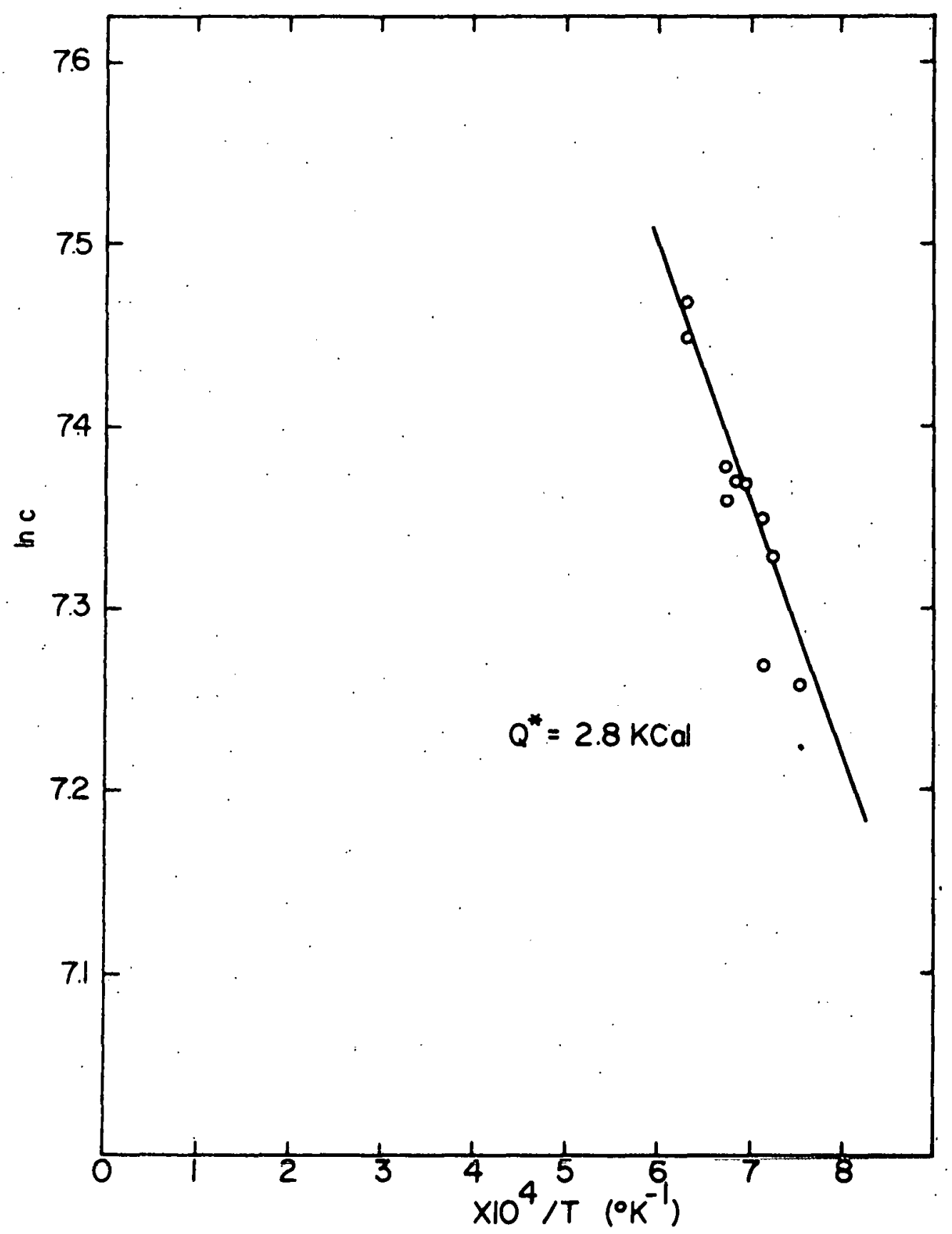
Figure 25. Least squares plot of $\ln c$ as a function of $\frac{1}{T}$ for the
0.22 wt $\%$ C alloy. 


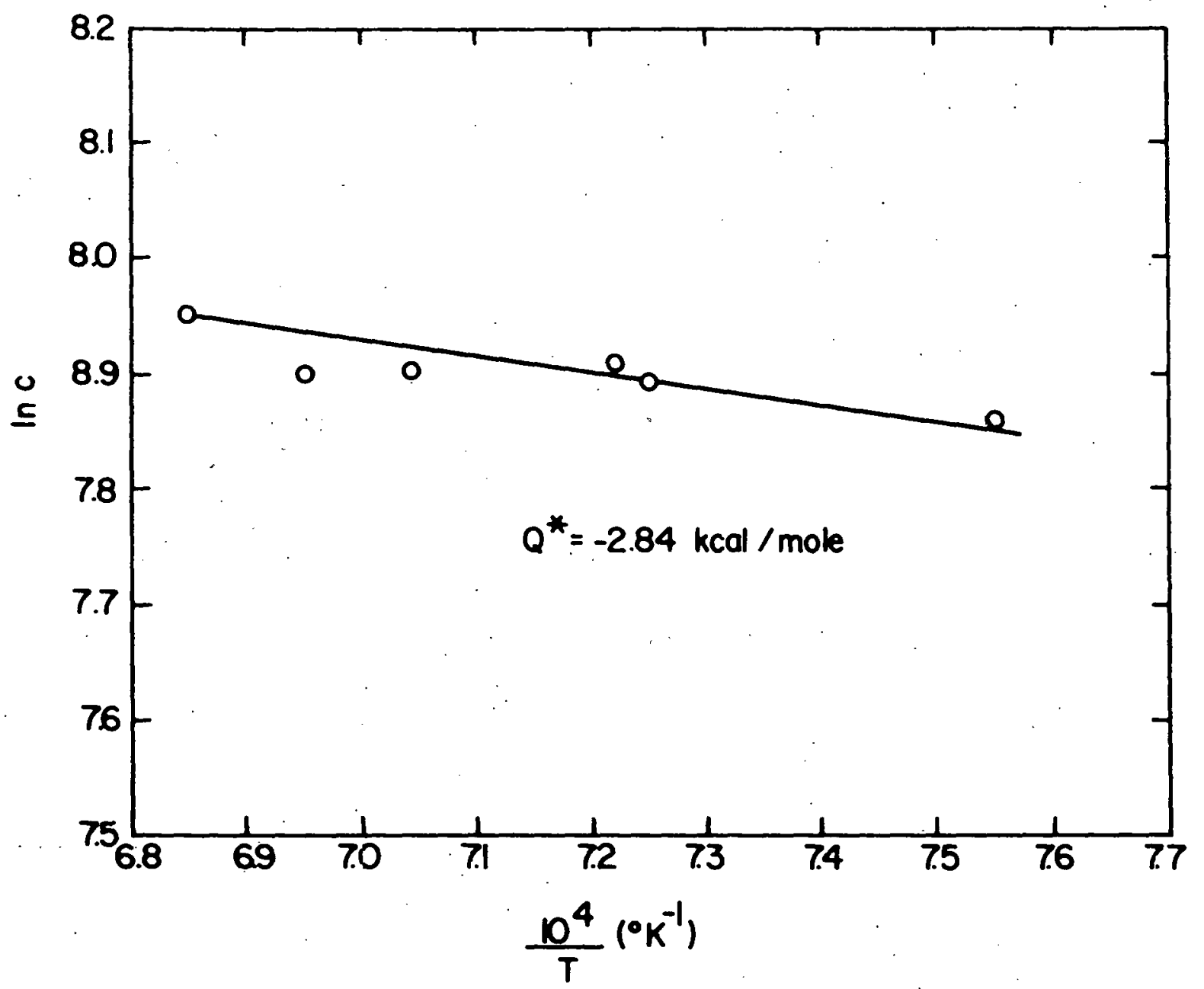

Figure 26. Least squares plot of $\ln \mathrm{c}$ as a function of $\frac{1}{\mathrm{~T}}$ for the initially 0.37 wt $\% \mathrm{C}$ alloy. 
A large carbon loss was encountered in the nominal 0.37 wt \% C alloy run, in which losses reduced the mean concentration to an estimated 0.27 wt \% C. This problem is discussed in greater detail in the next section.

In the two-phase alloys (非 7 and $\# 9$ ) containing 0.41 and 0.62 wt $\% \mathrm{C}$ a combination of two transport processes seems to be involved. Concentration versus distance plots along with the temperature profiles obtained for 0.62 wt \% C specimen are shown for two different runs in Figs. 27 and 28. The first run was made under a partial pressure $(13 \mathrm{~mm})$ of purified argon for a period of 50 hours. A mass balance shows a significant decrease in carbon from that of the original concentration. The presence of carbon-like material on the surface of the copper adapters was noted. Another run was made on the same alloy in a vacuum of $\sim 10^{-7}$ torr at a lower temperature range for a shorter period of time, in an attempt to avoid the carbon loss. The time of run was 17 hours which was still long enough to achieve $90 \%$ steady state. A small carbon gain from 0.62 to 0.68 wt \% C was encountered during this run. A duplicate run, except for a longer time ( 55 hours), gave almost identical results.

A comparison of Figs. 23, 24, 27 and 28 indicates that solute migration is towards the hotter regions of the sample when the alloy is in a one-phase condition. This is indicated by the concentration peaks in the region of the temperature maxima in all runs. Minima observed in the concentration-distance plots for the 0.62 wt $\% \mathrm{C}$ alloy occur at about $1050^{\circ} \mathrm{C}(1323 \mathrm{~K})$. An alloy of this composition crosses the solvus 


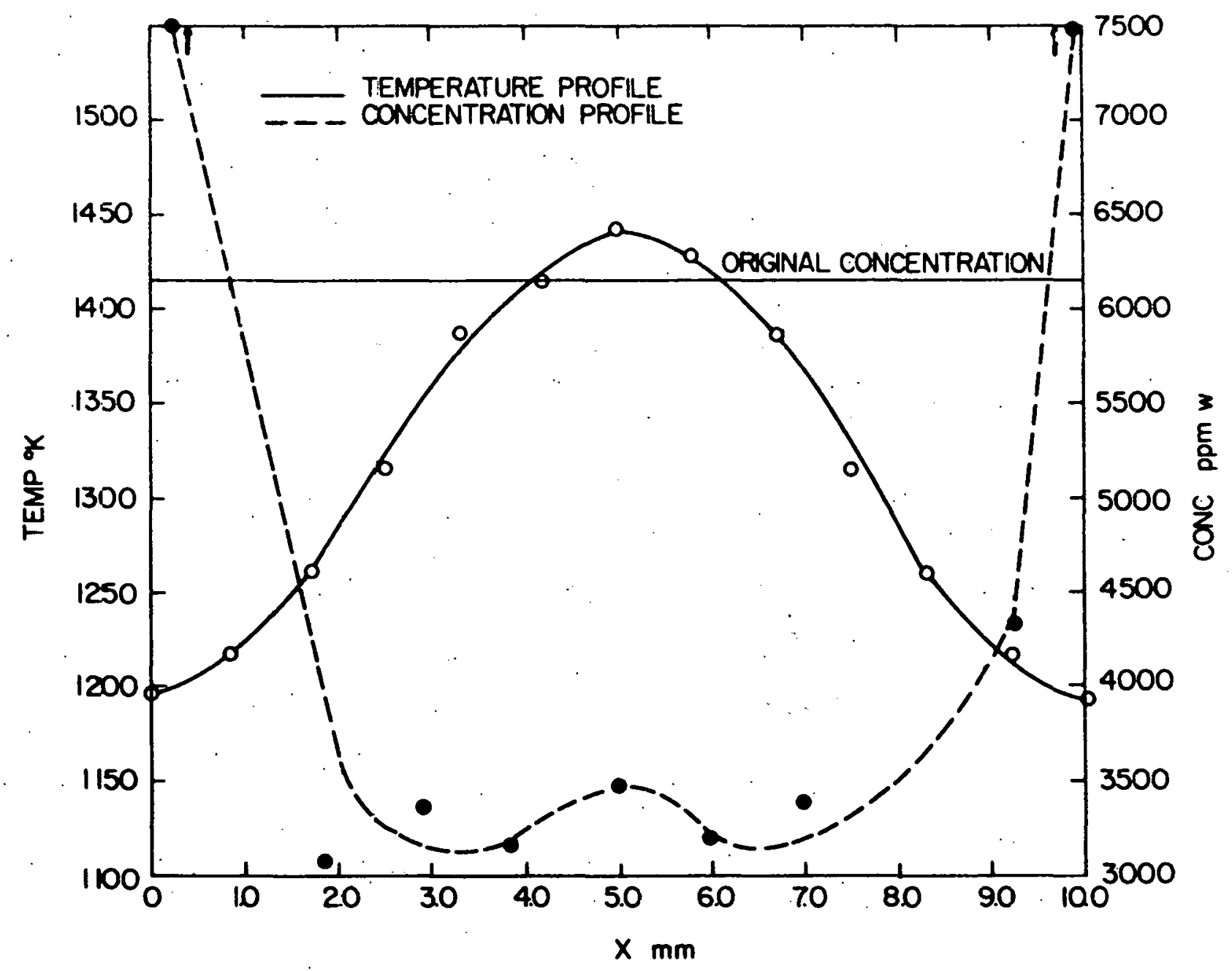

Figure 27. A plot of concentration and temperature as a function of distance for the first 0.62 wt \% C alloy. 


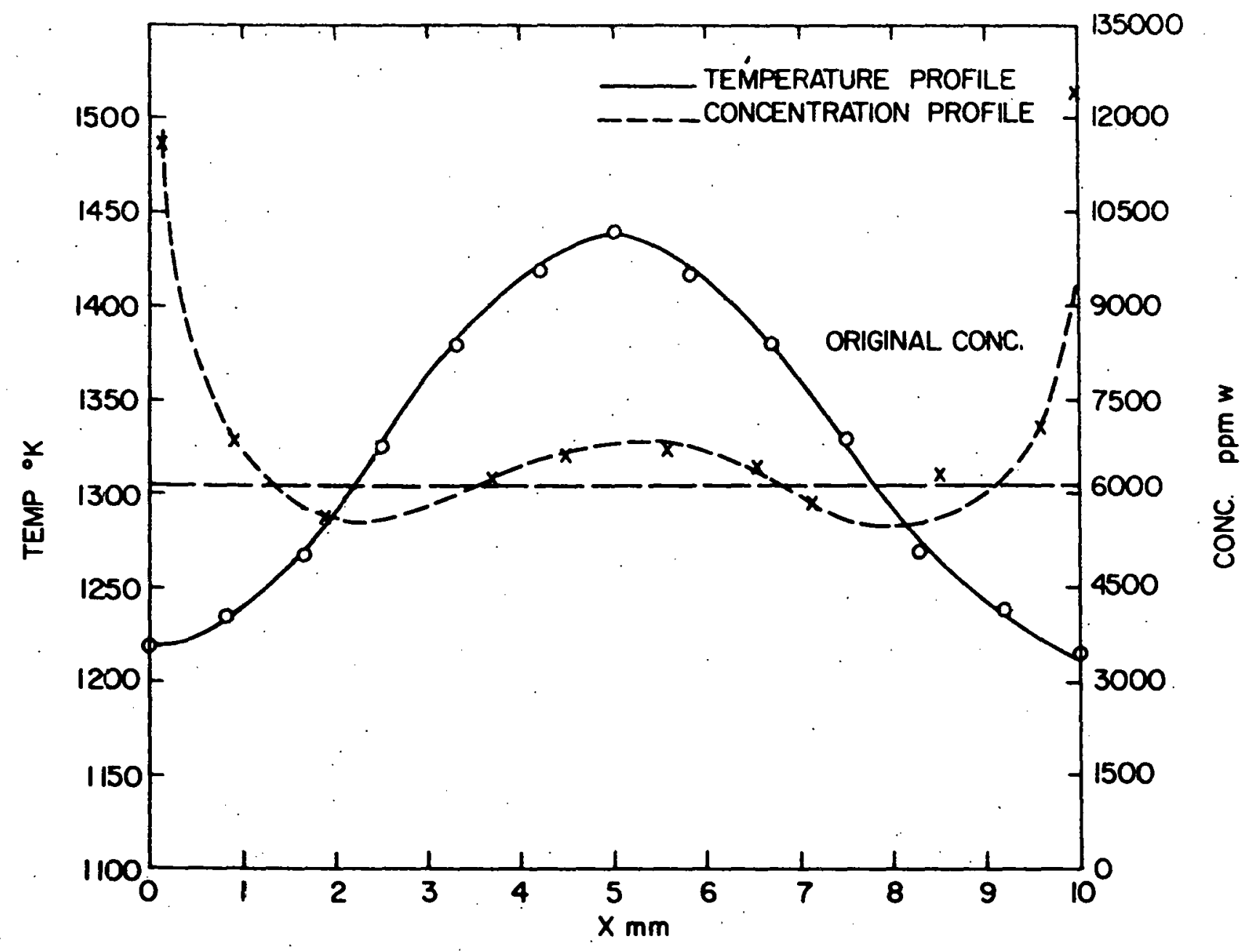

สั.

Figure 28. Plot of concentration and temperature as a function of distance for the second 0.62 wt \% C alloy. 
boundary (Fig. 4) somewhere between 1040 and $1060^{\circ} \mathrm{C}$. The 0.41 twophase alloy likewise shows minima at $1020^{\circ} \mathrm{C}$ which correspond to the solvus crossing temperature for that alloy. The agreement between the minimum temperature and the temperature at which the alloy crosses the solvus suggests a possible relationship between the two, although it may be merely coincidental. Portions of the specimens below the minima temperatures 1 ie in the $Y+\mathrm{Fe}_{3} \mathrm{C}$ region according to the solubility plot (Fig. 4). The net migration in these lower temperature portions of the specimen is toward the colder ends. Apparently two phenomena are involved, thermotransport which is apparent from alloys in the one-phase condition and a larger mass transport effect toward the older regions, referred to herein as a pseudo heat of transport, for alloys in the two-phase condition. Plots of $\ln c$ versus $\frac{1}{T}$ for. the 0.62 wt $\% \mathrm{C}$ alloy (Figs. 29 and 30 ) show anomalously large "Q*" values of 14.1 and $28.6 \mathrm{kcal} / \mathrm{mol}$ respectively for that portion of the alloy lying in the two-phase region at the lower temperature. The actual significance of this larger transport effect is not yet understood. As a result the effect is referred to in Table 5 only as a carbon 'build up at cold end'. Some of the experimental specimens (Table 5) were observed to have picked up carbon during the run, so the weighted mean carbon concentration $C_{m}$ is also noted in the table. 


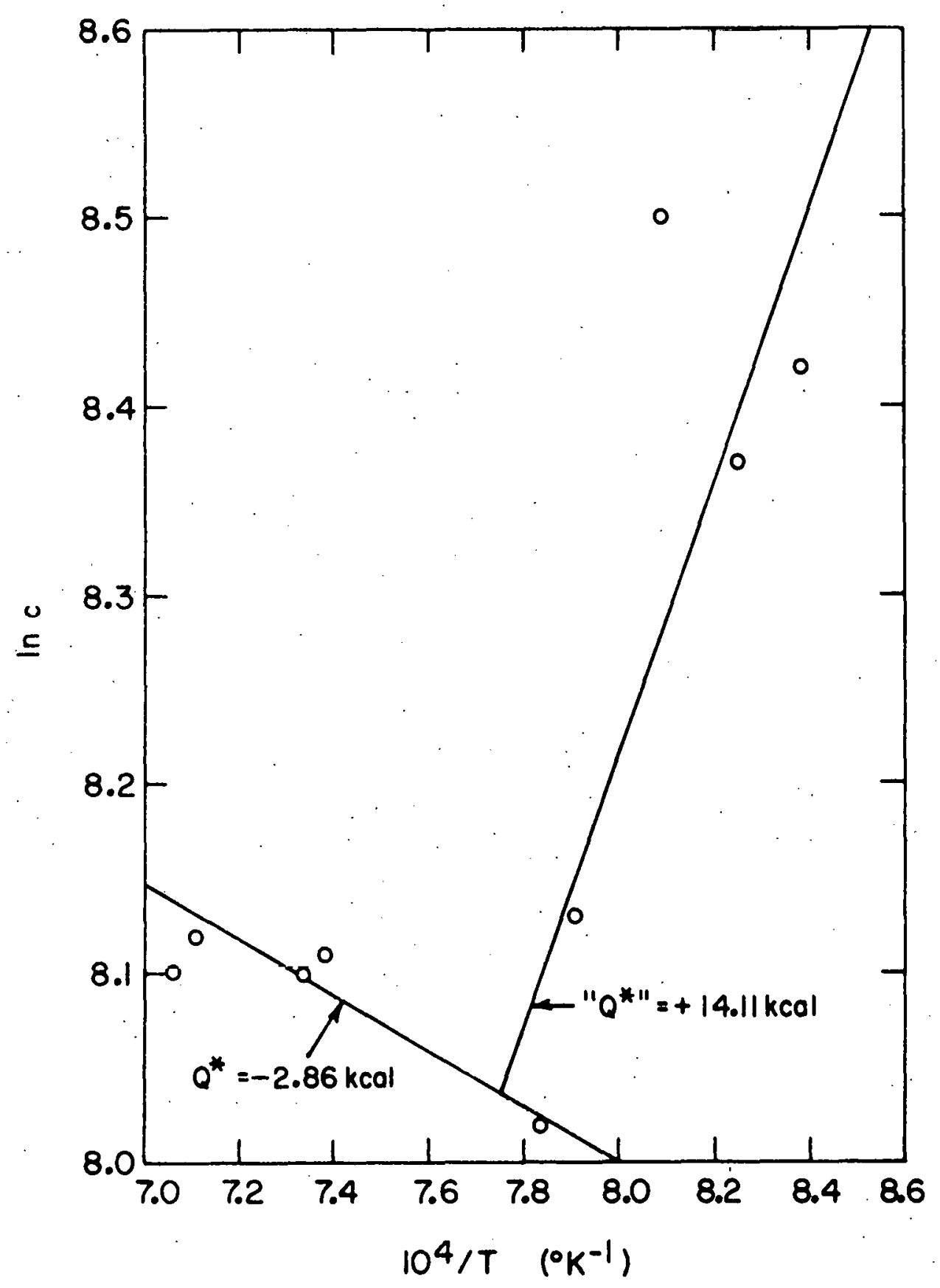
Figure 29. Least squares plot of $\ln c$ versus $\frac{1}{T}$ for the first
0.62 wt $\%$ C $3110 y$. 


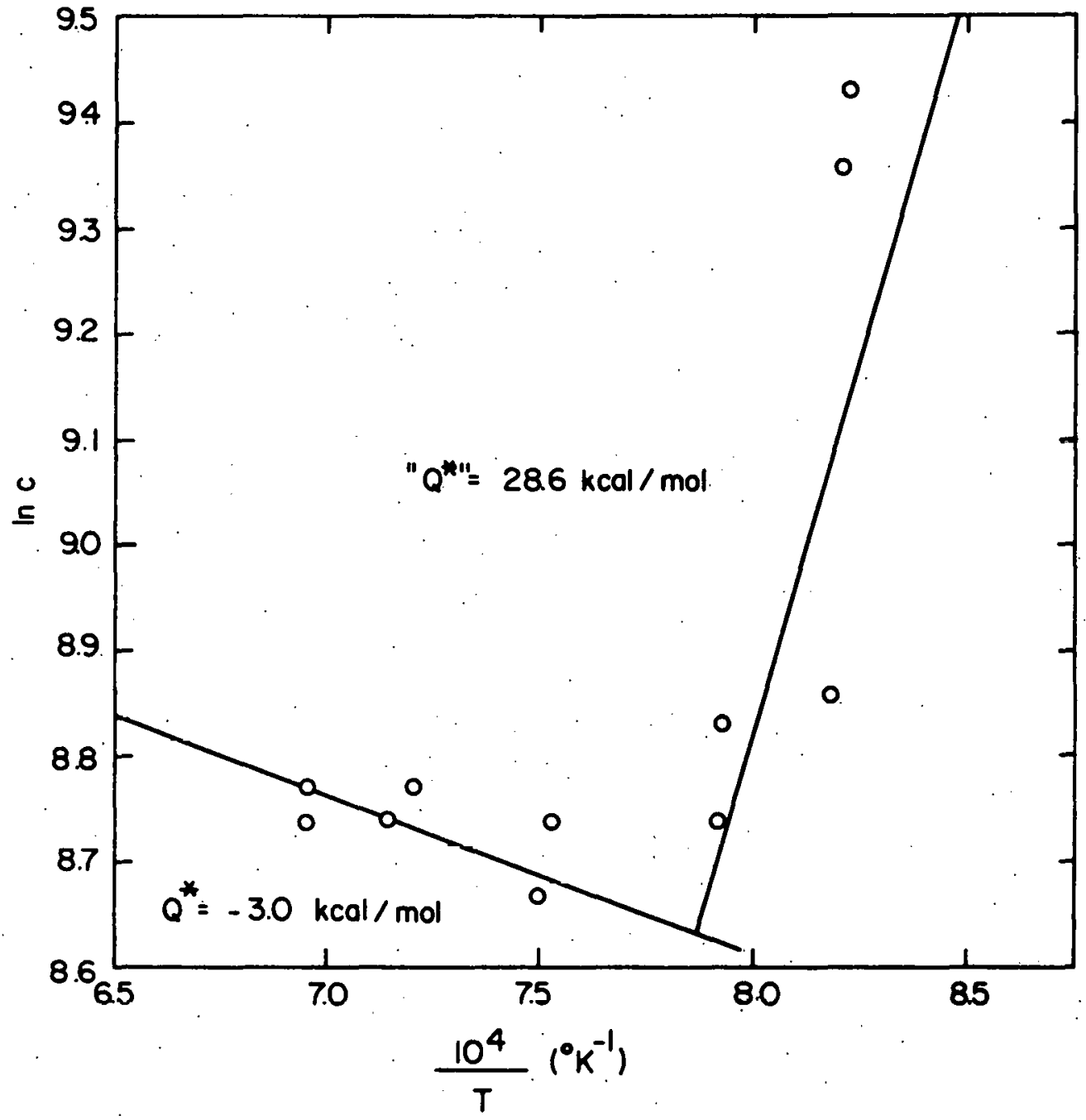

Figure 30. Least squares $p l o t$ of $\ln c$ as a function of $\frac{1}{T}$ for the
second 0.62 wt $\% C$ alloy. 


\section{DISCUSSION}

The problems associated with the decarburization of the Fe-Ni alloys particularly in the long-term electro- and thermotransport runs are described in detail in the Appendix. Unless the upper plateau for the electrotransport run coincides with the initial concentration of the doped portion of the rod, the results of an experiment were discarded. This condition has been met for a11 of the electrotransport data included in this dissertation.

In a thermotransport run if no solute is either lost or gained there should be a mass balance between solute displaced from one region to another. If one assumes equal sized samples, the area between the curve above the initial concentration line should be equal to the area on the opposite side bounded by the initial concentration line and the concentration profile resulting from the experiment. This condition was met in the duplicate runs for the 0.22 wt \% C alloy and for the second run of the 0.62 wt $\% \mathrm{C}$ alloy. In the 0.37 wt $\% \mathrm{C}$ run and the first 0.62 wt \% C run carbon losses were encountered that brought the mean carbon concentration to approximately 0.27 and 0.45 wt $\% \mathrm{C}$ respectively. Apparently, a near steady state condition was attained for both of these runs at these lower mean concentrations inasmuch as the $Q^{*}$ values are in agreement with those obtained for those runs made under no carbon loss conditions. It is pertinent to add that whereas the mass balance in Fig. 28 may appear to indicate significant carbon gain, in actuality this is not so. The large areas under the curve at the 
ends of the sample are exaggerated by the relatively smaller size analytical sample pieces. If a correction for this is taken into account, the carbon pickup is relatively small.

Our average value of $Q^{*}$ is $-2.8 \mathrm{kcal} / \mathrm{mol}$ which is in the same range as Shewmon's (34) values of $Q^{*}=-2.0$ and $-2.2 \mathrm{kcal} / \mathrm{mol}$ for 0.02 and 0.33 wt $\% C$ in $\gamma-F e$ respectively (Table 5). Shaw and oates (36) reported a $Q^{*}$ value of $-1.6 \mathrm{kcal} / \mathrm{mol}$ for carbon in $\mathrm{Ni}$. The anomalously high pseudo $Q^{*}$ values obtained for the two-phase alloy deserves further mention. It is speculated that some 1 arge $Q^{*}$ values reported in the literature may be mostly due to this solubility effect than to true thermomigration. Similar anomalous behavior has been observed by Carlson and weins (50) in their recent work on carbon migration in two-phase $\mathrm{V}$ - Ti alloys.

Finally, the problem associated with determining the actual solubility limit of carbon in this system using quenching practices and metallographic work should be mentioned. The major problem is that of the thermodynamically unstable $\mathrm{Fe}_{3} \mathrm{C}$ which is made even more unstable by the graphitizing effect of nickel. This made the interpretation of the microstructure very difficult. Furthermore, it 1s possible that the slow kinetics at low temperatures may make the time to reach equilibrium prohibitively long. 


\section{BIBLIOGRAPHY}

1. K. Nates an and T. F. Kassner, Met. Trans., 4, 2557 (1973).

2. K. J. Irvine, T. Gladman and F. B. Pickering, J. Iron Steel Inst., 207, 1017 (1969).

3. J. E. White and J. W. Freeman, Trans. ASME, J. Eng. Power, $\underline{85}, 119$ (1963).

4. R. Nakagawa and Y. Otoguro, Tetsu-To-Hagane, 47, 1169 (1961).

5. Y. Kawabe, R. Nakagawa and T. Mukoyama, Trans. Iron Steel Inst. Jap., 8, [6], 353 (1968).

6. L. M. T. Hopkin and L. H. Taylor, J. Iron Steel Inst., 205, 17 (i967).

7. P. D. Goode11, T. M. Cullen and J. W. Freeman, Trans. ASME, J. Basic Eng., 89, 517 (1967).

8. P. G. Stone, "High Temperature Properties of Stee1", Proc. Joint Conf. organized by BISRA and ISI, 505 (1966).

9. I. A. Rohrig, Effects of Residual Elements on Properties of Austenitic Stainless Steels, ASTM-STP, 418, 78 (1966).

10. J: K. Y. Hum and N. J. Grant, Trans. ASM, 45, 105 (1953).

11. J. T. Barnby and F. M. Peace, Acta Met., 19, 1351 (1971).

12. R. Stickler and A. Vinckier, Trans. ASM, 54, 362 (1961).

13. H. F. F.hling and M. $\Lambda$. Echeil, Advainces 1n the 'l'echnology of Stainless Steels and Related Alloys, ASTM-STP, 369, 275 (1965).

14. B. Weiss and R. Stickler, Met. Trans., $\underline{3}, 851$ (1972).

15. G. F. Tissinai, J. K. Stanley and C. H. Samans, Trans. ATME, 200, 1259 (1954).

16. H. Bethe, Report of the Cornell Workshops on the Major Issues of a National Energy Research and Development Program; HEDL 7405-117.2, Dec. 1973.

17. H. B. Huntington, "ASM Seminar Book on Diffusion", H. I. Aaronson, Ed., American Society for Metals: Metals Park, Ohio, 1974, p 155. 
18. H. B. Huntington, In "Diffusion in Solids: Recent Developments", A. S. Nowick and J. J. Burton, Eds.; Academic Press: New York, $1974 ;$ p 303.

19. J. D. Verhoeven and E. E. Hucke, Trans. Met. Soc., 227, 1156 (1963).

20. J. N. Pratt and R. G. R. Sellors, Diffusion and Defect Monograph Series, Trans. Tech., Riehen, Switzerland (1973).

21. H. B. Huntington and A. R. Grone, J. Phys. Chem. of Sol., 20, 76 (1961).

22. H. B. Huntington and S. C. Ho, J. Phys. Soc. Japan, $\underline{8}$, Supp 1. H, 202 (1962).

23. V. B. Fiks, Sov. Phys.-Solid State, $\underline{6}, 1251$ (1964).

24. V. B. Fiks, "Proc. Europhys. Conf. Atomic Transport in Solids and Liquids Manstrand", A. Lodding and T. Lagerwa11, Eds., 1970, (Tubingen: Verlag. Z. fur Naturforsch), p 19.

25. C. Bosvieux and J. Friede1, J. Phys. Chem. of Sol., 23, 123 (1962).

26. H. Nakajima and K. Hirano, J. App1. Phys., 48, 1793 (1977).

27. R. S. Sorbe11o, Comments on Solid State Physics, 6 , 117 (1975).

28. A. R. Allwatt and A. V. Chadwick, Chem. Rev., 67, 681 (1967).

29. L. Onsager, Phys. Rev., 37, 405 (1931).

30. S. R. de Groot, "The Thermodynamics of Irreversible Processes", Interscience Publishers, Inc: : New York, 1951.

31. D. O. Rale1gh and A. W. Sommer, J. Chem. Phys., 36, 381 (1962).

32. S. R. de Groot, "L'Effet Soret", North-Holland Publishing Co.: Amsterdam, 1945.

33. K. G. Denbigh, "The Thermodynamics of the Steady State, Methuens' Monographs on Chemical Subject", Methuen and Company Ltd.; London, 1951 .

34. P. Shewmon, Acta Mer., 8, 605 (1960).

35. P. Shewmon, J. Chem. Phys, 29, 1032 (1958).

36. J. G. Shaw and W. A. Oates, Met. Trans., 2, 2127 (1971).

37. E. Soehncheir and E. P1wowarsky, Arch. Eisenhuetten, $\underline{5}, 111$ (1931). 
38. P. Chiotti, Rev. Sci. Inst., 25, 876 (1954).

39. M. Mason and W. Weaver, Phys. Rev., 23, 412 (1924).

40. 0. N. Carlson, F. A. Schmidt and D. T. Peterson, J. Less-Common Metals, 10, 1 (1966).

41. D. J. Fray and J. Chipman, Trans. Met. Soc. ATME, 245, 1143 (1969).

42. L. P. Elliott, In "ASM Metals Handbook", Taylor Lyman, Ed.; American Society for Metals: Metals Park, Ohio, 1973; Vol. 8, p 413.

43a. J. W. Sands, In "ASM Metals Handbook", Taylor Lyman, Ed.; American Society for Metals: Metals Park, Ohio, 1948; p 600.

43b. P. R. Bevington, "Data Reduction and Error Analysis for the Physical Sciences", McGraw-Hill Book Co.: New York, 1969, pp 180-183.

44. R. P. Smith, Trans. Met. Soc., 236, 1224 (1966).

45. H. Nakajima and K. Hirano, Phys. Stat. Sol., (a) 43, K57 (1977).

46. D. F. Kalinovich, Sov. Phys.-Solid State, 3 , 812 (1961).

47. M. J. Bibby, L. C. Hutchinson and W. V. Youdelis, Can. J. Phys., 44, $2375(1966)$.

48. E. A. Falquero and W. V. Youdelis, Can. J. Phys,, 48, 1984 (1970):

49. R. A. Oriani and 0. D. Gonzalez, Trans. TMS-AIME, 239, 1041 (1969).

50. 0. N. Carlson and W. N. Weins, "Thermotransport of Carbon in V-Ti Alloys". To be published.

51. O. Kubaschewski and C. B. Alcock, "Metallurgical Thermochemistry", Pergamon Press: New York, 1979, p 378.

52. R. P. Smith, J. Amer. Chem. Soc, 68, 1163 (1946).

53. U. F. Stein, J. R. Low, Jr., and A. U. Sezbolt, Acta Met., 11, 1253 (1963).

54. K. Natesan and T. F. Kassner, Met. Trans., 4, 2557 (1973). 


\section{ACKNOWLEDGMENTS}

I would like to thank a number of people whose help and encouragement have been invaluable. I owe especial thanks to Professor 0 . N. Carlson for his knowledgeable guidance, diligent encouragement, invaluable suggestions arid critical analysis in many aspects of this study. I also wish to express my appreciation to F. A. Schmidt for his helpful suggestions, to. H. Baker for the metallographic services and to L. Lincoln for his technical assistance in preparing the samples. A special acknowledgment is due to Professor $\mathrm{P}$. Chiotti for his help in the resistivity studies and to G. Austin for the chemical analysis on which the success of the latter thermotransport studies largely depended. I would like to thank Barbara Dubberke very specifically for typing this thesis so well. Finally I would like to thank my wife, Betty, and our sons, Ari and Ndu, for their unparalleled patience, understanding and encouragement. This work was supported by the U.S. Department of Energy, Office of Basic Energy Sciences, Materials Science Division. 
APPENDIX A

This work began with a study of carbon transport in an Fe-17 wt \% Cr ferritic alloy. We. worked with this alloy for a little over a year, within which period we encountered enumerable problems. The initial studies with the ferritic alloy were done in a chamber equipped with only a mechanical pump (Welch Duo-Seal Model 1397B). The best vacuum obtained in that setup was about $1.5 \times 10^{-2}$ torr. Runs were made in the chamber either under vacuum or back-filled with $13 \mathrm{~mm}$ of purified argon. Zirconium wires of $0.04 \mathrm{~cm}$ diameter were used as "getters". The high concentration end of the butt-welded specimen had $100 \mathrm{ppm}$ by wt (1800 cpm) carbon while the other end was essentially carbon free. The maximum carbon solubility at $\sim 950^{\circ} \mathrm{C}$ in this alloy is $\sim 200 \mathrm{ppm}$. An electrotransport run made at $950^{\circ} \mathrm{C}$ for 74 hours in a purified argon atmosphere showed a carbon loss of about $30 \%$ as was indicated by a drop in the carbon concentration of the upper plateau. A duplicate run made in vacuum showed that a lot of metal vapors had condensed on the sight glass and a carbon loss of about $25 \%$. Perhaps, although the inert gas prevented metal vaporization, it contained some impurities that were not gettered. A small amount of hydrogen or oxygen gas in the system could cause carbon losses noted in the range In question. A green oxide film identified as chromium oxide was always observed on the surface of the specimens after heating in either vacuum or inert gas. 
We, therefore, modified our vacuum system by including an oil diffusion pump in the setup. A vacuum of $10^{-7}$ torr was readily reached. Subsequent runs in vacuum yielded carbon losses of about $15 \%$ on the average which was still too much. We finally concluded that the low solubility of carbon in the $\mathrm{Fe}-\mathrm{Cr}$ alloy was responsible for much of the problem, since the loss of even a small amount of carbon represented the loss of a significant fraction of the total present. Since we were unable to purify the atmosphere so it was completely free of hydrogen and oxygen, the problem of some decarburization was always present. It was decided, therefore, to study an alloy with a much higher carbon solubility on the assumption that the loss of a few ppm of carbon could be tolerated since it would not represent a significant fraction of the total carbon present. On the other hand, we felt that this alloy should also be of practical interest. Based on the reasons discussed in the Introduction, the Fe-Ni-C system was chosen.

Electrotransport runs were made on the Fe-32.5 wt $\% \mathrm{Ni}$ alloy in a vacuum of $10^{-7}$ torr at 850 and $950^{\circ} \mathrm{C}$ and in a purified argon atmosphere at $1100^{\circ} \mathrm{C}$ for 150,74 and 6 hours, respectively. The temperatures quoted include emissivity and sight glass corrections. The two vacuum runs showed losses of about 2 - $3 \%$. After the new equipment had been thoroughly out-gassed, the carbon loss during the vacuum runs dropped to less than $1 \%$ which was considered acceptable, Similar runs made under a purified argon atmosphere indicated 5 - 15\% decarburization depending on the temperature and length of time of the 
experiment; these are too high for good electrotransport results. The electrotransport samples were supported at each end by a tantalum adapter. The adapters used in each argon atmosphere run had significant beta counts after heating. The adapter at the doped end of the sample gave beta counts of $1350 \mathrm{cpm}$, whereas the "pure" end adapter gave $511 \mathrm{cpm}$. It is suggested that traces of $\mathrm{H}_{2}$ in the inert gas could contribute to the decarburization as follows.

$$
\begin{array}{ll}
\mathrm{CH}_{4}=\mathrm{C}+2 \mathrm{H}_{2} & \Delta \mathrm{G}^{\mathrm{O}}{ }_{1200}=-10,000(51) \\
\mathrm{Ta}+\mathrm{C}=\mathrm{TaC} & \Delta \mathrm{G}^{\mathrm{O}}{ }_{1200}=-34,300(51) \\
\mathrm{Ta}+\mathrm{CH}_{4}=\mathrm{TaC}+2 \mathrm{H}_{2} & \Delta \mathrm{G}^{\circ}{ }_{1200}=-44,300
\end{array}
$$

From Eq. 55 the mass action law expression is

$$
K_{(52)}=\frac{\mathrm{P}^{2} \mathrm{H}_{2}}{\mathrm{PCH}_{4}} \text {. }
$$

Since this system at least approaches equilibrium, we can write

$$
\begin{aligned}
\Delta G_{(55)}^{\circ}=-R T \ln K= & -44,300 \\
-\ln K= & \frac{-44,300}{4.575(1200)}=-8.07
\end{aligned}
$$

As an indication, let us assume $\mathrm{PH}_{2}$ in the system as $10^{-5}$ atm. Then, 


$$
\begin{aligned}
& \log \frac{\mathrm{P}_{2}^{2} \mathrm{H}_{2}}{\mathrm{PCH}_{4}}=\log \frac{10^{-10}}{\mathrm{PCH}_{4}}=8.07 \\
& \frac{10^{-10}}{\mathrm{PCH}_{4}}=1.175 \times 10^{8} \text { and } \mathrm{PCH}_{4}=8.5 \times 10^{-19} \text { atm. }
\end{aligned}
$$

Using Smith's (52) relation, we deduce that at equilibrium, the amount of carbon left in the alloy would be

$$
\text { (0.9) } \frac{\mathrm{PCH}_{4}}{\mathrm{P}^{2} \mathrm{H}_{2}}=\text { wt } \% \mathrm{C}
$$

and wt $\% \mathrm{C}=(0.9)\left(8.5 \times 10^{-9}\right)=7.7 \times 10^{-9} \mathrm{ppm}$.

This analysis strongly suggests that minimal hydrogen contamination would cause severe decarburization. Stein et al. (53) employed a similar technique to decarburize iron single crystals using zirconium at $800^{\circ} \mathrm{C}$ in a hydrogen atmosphere. The carbon content of the iron was decreased from 36 to $0.005 \mathrm{ppm}$. In a dynamic vacuum most of the hydrogen gas liberated by the vacuum chamber walls or existing as impurities in the atmosphere is pumped out and very little hydrogen is available to decarburize the system. Vacuum runs are however limited by metal vaporization at higher temperatures. One run was made with the sample enveloped in a quartz sleeve whose ends were tappered to minimize the loss of metal vapors to the cold walls of the chamber. The run made in vacuum was at $1100^{\circ} \mathrm{C}$. After 3 hours the quartz sleeve was completely fogged, and the run was terminated due to an inability to monitor the temperature of the sample any further by the optical 
pyrometer. The fogged tube was tested for metallic presence by holding a magnet over it. It was clearly magnetic. Both $\mathrm{Fe}$ and $\mathrm{Ni}$ are magnetic at room temperature, but the vapor pressure data at $1100^{\circ} \mathrm{C}$ indicates that the vapor pressure of iron is high enough that vaporization would occur in a system at $10^{-7}$ torr. We, therefore; had to solve the hydrogen problem in some other way in order to get good higher temperature data.

The tantalum adapters were replaced with oxygen-free high conductivity (OFHC) copper adapters and the zirconium getter wires with wider $\mathrm{Zr}$ strips to increase the getter surface. Gettering was done at $1400^{\circ} \mathrm{C}$ for 50 hours to remove nitrogen and oxygen from the inert gas. Lower temperature gettering at $\sim 300-400^{\circ} \mathrm{C}$ was then done for at least 12 hours to remove the traces of hydrogen. After a11 these precautions had been taken, good high temperature electrotransport data with no measurable carbon losses were obtained. However, after prolonged heating at high temperatures in a purified argon atmosphere, as in the thermotransport experiments, a loss of a few percent carbon was noter. This was overcome by lowering the maximum temperature to limit the vaporization of iron and going back to vacuum heating conditions. This seemed to alleviate the problem.

This decarburization problem has been of considerable concern to us, not only because of the time spent in pursuing it, but also because other investigators $(28,34,36)$ who have done similar studies with $\mathrm{Fe}-\mathrm{Ni}-\mathrm{C}$ and $\mathrm{Fe}-\mathrm{C}$ alloy in vacuum have not reported any problems with such decarburization. Perhaps our methods of study (non-steady state 
in electrotransport) and relatively long specimens in steady-state thermotransport are more capable of determining small carbon losses: These techniques are inherently dependent on a good mass balance in the data. 
APPENDIX B

The heats of transport $Q^{*}$ reported in this work were all obtained from plots of $\ln c$ versus $\frac{1}{T}$ as deduced from Eq. 40a. The equation is derived on the assumption of an ideal solution. In this study we used alloys with high carbon concentrations from $\sim 2000$ up to $\sim 6200 \mathrm{ppm}$ initial composition. Information available in the literature (54) indicates that these alloys are not 1deal solutions. A plot of $\ln$ a versus $\frac{1}{T}$. from Eq. 46 would therefore be more appropriate for such regular solutions (31) in determining the "true" heat of transport $Q$ * due to thermal gradient.

To determine the heat of transport $Q^{*}$ in a regular solution the heat of solution $\overline{\Delta \mathrm{H}}$ must be known, or determined independently. The free energy of formation of the solution is

$$
\overline{\Delta G}=R T \cdot \ln a_{C} \cdot
$$

Taking the partial derivative of $\overline{\Delta G} / T$ with respect to composition at constant pressure and temperature we write

$$
\left(\frac{d \overline{\Delta G} / T}{d X_{C}}\right)_{P, T}=\left(\frac{\partial \overline{\Delta G} / T}{\partial X_{C}}\right) \frac{d X_{C}}{d X_{C}}+\left(\frac{\partial \overline{\Delta G} / T}{\partial 1 / T}\right) \frac{d 1 / T}{d X_{C}}
$$

where $X_{C}=$ mole fraction, $P=$ pressure, $T=$ temperature and $\frac{\partial \overline{\Delta G} / T}{\partial 1 / T}$ is the heat of solution $\overline{\Delta H}$.

$$
\text { At constant temperature }\left(\frac{\partial \overline{\Delta G} / T}{\partial X_{C}}\right)_{P, T}=\frac{1}{T}\left(\frac{\partial \overline{\Delta G}}{\partial X_{C}}\right)_{P, T}
$$




$$
\begin{aligned}
& \left(\frac{\partial \overline{\Delta G}_{C}}{\partial X_{C}}\right)_{P, T}=R T \frac{\partial \ln a_{C}}{\partial X_{C}} \\
& \left(\frac{\partial \Delta G / T}{\partial X_{C}}\right)_{P, T}=R \frac{\partial \ln { }^{a} C}{\partial X_{C}}=
\end{aligned}
$$

The first term on the left of Eq. $57\left(\frac{d \overline{\Delta G} / T}{d X_{C}}\right)$ is obtained from a plot of $\overline{\Delta G} / T$ versus $X_{C}$ (Fig. 31). The mean value of the slopes taken from the temperatures of interest, 1073 and $1273^{\circ} \mathrm{K}$, gives a value for $\left(\frac{d \overrightarrow{\Delta G} / T}{d X_{C}}\right)_{P, T}$ of $-51.45 \mathrm{cal} / \mathrm{mol}-\mathrm{K}$. The $\overline{\Delta G} / \mathrm{T}$ versus $\mathrm{X}_{\mathrm{C}} \mathrm{plot}$ was made for the literature data of the carbon solubility limit in the Fe-Ni-C system.

Natesan and Kassner (54) deduced an equation to calculate the activity of carbon in the Fe-Ni-C system for any carbon and nickel concentration and temperature,

$$
\begin{aligned}
\ln { }_{a_{C}}(F e-N i-C)= & \ln \left(\frac{X_{C}}{1-X_{C}}\right)+\left(11.92-\frac{6330}{T}\right)\left(\frac{x_{C}}{1-X_{C}}\right)-1.845+\frac{5100}{T} \\
& -\left(2.2-\frac{7600}{T}\right) X_{N i}
\end{aligned}
$$

where $X_{C}$ and $X_{N i}$ are mole fractions of carbon and nickel respectively. By differentiating Eq. 59 with respect to $X_{C}$, a value is obtained for Eq. 58. Theoretically $\overline{\Delta H}$ is independent of temperature. But from the solubility data (Fig. 4) the carbon composition, and hence $\mathrm{X}_{\mathrm{C}}$, change with temperature. Therefore the mean value, $61.87 \mathrm{cal} / \mathrm{mol}-\mathrm{K}$, of the partial derivative of $\ln$ a as a function of $x_{C}$ taken at two different 


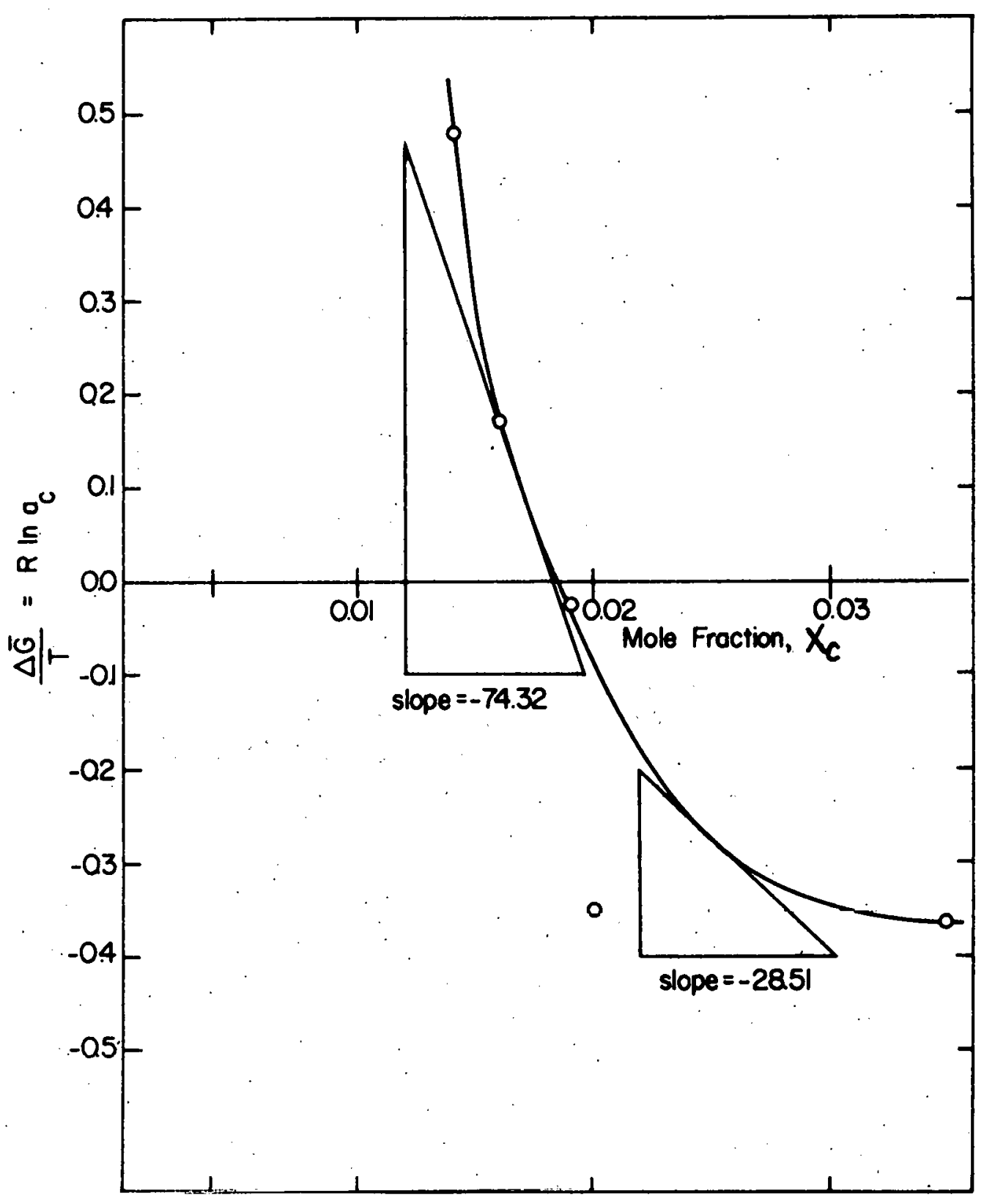

Figure 31. Plot of $\Delta \bar{G} / T$ versus $X_{c}$ carbon mole fraction. 
temperatures was used.

Rewriting Eq. 57 we obtain

$$
\begin{aligned}
\left(\frac{\partial \Delta G / T}{\partial 1 / T}\right) \frac{d 1 / T}{d X_{C}}=\frac{d}{\Delta H} \frac{1 / T}{d X_{C}}=\left[\left(\frac{d \overline{\Delta G} / T}{d X_{C}}\right)_{P, T}-\frac{\partial \Delta G / T}{\partial X_{C}}\right] \\
\overline{\Delta H}=\left[\left(\frac{d \overline{\Delta G} / T}{d X_{C}}\right)_{P, T}-\frac{\partial \Delta G / T}{\partial X_{C}}\right] \cdot \frac{d_{C}}{d 1 / T} .
\end{aligned}
$$

A plot of $X_{C}$ (carbon mole fraction) as a function of $\frac{1}{T}$ gives a slope which is equal to $\frac{\mathrm{dX}_{C}}{\mathrm{~d} / \mathrm{T}}$ needed in Eq. 61. Such a plot is shown in Fig. 32. The point outside that curve seems erratic. Similar divergent behavior was obtained for the same point in the activity calculation. Perhaps this is indicative of an anomalous behavior of the activity of carbon in the Fe-Ni-C system. An actual explanation is not attempted here. By taking the slopes of the curve from two distant points corresponding to the same temperatures used in deducing the $\ln$ a in Eq. 58, a mean value for $\frac{\mathrm{dX}_{\mathrm{C}}}{\mathrm{d} 1 / \mathrm{T}}$ was obtained as $-158.40 \mathrm{~K}$. Inserting the numerical values obtained from the plots (Figs. 31 and 32) and from the calculation using Eq. 58 into Eq. 61, we obtain

$$
\begin{aligned}
& \overline{\Delta H}=(-51.45-61.87) \mathrm{cal} / \mathrm{mol}-\mathrm{K}(-158.40) \mathrm{K} \\
& \overline{\Delta H}=17.95 \mathrm{kcal} / \mathrm{mol} .
\end{aligned}
$$

A plot of $\ln a_{C}$ versus $\frac{1}{T}$ for the 0.2 wt $\% C$ alloy ts shown in Fig. 33 . The slope of that curve gives $Q^{*}$ thermal $+\overline{\Delta \mathrm{H}}=9.74 \mathrm{kcal} / \mathrm{mol}$. Combining this with Eq. 62 we obtain 


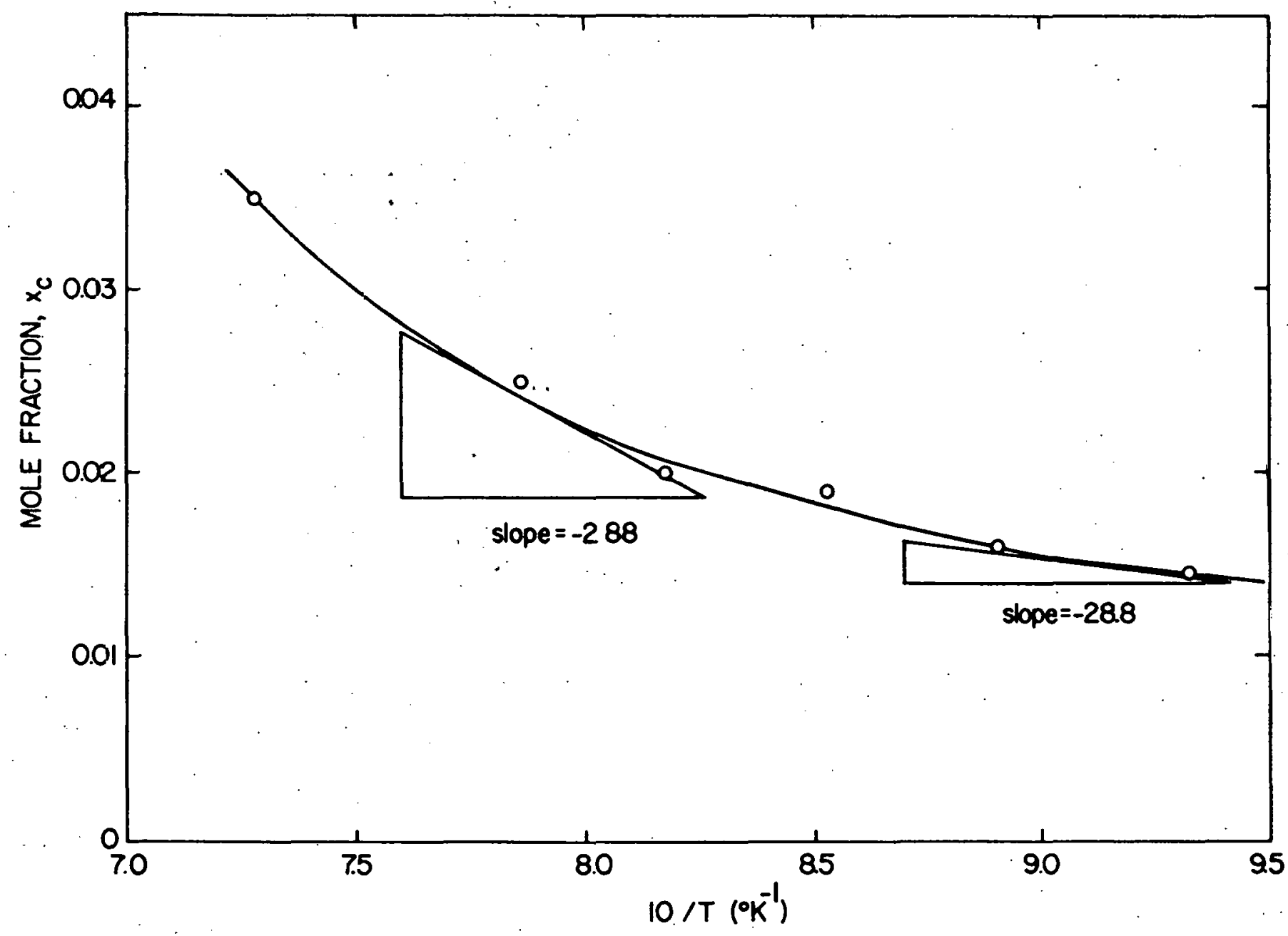

$\infty$

Figure 32: Plot of carbon mole fraction $X_{c}$ as a function of $\frac{1}{T}$. 


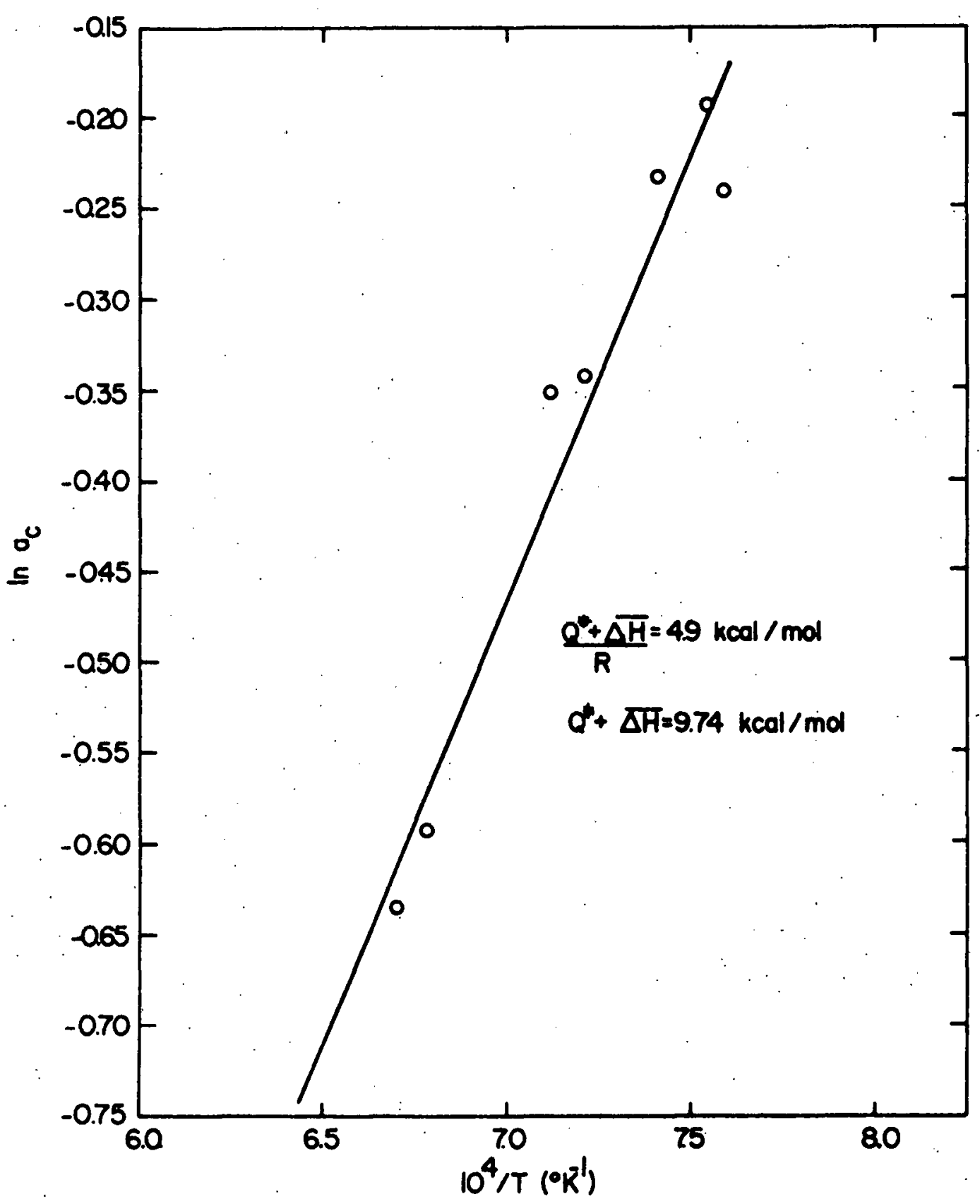

Figure 33. Least squares plot of $\ln a_{c}$ as a function of $\frac{1}{T}$ for the $0.22 \mathrm{w} \% \mathrm{C}$ alloy. 


$$
Q_{\text {thermal }}^{*}+17.95 \mathrm{kcal} / \mathrm{mol}=9.74 \mathrm{kcal} / \mathrm{mol}
$$

and

$$
\mathrm{Q}_{\text {thermal }}^{*}=-8.2 \mathrm{kcal} / \mathrm{mol} \text {. }
$$

The sign of $Q^{*}$ thermal is consistent with the observed migration to the hotter portion of the sample. The difference between this value of $Q^{*}-8.2 \mathrm{kcal} / \mathrm{mol}$ and the $-2.8 \mathrm{kcal} / \mathrm{mol}$ obtained by using ideality conditions is significant and strongly suggests the need to allow for non-ideality when working with regular solid solutions. 